\title{
Unitary representations of super Lie groups and applications to the classification and multiplet structure of super particles
}

\author{
C. Carmeli; G. Cassinelli $†$ A. Toigo $\ddagger$ V.S. Varadarajan ${ }^{\S}$
}

April 21, 2022

\begin{abstract}
It is well known that the category of super Lie groups (SLG) is equivalent to the category of super Harish-Chandra pairs (SHCP). Using this equivalence, we define the category of unitary representations (UR's) of a super Lie group. We give an extension of the classical inducing construction and Mackey imprimitivity theorem to this setting. We use our results to classify the irreducible unitary representations of semidirect products of super translation groups by classical Lie groups, in particular of the super Poincaré groups in arbitrary dimension. Finally we compare our results with those in the physical literature on the structure and classification of super multiplets.
\end{abstract}

\section{Introduction}

The classification of free relativistic super particles in SUSY quantum mechanics is well known (see for example [SS74, [FSZ81]). It is based on the technique of little (super)groups which, in the classical context, goes back to Wigner and Mackey. However the treatments of this question in the

${ }^{*}$ C. Carmeli, Dipartimento di Fisica, Università di Genova, and I.N.F.N., Sezione di Genova, Via Dodecaneso 33, 16146 Genova, Italy. e-mail: carmeli@ge.infn.it

${ }^{\dagger}$ G. Cassinelli, Dipartimento di Fisica, Università di Genova, and I.N.F.N., Sezione di Genova, Via Dodecaneso 33, 16146 Genova, Italy. e-mail: cassinelli@ge.infn.it

$\ddagger$ A. Toigo, Dipartimento di Fisica, Università di Genova, and I.N.F.N., Sezione di Genova, Via Dodecaneso 33, 16146 Genova, Italy. e-mail: toigo@ge.infn.it

$\S$ V.S.Varadarajan, Department of Mathematics, University of California at Los Angeles, Box 951555 Los Angeles, CA 90095-1555, USA. e-mail: vsv@math.ucla.edu 
physics literature make the implicit assumption that the technique of little groups remains valid in the SUSY set up without any changes; in particular no attempt is made in currently available treatments to exhibit the SUSY transformations explicitly for the super particle. The aim of this paper is to remedy this situation by laying a precise mathematical foundation for the theory of unitary representations of super Lie groups, and then to apply it to the case of the super Poincaré groups. In the process of doing this we clarify and extend the results in the physical literature to Minkowskian spacetimes of arbitrary dimension $D \geq 4$ and $N$-extended supersymmetry for arbitrary $N \geq 1$.

Super Lie groups differ from classical Lie groups in a fundamental way: one should think of them as group-valued functors rather than groups. As long as only finite dimensional representations are being considered, there is no difficulty in adapting the functorial theory to study representations. However, this marriage of the functorial approach with representation theory requires modifications when one considers infinite dimensional representations. Our entire approach is based on two observations. The first is to view a super Lie group as a Harish-Chandra pair, namely a pair $\left(G_{0}, \mathfrak{g}\right)$ where $G_{0}$ is a classical Lie group, $\mathfrak{g}$ is a super Lie algebra which is a $G_{0}$-module, $\operatorname{Lie}\left(G_{0}\right)=\mathfrak{g}_{0}$, and the action of $\mathfrak{g}_{0}$ on $\mathfrak{g}$ is the differential of the action of $G_{0}$. That this is a valid starting point is justified by the result DM99] that the category of super Lie groups is equivalent to the category of Harish-Chandra pairs. This point of view leads naturally to define a unitary representation of a super Lie group $\left(G_{0}, \mathfrak{g}\right)$ as a classical unitary representation of $G_{0}$ together with a compatible infinitesimal unitary action of $\mathfrak{g}$. This is in fact very close to the approach of the physicists. The source of our second observation is more technical and is the fact, which is a consequence of the commutation rules, that the operators corresponding to the odd elements of $\mathfrak{g}$ are in general unbounded and so care is needed to work with them. Our second observation is in fact a basic result of this paper, namely, that the commutation rules and the symmetry requirements that are implicit in a supersymmetric theory force the unbounded odd operators to be well behaved and lead to an essentially unique way to define a unitary representation of a super Lie group. Of course this aspect was not treated in the physical literature, not only because representations of the big super Lie groups were not considered, but even more, because only finite dimensional super representations of the little groups were considered, where unbounded phenomena obviously do not occur. Our treatment has the additional feature that it is able to handle the construction of super particles with infinite spin also.

In the first section of the paper we treat the foundations of the theory of 
unitary representations of a super Lie group based on these two observations. The basic result is Proposition 2 which asserts that the odd operators are essentially self adjoint on their domain and that the representation of the Harish-Chandra pair, extended to the space of $C^{\infty}$ vectors of the representation of $G_{0}$, is unique. This result is essential to everything we do and shows that the formal aspects of representations of super Lie groups already control all their analytic aspects. The second section discusses the imprimitivity theorem in the super context. Here an important assumption is made, namely that the super homogeneous space on which we have the system of imprimitivity is a purely even manifold, or equivalently, the sub super Lie group $\left(H_{0}, \mathfrak{h}\right)$ defining the system of imprimitivity has the same odd dimension as the ambient super Lie group, i.e., $\mathfrak{h}_{1}=\mathfrak{g}_{1}$. This restriction, although severe, is entirely adequate for treating super Poincaré groups and more generally a wide class of super semi direct products. The main result is Theorem 2 which asserts that the inducing functor is an equivalence of categories from the category of unitary representations of $\left(H_{0}, \mathfrak{h}\right)$ to the category of super systems of imprimitivity based on $G_{0} / H_{0}$. These two sections complete the foundational aspects of this paper.

The third section is concerned with applications and bringing our treatment as close as possible to the ones in the literature. We consider a super semi direct product of a super translation group with a classical Lie group $L_{0}$ acting on it. No special assumption is made about the action of $L_{0}$ on $\mathfrak{g}_{1}$, so that the class of super Lie groups considered is vastly larger than the ones treated in the physical literature, where this action is always assumed to be spinorial. If $T_{0}$ is the vector group which is the even part of the super translation group, the theory developed in $\$ \$ 3 / 4$ leads to the result that the irreducible representations of $\left(G_{0}, \mathfrak{g}\right)$ are in one-one correspondence with certain $L_{0}$-orbits in the dual $T_{0}^{*}$ of $T_{0}$ together with certain irreducible representations of the super Lie groups (little groups) which are stabilizers of the points in the orbits. This is the super version of the classical little group method (Theorem [3).

It is from this point on that the SUSY theory acquires its own distinctive flavor. In the first place, unlike the classical situation, Theorem 3 stipulates that not all orbits are allowed, only those belonging to a suitable subset $T_{0}^{+}$. We shall call these orbits admissible. These are the orbits where the little super group admits an irreducible unitary representation which restricts to a character of $T_{0}$. These representations will be called admissible. These orbits satisfy a positivity condition which we interpret as the condition of positivity of energy. This condition is therefore necessary for admissibility. However it requires some effort to show that it is also sufficient for admissibility, and then 
to determine all the irreducible unitary representations of the little super Lie group at $\lambda$ (Theorem 4).

The road to Theorem 4 is somewhat complicated. Let $\lambda \in T_{0}^{*}$ be fixed. The classical stabilizer of $\lambda$ is $T_{0} L_{0}^{\lambda}$, where $L_{0}^{\lambda}$ is the classical little group at $\lambda$, namely the stabilizer of $\lambda$ in $L_{0}$. The super stabilizer of $\lambda$ is the super Lie group $S^{\lambda}$ defined by

$$
S^{\lambda}=\left(T_{0} L_{0}^{\lambda}, \mathfrak{g}^{\lambda}\right), \quad \mathfrak{g}^{\lambda}=\mathfrak{t}_{0} \oplus \mathfrak{l}_{0}^{\lambda} \oplus \mathfrak{g}_{1} .
$$

(By convention, the Lie algebra of a Lie group is denoted by the corresponding gothic letter.) Given $\lambda$, there is an associated $L_{0}^{\lambda}$-invariant quadratic form $\Phi_{\lambda}$ on $\mathfrak{g}_{1}$ which will be nonnegative definite; the nonnegativity of $\Phi_{\lambda}$ is the positive energy condition mentioned earlier. This form need not be strictly positive, but one can pass to the quotient $\mathfrak{g}_{1 \lambda}$ of $\mathfrak{g}_{1}$ by its radical, and obtain a positive definite quadratic vector space on which the classical part $L_{0}^{\lambda}$ of the little group operates. So we obtain a map

$$
j_{\lambda}: L_{0}^{\lambda} \longrightarrow \mathrm{O}\left(\mathfrak{g}_{1 \lambda}\right)
$$

Now $L_{0}^{\lambda}$ need not be connected and so $j_{\lambda}$ need not map into $\operatorname{SO}\left(\mathfrak{g}_{1 \lambda}\right)$. Also, since we are not making any assumption about the action of $L_{0}$ on $\mathfrak{g}_{1}$, it is quite possible that $\operatorname{dim}\left(\mathfrak{g}_{1 \lambda}\right)$ could be odd. We introduce the subgroup

$$
L_{00}^{\lambda}= \begin{cases}j_{\lambda}^{-1}\left(\mathrm{SO}\left(\mathfrak{g}_{1 \lambda}\right)\right) & \text { if } j_{\lambda}\left(L_{0}^{\lambda}\right) \not \subset \mathrm{SO}\left(\mathfrak{g}_{1 \lambda}\right) \text { and } \operatorname{dim}\left(\mathfrak{g}_{1 \lambda}\right) \text { is even } \\ L_{0}^{\lambda} & \text { otherwise. }\end{cases}
$$

Then $L_{00}^{\lambda}$ is either the whole of $L_{0}^{\lambda}$ or a (normal) subgroup of index 2 in it. Theorem 4 asserts that there is a functorial map

$$
r \longmapsto \theta_{r \lambda}
$$

which is an equivalence of categories from the category of unitary projective representations $r$ of $L_{00}^{\lambda}$ corresponding to a certain canonical multiplier $\mu_{\lambda}$, to the category of admissible unitary representations of the super group $S^{\lambda}$. In particular, the admissible irreducible unitary representations of $S^{\lambda}$ correspond one-one to irreducible unitary $\mu_{\lambda}$-representations of $L_{00}^{\lambda}$. We shall now explain how this correspondence is set up.

The odd operators of the super representation of the little group are obtained from a self adjoint representation of the Clifford algebra of $\mathfrak{g}_{1 \lambda}$. Since any such is a multiple of an essentially unique one, we begin with an irreducible self adjoint representation. Let us call it $\tau_{\lambda}$. In the space of $\tau_{\lambda}$ 
one can define in an essentially unique manner a projective representation $\kappa_{\lambda}$ of $L_{0}^{\lambda}$ that intertwines $\tau_{\lambda}$ and its transforms by elements of $L_{0}^{\lambda}$ :

$$
\kappa_{\lambda}(t) \tau_{\lambda}(X) \kappa_{\lambda}(t)^{-1}=\tau_{\lambda}(t X)
$$

The class of the multiplier $\mu_{\lambda}$ in $H^{2}\left(L_{0}^{\lambda}, \mathbb{T}\right)$ is then uniquely determined. We can normalize $\mu_{\lambda}$ so that it takes only \pm 1 -values. Starting from any unitary $\mu_{\lambda}$-representation $r$ of $L_{00}^{\lambda}$ we build the $\mu_{\lambda}$-representation

$$
\operatorname{Ind}(r)
$$

of $L_{0}^{\lambda}$ induced by $r$. Then the admissible unitary representation $\theta_{r \lambda}$ of $S^{\lambda}$ corresponding to $r$ is given by

$$
\theta_{r \lambda}=\left(e^{i \lambda(t)} \operatorname{Ind}(r) \otimes \kappa_{\lambda}, 1 \otimes \tau_{\lambda}\right)
$$

the fact that $\mu_{\lambda}$ is \pm 1 -valued implies that $\theta_{r \lambda}$ is an ordinary rather than a projective representation. The representation $\Theta_{r \lambda}$ of the super Lie group $\left(G_{0}, \mathfrak{g}\right)$ induced by $\theta_{r \lambda}$ from $S^{\lambda}$ is then irreducible if $r$ is irreducible. Theorem 5 asserts that all the irreducible unitary representations of the super Lie group are parametrized bijectively as above by the irreducible representations of $L_{00}^{\lambda}$, thus giving the super version of the classical theory.

The representation $\kappa_{\lambda}$ is therefore at the heart of the theory of irreducible unitary representations of super semi direct products. It is finite dimensional; in fact it is the lift via $j_{\lambda}$ to $L_{0}^{\lambda}$ of the spin representation of the quadratic vector space. It is dependent only on $\lambda$. Clearly, the representation $\operatorname{Ind}(r) \otimes \kappa_{\lambda}$ of $L_{0}^{\lambda}$ will not in general be irreducible even if $r$ is. The irreducible constituents of Ind $(r) \otimes \kappa_{\lambda}$ then define, via the classical procedure of inducing, irreducible unitary representations of $G_{0}$ which are the constituents of the even part of the full super representation. This family of irreducible representations of $G_{0}$ is the multiplet of the irreducible representation of the super semi direct product in question. If $\mu_{\lambda}$ is trivial, we can choose $r$ to be trivial, and the corresponding multiplet is the fundamental multiplet. Thus $\kappa_{\lambda}$ determines the entire correspondence in a simple manner.

In 4.3 we discuss the case of the super Poincaré groups. We consider spacetimes of Minkowski signature and of arbitrary dimension $D \geq 4$ together with $N$-extended supersymmetry for arbitrary $N \geq 1$. In this case, the groups $L_{0}^{\lambda}$ are all connected and $L_{0}^{\lambda}=L_{00}^{\lambda}$. Moreover, the multiplier $\mu_{\lambda}$ becomes trivial, so that $\kappa_{\lambda}$ becomes an ordinary representation (Lemma 13). Hence

$$
\theta_{r \lambda}=\left(e^{i \lambda(t)} r \otimes \kappa_{\lambda}, 1 \otimes \tau_{\lambda}\right)
$$


Thus in this case we finally reach the conclusion that the super particles are parametrized by the admissible orbits and irreducible unitary representations of the stabilizers of the classical little groups, exactly as in the classical theory. The positive energy condition $\Phi_{\lambda} \geq 0$ becomes just that $\lambda$, which we replace by $p$ to display the fact that it is a momentum vector, lies in the closure of the forward light cone. Thus the orbits of imaginary mass are excluded by supersymmetry (Theorem [6). Our approach enables us to handle super particles with infinite spin in the same manner as those with finite spin because of the result that the odd operators of the little group are bounded (Lemma 10).

Finally, in 4.4 we give the explicit determination of $\kappa_{p}$ when the spacetime has arbitrary dimension $D \geq 4$ and we have $N$-extended supersymmetry. The results in the physical literature are in general only for $D=4$.

The literature on supersymmetric representation theory is very extensive. We have been particularly influenced by [SS74, [Fer01, [Fer03, [FSZ81]. In [DP85, [DP86], DP87] Dobrev et al. discuss representations of superconformal groups induced from a maximal parabolic subgroup. The papers [SS74] gave the structure of $\kappa_{p}$ for $N=1$ supersymmetry, while [FSZ81] gave a very complete treatment of the structure of $\kappa_{p}$ in the case of extended supersymmetry when $D=4$, including the case of central charges.

\section{Super Lie groups and their unitary repre- sentations}

\subsection{Super Hilbert spaces}

All sesquilinear forms are linear in the first argument and conjugate linear in the second. We use the usual terminology of super geometry as in [DM99, Var04]. A super Hilbert space (SHS) is a super vector space $\mathcal{H}=\mathcal{H}_{0} \oplus \mathcal{H}_{1}$ over $\mathbb{C}$ with a scalar product $(\cdot, \cdot)$ such that $\mathcal{H}$ is a Hilbert space under $(\cdot, \cdot)$, and $\mathcal{H}_{i}(i=0,1)$ are mutually orthogonal closed linear subspaces. If we define

$$
\langle x, y\rangle= \begin{cases}0 & \text { if } x \text { and } y \text { are of opposite parity } \\ (x, y) & \text { if } x \text { and } y \text { are even } \\ i(x, y) & \text { if } x \text { and } y \text { are odd }\end{cases}
$$

then $\langle x, y\rangle$ is an even super Hermitian form with

$$
\langle y, x\rangle=(-1)^{p(x) p(y)} \overline{\langle x, y\rangle},\langle x, x\rangle>0(x \neq 0 \text { even }), i^{-1}\langle x, x\rangle>0(x \neq 0 \text { odd }) .
$$


If $T(\mathcal{H} \rightarrow \mathcal{H})$ is a bounded linear operator, we denote by $T^{*}$ its Hilbert space adjoint and by $T^{\dagger}$ its super adjoint given by $\langle T x, y\rangle=(-1)^{p(T) p(x)}\left\langle x, T^{\dagger} y\right\rangle$. Clearly $T^{\dagger}$ is bounded, $p(T)=p\left(T^{\dagger}\right)$, and $T^{\dagger}=T^{*}$ or $-i T^{*}$ according as $T$ is even or odd. For unbounded $T$ we define $T^{\dagger}$ in terms of $T^{*}$ by the above formula. These definitions are equally consistent if we use $-i$ in place of $i$. But our convention is as above.

\subsection{SUSY quantum mechanics}

In SUSY quantum mechanics in a SHS $\mathcal{H}$, it is usual to stipulate that the Hamiltonian $H=X^{2}$ where $X$ is an odd operator Wit82; it is customary to argue that this implies that $H \geq 0$ (positivity of energy); but this is true only if we know that $H$ is essentially self adjoint on the domain of $X^{2}$. We shall now prove two lemmas which clarify this situation and will play a crucial role when we consider systems with a super Lie group of symmetries.

If $A$ is a linear operator on $\mathcal{H}$, we denote by $D(A)$ its domain. We always assume that $D(A)$ is dense in $\mathcal{H}$, and then refer to it as densely defined. We write $A \prec B$ if $D(A) \subset D(B)$ and $B$ restricts to $A$ on $D(A) ; A$ is symmetric iff $A \prec A^{*}$, and then $A$ has a closure $\bar{A}$. $A \prec B \Longrightarrow B^{*} \prec A^{*}$. If $A$ is symmetric we say that it is essentially self adjoint if $\bar{A}$ is self adjoint; in this case $A^{*}=\bar{A}$. If $A$ is symmetric and $B$ is a symmetric extension of $A$, then $A \prec B \prec A^{*}$; in fact $A \prec A^{*}$ and $A \prec B \prec B^{*}$, and so $B^{*} \prec A^{*}$ and $A \prec B \prec B^{*} \prec A^{*}$. If $A$ is self adjoint and $L \subset D(A)$, we say that $L$ is a core for $A$ if $A$ is the closure of its restriction to $L$. A vector $\psi \in \mathcal{H}$ is analytic for a symmetric operator $H$ if $\psi \in D\left(H^{n}\right)$ for all $n$ and the series $\sum_{n} t^{n}(n !)^{-1}\left\|H^{n} \psi\right\|<\infty$ for some $t>0$. It is a well known result of Nelson Nel59 that if $D \subset D(H)$ and contains a dense set of analytic vectors, then $H$ is essentially self adjoint on $D$. In this case $\psi \in D(H)$ is analytic for $H$ if and only if $t \longmapsto e^{i t H} \psi$ is analytic in $t \in \mathbb{R}$. If $A$ is self adjoint, then $A^{2}$, defined on the domain $D\left(A^{2}\right)=\{\psi \mid \psi, A \psi \in D(A)\}$, is self adjoint; this is well known and follows easily from the spectral theorem.

Lemma 1 Let $H$ be a self adjoint operator on $\mathcal{H}$ and $U(t)=e^{i t H}$ the corresponding one parameter unitary group. Let $\mathcal{B} \subset D(H)$ be a dense $U$-invariant linear subspace. We then have the following.

(i) $\mathcal{B}$ is a core for $H$.

(ii) Let $X$ be a symmetric operator with $\mathcal{B} \subset D(X)$ such that $X \mathcal{B} \subset D(X)$ and $\left.X^{2}\right|_{\mathcal{B}}=\left.H\right|_{\mathcal{B}}$. Then $\left.X\right|_{\mathcal{B}}$ is essentially self adjoint, $\overline{\left.X\right|_{\mathcal{B}}}=\bar{X}$ and $\bar{X}^{2}=H$. 
In particular, $H \geq 0, D(H) \subset D(\bar{X})$. Finally, these results are valid if we only assume that $\mathcal{B}$ is invariant under $H$ and contains a dense set of analytic vectors.

Proof. Let $H_{1}=\left.H\right|_{\mathcal{B}}$. We must show that if $L(\lambda)(\lambda \in \mathbb{C})$ is the subspace of $\psi$ such that $H_{1}^{*} \psi=\lambda \psi$, then $L(\lambda)=0$ if $\Im(\lambda) \neq 0$. Now $L(\lambda)$ is a closed subspace. Moreover, as $H_{1}$ is invariant under $U$, so is $H_{1}^{*}$ and so $L(\lambda)$ is invariant under $U$ also. So the vectors in $L(\lambda)$ that are $C^{\infty}$ for $U$ are dense in $L(\lambda)$ and so it is enough to prove that 0 is the only $C^{\infty}$ vector in $L(\lambda)$. But $H=H^{*} \prec H_{1}^{*}$ while the $C^{\infty}$ vectors for $U$ are all in $D(H)$, and so if $\psi$ is a $C^{\infty}$ vector in $L(\lambda), H \psi=H_{1}^{*} \psi=\lambda \psi$. This is a contradiction since $H$ is self adjoint and so all its eigenvalues are real. This proves (i).

Let $X_{1}=\left.X\right|_{\mathcal{B}}$. Clearly, $X_{1}$ is symmetric on $\mathcal{B}$. It is enough to show that $X_{1}$ is essentially self adjoint and ${\overline{X_{1}}}^{2}=H$, since in this case $X_{1} \prec X \prec X_{1}^{*}=$ $\overline{X_{1}}$ and hence $\bar{X}=\overline{X_{1}}$. We have $X_{1}^{2}=H_{1}$. So $H \geq 0$ on $\mathcal{B}$ and hence $H \geq 0$ by (i). Again it is a question of showing that for $\lambda \in \mathbb{C}$ with $\Im(\lambda) \neq 0$, we must have $M(\lambda)=0$ where $M(\lambda)$ is the eigenspace for $X_{1}^{*}$ for the eigenvalue $\lambda$. Let $\psi \in M(\lambda)$. Now, for $\varphi \in \mathcal{B}$,

$$
\left(X_{1}^{2} \varphi, \psi\right)=\left(X_{1} \varphi, X_{1}^{*} \psi\right)=\bar{\lambda}\left(X_{1} \varphi, \psi\right)=\bar{\lambda}^{2}(\varphi, \psi)=\left(\varphi, \lambda^{2} \psi\right) .
$$

Hence $\psi \in D\left(\left(X_{1}^{2}\right)^{*}\right)$ and $\left(X_{1}^{2}\right)^{*} \psi=\lambda^{2} \psi$. But $X_{1}^{2}=H_{1}$ and so $\left(X_{1}^{2}\right)^{*}=H_{1}^{*}=$ $H$ by (i). So $H \psi=\lambda^{2} \psi$. Hence $\lambda^{2}$ is real and $\geq 0$. This contradicts the fact that $\Im(\lambda) \neq 0$. Furthermore, $X_{1}^{2} \prec{\overline{X_{1}}}^{2}$ and so ${\overline{X_{1}}}^{2}=\left({\overline{X_{1}}}^{2}\right)^{*} \prec\left(X_{1}^{2}\right)^{*}=$ $H_{1}^{*}=H$. On the other hand, as ${\overline{X_{1}}}^{2}$ is closed, $H=\bar{H}_{1}=\overline{X_{1}^{2}} \prec{\overline{X_{1}}}^{2}$. So $H=\bar{X}_{1}^{2}=\bar{X}^{2}$. This means that $D(H) \subset D(\bar{X})$.

Finally, let us assume that $H \mathcal{B} \subset \mathcal{B}$ and that $\mathcal{B}$ contains a dense set of analytic vectors for $H$. Clearly $\mathcal{B}$ is a core for $H$. If $\psi \in \mathcal{B}$ is analytic for $H$ we have $X^{2 n} \psi=H^{n} \psi \in \mathcal{B}$ and $X^{2 n+1} \psi \in D(X)$ by assumption, and

$$
\left\|X^{n} \psi\right\|^{2}=\left|\left(H^{n} \psi, \psi\right)\right| \leq\|\psi\|\left\|\mid H^{n} \psi\right\| \leq M^{n} n !
$$

for some $M>0$ and all $n$. Thus $\psi$ is analytic for $X$ and so its essential self adjointness is a consequence of the theorem of Nelson. The rest of the argument is unchanged.

Lemma 2 Let $A$ be a self adjoint operator in $\mathcal{H}$. Let $M$ be a smooth (resp. analytic) manifold and $f(M \longrightarrow \mathcal{H})$ a smooth (resp. analytic) map. We assume that (i) $f(M) \subset D\left(A^{2}\right)$ and (ii) $A^{2} f: m \longmapsto A^{2} f(m)$ is a smooth (resp. analytic) map of $M$ into $\mathcal{H}$. Then $A f: m \longmapsto A f(m)$ is a smooth 
(resp. analytic) map of $M$ into $\mathcal{H}$. Moreover, if $E$ is any smooth differential operator on $M,(E f)(m) \in D\left(A^{2}\right)$ for all $m \in M$, and

$$
E\left(A^{2} f\right)=A^{2} E f, \quad E(A f)=A E f .
$$

Proof. It is standard that if $g(M \longrightarrow \mathcal{H})$ is smooth (resp. analytic) and $L$ is a bounded linear operator on $\mathcal{H}$, then $L g$ is a smooth (resp. analytic) map. We have

$$
A \psi=A\left(I+A^{2}\right)^{-1}\left(I+A^{2}\right) \psi, \quad \psi \in D\left(A^{2}\right) .
$$

Moreover $A\left(I+A^{2}\right)^{-1}$ is a bounded operator. Now $\left(I+A^{2}\right) f$ is smooth (resp. analytic) and so it is immediate from the above that $A f$ is smooth (resp. analytic).

For the last part we assume that $M$ is an open set in $\mathbb{R}^{n}$ since the result is clearly local. Let $x^{i}(1 \leq i \leq n)$ be the coordinates and let $\partial_{j}=\partial / \partial x^{j}, \partial^{\alpha}=$ $\partial_{1}^{\alpha_{1}} \ldots \partial_{n}^{\alpha_{n}}$ where $\alpha=\left(\alpha_{1}, \ldots, \alpha_{n}\right)$. It is enough to prove that

$$
\left(\partial^{\alpha} f\right)(m) \in D\left(A^{2}\right), \quad A^{2} \partial^{\alpha} f=\partial^{\alpha}\left(A^{2} f\right), \quad A \partial^{\alpha} f=\partial^{\alpha}(A f) .
$$

We begin with a simple observation. Since $\left(A^{2} \psi, \psi\right)=\|A \psi\|^{2}$ for all $\psi \in D\left(A^{2}\right)$, it follows that whenever $\psi_{n} \in D\left(A^{2}\right)$ and $\left(\psi_{n}\right)$ and $\left(A^{2} \psi_{n}\right)$ are Cauchy sequences, then $\left(A \psi_{n}\right)$ is also a Cauchy sequence; moreover, if $\psi=\lim _{n} \psi_{n}$, then $\psi \in D\left(A^{2}\right)$ and $A^{2} \psi=\lim _{n} A^{2} \psi_{n}, A \psi=\lim _{n} A \psi_{n}$. This said, we shall prove the above formulae by induction on $|\alpha|=\alpha_{1}+\cdots+\alpha_{n}$. Assume them for $|\alpha| \leq r$ and fix $j, 1 \leq j \leq n$. Let $g=\partial^{\alpha} f,|\alpha|=r$. Let

$g_{h}(x)=\frac{1}{h}\left(g\left(x^{1}, \ldots, x^{j}+h, \ldots, x^{n}\right)-g\left(x^{1}, \ldots, x^{n}\right)\right) \quad\left(h\right.$ is in $j^{\text {th }}$ place $)$.

Then, as $h \rightarrow 0, g_{h}(x) \rightarrow \partial_{j} \partial^{\alpha} f(x)$ while $A^{2} g_{h}(x)=\left(\partial^{\alpha} A^{2} f\right)_{h}(x) \rightarrow$ $\partial_{j} \partial^{\alpha} A^{2} f(x)$, and $A g_{h}(x)=\left(\partial^{\alpha} A f\right)_{h}(x) \rightarrow \partial_{j} \partial^{\alpha} A f(x)$. From the observation made above we have $\partial_{j} \partial^{\alpha} f(x) \in D\left(A^{2}\right)$ and $A^{2}$ and $A$ map it respectively into $\partial_{j} \partial^{\alpha} A^{2} f(x)$ and $\partial_{j} \partial^{\alpha} A f(x)$.

Definition 1 For self adjoint operators $L, X$ with $L$ bounded, we write $L \leftrightarrow$ $X$ to mean that $L$ commutes with the spectral projections of $X$.

Lemma 3 Let $X$ be a self adjoint operator and $\mathcal{B}$ a dense subspace of $\mathcal{H}$ which is a core for $X$ such that $X \mathcal{B} \subset \mathcal{B}$. If $L$ is a bounded self adjoint operator such that $L \mathcal{B} \subset \mathcal{B}$, then the following are equivalent: $(i) L X=X L$ on $\mathcal{B}($ ii $) L \bar{X}=\bar{X} L$ on $D(\bar{X})$ (this carries with it the inclusion $L D(\bar{X}) \subset$ $D(\bar{X})$ ) (iii) $L \leftrightarrow \bar{X}$. In this case $e^{i t L} \bar{X}=\bar{X} e^{i t L}$ for all $t \in \mathbb{R}$. 
Proof. (i) $\Longleftrightarrow$ (ii). Let $b \in D(\bar{X})$. Then there is a sequence $\left(b_{n}\right)$ in $\mathcal{B}$ such that $b_{n} \rightarrow b, X b_{n} \rightarrow \bar{X} b$. Then $X L b_{n}=L X b_{n} \rightarrow L \bar{X} b$. Since $L b_{n} \rightarrow L b$ we infer that $L b \in D(\bar{X})$ and $\bar{X} L b=L \bar{X} b$. This proves (i) $\Longrightarrow$ (ii). The reverse implication is trivial.

(ii) $\Longrightarrow$ (iii). We have $L^{n} \bar{X} b=\bar{X} L^{n} b$ for all $b \in D(\bar{X}), n \geq 1$. So $e^{i t L} \bar{X} b=$ $\sum_{n}\left((i t)^{n} / n !\right) \bar{X} L^{n} b$. If $s_{N}=\sum_{n \leq N}\left((i t)^{n} / n !\right) L^{n} b$, then $s_{N} \rightarrow e^{i t L} b, \bar{X} s_{N} \rightarrow$ $e^{i t L} \bar{X} b$. So $e^{i t L} b \in D(\bar{X})$ and $\bar{X} e^{i t L} b=e^{i t L} \bar{X} b$. If $U(t)=e^{i t L}$, this means that $U(t) \bar{X} U(t)^{-1}=\bar{X}$ and so, by the uniqueness of the spectral resolution of $X, U(t)$ commutes with the spectral projections of $\bar{X}$. But then $L \leftrightarrow \bar{X}$. (iii) $\Longrightarrow$ (i). Under (iii) we have $U(t) \bar{X} U(t)^{-1}=\bar{X}$ or $\bar{X} U(t) b=U(t) \bar{X} b$ for $b \in D(\bar{X}), U(t) b$ being in $D(\bar{X})$ for all $t$. Let $b_{t}=(i t)^{-1}(U(t) b-b)$. Then $\bar{X} b_{t}=(i t)^{-1}(U(t) \bar{X} b-\bar{X} b)$. Hence, as $t \rightarrow 0, b_{t} \rightarrow L b$ while $\bar{X} b_{t} \rightarrow L \bar{X} b$. Hence $L b \in D(\bar{X})$ and $\bar{X} L b=L \bar{X} b$. Thus we have (ii), hence (i).

\subsection{Unitary representations of super Lie groups}

We take the point of view DM99 that a super Lie group (SLG) is a super Harish-Chandra pair $\left(G_{0}, \mathfrak{g}\right)$ that is a pair $\left(G_{0}, \mathfrak{g}\right)$ where $G_{0}$ is a classical Lie group, $\mathfrak{g}$ is a super Lie algebra which is a $G_{0}$-module, $\operatorname{Lie}\left(G_{0}\right)=\mathfrak{g}_{0}$, and the action of $\mathfrak{g}_{0}$ on $\mathfrak{g}$ is the differential of the action of $G_{0}$. The notion of morphisms between two super Lie groups in the above sense is the obvious one from which it is easy to see what is meant by a finite dimensional representation of a $\operatorname{SLG}\left(G_{0}, \mathfrak{g}\right)$ : it is a triple $\left(\pi_{0}, \pi, V\right)$ where $\pi_{0}$ is an even representation of $G_{0}$ in a super vector space $V$ of finite dimension over $\mathbb{C}$, i.e., a representation such that $\pi_{0}(g)$ is even for all $g \in G_{0} ; \pi$ is a representation of the super Lie algebra $\mathfrak{g}$ in $V$ such that $\left.\pi\right|_{\mathfrak{g}_{0}}=d \pi_{0}$; and

$$
\pi(g X)=\pi_{0}(g) \pi(X) \pi_{0}(g)^{-1}, \quad g \in G_{0}, X \in \mathfrak{g}_{1} .
$$

If $V$ is a SHS and $\pi(X)^{\dagger}=-\pi(X)$ for all $X \in \mathfrak{g}$, we say that $\left(\pi_{0}, \pi, V\right)$ is a unitary representation (UR) of the SLG $\left(G_{0}, \mathfrak{g}\right)$. The condition on $\pi$ is equivalent to saying that $\pi_{0}$ is a unitary representation of $G_{0}$ in the usual sense and $\pi(X)^{*}=-i \pi(X)$ for all $X \in \mathfrak{g}_{1}$. It is then clear that a finite dimensional UR of $\left(G_{0}, \mathfrak{g}\right)$ is a triple $\left(\pi_{0}, \pi, V\right)$, where

(a) $\pi_{0}$ is an even unitary representation of $G_{0}$ is a SHS $V$;

(b) $\pi$ is a linear map of $\mathfrak{g}_{1}$ into the space $\mathfrak{g l}(V)_{1}$ of odd endomorphisms of $V$ with $\pi(X)^{*}=-i \pi(X)$ for all $X \in \mathfrak{g}_{1}$;

(c) $d \pi_{0}([X, Y])=\pi(X) \pi(Y)+\pi(Y) \pi(X)$ for $X, Y \in \mathfrak{g}_{1}$; 
(d) $\pi\left(g_{0} X\right)=\pi_{0}\left(g_{0}\right) \pi(X) \pi_{0}\left(g_{0}\right)^{-1}$ for $X \in \mathfrak{g}_{1}, g_{0} \in G_{0}$.

Let

$$
\zeta=e^{-i \pi / 4}, \quad \rho(X)=\zeta \pi(X) .
$$

Then, we may replace $\pi(X)$ by $\rho(X)$ for $X \in \mathfrak{g}_{1}$; the condition (b) becomes the requirement that $\rho(X)$ is self adjoint for all $X \in \mathfrak{g}_{1}$, while the commutation rule in condition (c) becomes

$$
-i d \pi_{0}([X, Y])=\rho(X) \rho(Y)+\rho(Y) \rho(X), \quad X, Y \in \mathfrak{g}_{1} .
$$

If we want to extend this definition to the infinite dimensional context it is necessary to take into account the fact that the $\pi(X)$ for $X \in \mathfrak{g}_{1}$ will in general be unbounded; indeed, from (c) above we find that $d \pi_{0}([X, X])=$ $2 \pi(X)^{2}$, and as $d \pi_{0}$ typically takes elements of $\mathfrak{g}_{0}$ into unbounded operators, the $\pi(X)$ cannot be bounded. So the concept of a UR of a SLG in the infinite dimensional case must be formulated with greater care to take into account the domains of definition of the $\pi(X)$ for $X \in \mathfrak{g}_{1}$. In the physics literature this aspect is generally ignored. We shall prove below that contrary to what one may expect, the domain restrictions can be formulated with great freedom, and the formal and rigorous pictures are essentially the same. In particular, the concept of a UR of a super Lie group is a very stable one and allows great flexibility of handling.

If $V$ is a super vector space (not necessarily finite dimensional), we write $\operatorname{End}(V)$ for the super algebra of all endomorphisms of $V$. If $\pi_{0}$ is a unitary representation of $G_{0}$ in a Hilbert space $\mathcal{H}$, we write $C^{\infty}\left(\pi_{0}\right)$ for the subspace of differentiable vectors in $\mathcal{H}$ for $\pi_{0}$. We denote by $C^{\omega}\left(\pi_{0}\right)$ the subspace of analytic vectors of $\pi_{0}$. Here we recall that a vector $v \in \mathcal{H}$ is called a differentiable vector (resp. analytic vector) for $\pi_{0}$ if the map $g \mapsto \pi_{0}(g) v$ is smooth (resp. analytic). If $\mathcal{H}$ is a SHS and $\pi_{0}$ is even, then $C^{\infty}\left(\pi_{0}\right)$ and $C^{\omega}\left(\pi_{0}\right)$ are $\pi_{0}$-invariant dense linear super subspaces. We also need the following fact which is standard but we shall give a partial proof because the argument will be used again later.

Lemma $4 C^{\infty}\left(\pi_{0}\right)$ and $C^{\omega}\left(\pi_{0}\right)$ are stable under $d \pi_{0}\left(\mathfrak{g}_{0}\right)$. For any $Z \in \mathfrak{g}_{0}$, $i d \pi_{0}(Z)$ is essentially self adjoint both on $C^{\infty}\left(\pi_{0}\right)$ and on $C^{\omega}\left(\pi_{0}\right)$; moreover, for any $Z_{1}, \ldots, Z_{r} \in \mathfrak{g}_{0}$ and $\psi \in C^{\infty}\left(\pi_{0}\right)$, (resp. $\left.\psi \in C^{\omega}\left(\pi_{0}\right)\right)$ the map $g \longmapsto d \pi_{0}\left(Z_{1}\right) \ldots d \pi_{0}\left(Z_{r}\right) \pi_{0}(g) \psi$ is $C^{\infty}$ (resp. analytic).

Proof. We prove only the second statement. That $i d \pi_{0}(Z)$ is essentially self adjoint on $C^{\infty}\left(\pi_{0}\right)$ and on $C^{\omega}\left(\pi_{0}\right)$ is immediate from Lemma 1. Using the 
adjoint representation we have, for any $Z \in \mathfrak{g}_{0}, g Z=\sum_{i} c_{i}(g) W_{i}$ for $g \in G_{0}$ where the $c_{i}$ are analytic functions on $G_{0}$ and $W_{i} \in \mathfrak{g}_{0}$. Hence, as

$$
d \pi_{0}(Z) \pi_{0}(g)=\pi_{0}(g) d \pi_{0}\left(g^{-1} Z\right)
$$

we can write $d \pi_{0}\left(Z_{1}\right) \ldots d \pi_{0}\left(Z_{r}\right) \pi_{0}(g) \psi$ as a linear combination with analytic coefficients of $\pi_{0}(g) d \pi_{0}\left(R_{1}\right) \ldots d \pi_{0}\left(R_{r}\right) \psi$ for suitable $R_{j} \in \mathfrak{g}_{0}$. The result is then immediate.

Definition $2 A$ unitary representation (UR) of a $S L G\left(G_{0}, \mathfrak{g}\right)$ is a triple $\left(\pi_{0}, \rho, \mathcal{H}\right), \mathcal{H}$ a SHS, with the following properties.

(a) $\pi_{0}$ is an even $U R$ of $G_{0}$ in $\mathcal{H}$;

(b) $\rho(X \longmapsto \rho(X))$ is a linear map of $\mathfrak{g}_{1}$ into $\operatorname{End}\left(C^{\infty}\left(\pi_{0}\right)\right)_{1}$ such that

(i) $\rho\left(g_{0} X\right)=\pi_{0}\left(g_{0}\right) \rho(X) \pi_{0}\left(g_{0}\right)^{-1}\left(X \in \mathfrak{g}_{1}, g_{0} \in G_{0}\right)$,

(ii) $\rho(X)$ with domain $C^{\infty}\left(\pi_{0}\right)$ is symmetric for all $X \in \mathfrak{g}_{1}$,

$$
\text { (iii) }-i d \pi_{0}([X, Y])=\rho(X) \rho(Y)+\rho(Y) \rho(X)\left(X, Y \in \mathfrak{g}_{1}\right) \text { on } C^{\infty}\left(\pi_{0}\right) \text {. }
$$

Proposition 1 If $\left(\pi_{0}, \rho, \mathcal{H}\right)$ is a UR of the SLG $\left(G_{0}, \mathfrak{g}\right)$, then $\rho(X)$ with domain $C^{\infty}\left(\pi_{0}\right)$ is essentially self adjoint for all $X \in \mathfrak{g}_{1}$. Moreover

$$
\pi: X_{0}+X_{1} \longmapsto d \pi_{0}\left(X_{0}\right)+\zeta^{-1} \rho\left(X_{1}\right) \quad\left(X_{i} \in \mathfrak{g}_{i}\right)
$$

is a representation of $\mathfrak{g}$ in $C^{\infty}\left(\pi_{0}\right)$.

Proof. Let $Z=(1 / 2)[X, X]$. We apply Lemma 1 with $U(t)=\pi_{0}(\exp t Z)=$ $e^{i t H}, \mathcal{B}=C^{\infty}\left(\pi_{0}\right)$. Then $H=-i d \pi_{0}(Z)=\rho(X)^{2}$ on $C^{\infty}\left(\pi_{0}\right)$. We conclude that $H$ and $\rho(X)$ are essentially self adjoint on $C^{\infty}\left(\pi_{0}\right)$ and that $H=\overline{\rho(X)}^{2}$; in particular, $H \geq 0$. For the second assertion the only non obvious statement is that for $Z \in \mathfrak{g}_{0}, X \in \mathfrak{g}_{1}, \psi \in C^{\infty}\left(\pi_{0}\right)$,

$$
\rho([Z, X]) \psi=d \pi_{0}(Z) \rho(X) \psi-\rho(X) d \pi_{0}(Z) \psi .
$$

Let $g_{t}=\exp (t Z)$ and let $\left(X_{k}\right)$ be a basis for $\mathfrak{g}_{1}$. Then $g X=\sum_{k} c_{k}(g) X_{k}$ where the $c_{k}$ are smooth functions on $G_{0}$. So

$$
\pi_{0}\left(g_{t}\right) \rho(X) \psi=\rho\left(g_{t} X\right) \pi_{0}\left(g_{t}\right) \psi=\sum_{k} c_{k}\left(g_{t}\right) \rho\left(X_{k}\right) \pi_{0}\left(g_{t}\right) \psi
$$

Now $g \longmapsto \pi_{0}(g) \psi$ is a smooth map into $C^{\infty}\left(\pi_{0}\right)$. On the other hand, if $H_{k}=-(1 / 2)\left[X_{k}, X_{k}\right]$, we have $i d \pi_{0}\left(H_{k}\right)=\rho\left(X_{k}\right)^{2}$ on $C^{\infty}\left(\pi_{0}\right)$, so $\pi_{0}(g) \psi \in$ 
$D\left(\rho\left(X_{k}\right)^{2}\right)$, and by Lemma \& $\rho\left(X_{k}\right)^{2} \pi_{0}(g) \psi=i d \pi_{0}\left(H_{k}\right) \pi_{0}(g) \psi$ is smooth in $g$. Lemma 2 now applies and shows that $\rho\left(X_{k}\right) \pi_{0}\left(g_{t}\right) \psi$ is smooth in $t$ and

$$
\left(\frac{d}{d t}\right)_{t=0} \rho\left(X_{k}\right) \pi_{0}\left(g_{t}\right) \psi=\rho\left(X_{k}\right) d \pi_{0}(Z) \psi .
$$

Hence

$$
d \pi_{0}(Z) \rho(X) \psi=\sum_{k} c_{k}(1) \rho\left(X_{k}\right) d \pi_{0}(Z) \psi+\sum_{k}\left(Z c_{k}\right)(1) \rho\left(X_{k}\right) \psi
$$

Since

$$
[Z, X]=\sum_{k}\left(Z c_{k}\right)(1) X_{k}, \quad X=\sum_{k} c_{k}(1) X_{k}
$$

the right side is equal to

$$
\rho(X) d \pi_{0}(Z) \psi+\rho([Z, X]) \psi
$$

Remark 1 For $Z$ such that $\exp t Z$ represents time translation, $H$ is the energy operator, and so is positive in the supersymmetric model.

We shall now show that one may replace $C^{\infty}\left(\pi_{0}\right)$ by a more or less arbitrary domain without changing the content of the definition. This shows that the concept of a UR of a SLG is a viable one even in the infinite dimensional context.

Let us consider a system $\left(\pi_{0}, \rho, \mathcal{B}, \mathcal{H}\right)$ with the following properties.

(a) $\mathcal{B}$ is a dense super linear subspace of $\mathcal{H}$ invariant under $\pi_{0}$ and $\mathcal{B} \subset$ $D\left(d \pi_{0}(Z)\right)$ for all $Z \in\left[\mathfrak{g}_{1}, \mathfrak{g}_{1}\right]$;

(b) $(\rho(X))_{X \in \mathfrak{g}_{1}}$ is a set of linear operators such that:

(i) $\rho(X)$ is symmetric for all $X \in \mathfrak{g}_{1}$,

(ii) $\mathcal{B} \subset D(\rho(X))$ for all $X \in \mathfrak{g}_{1}$,

(iii) $\rho(X) \mathcal{B}_{i} \subset \mathcal{H}_{i+1(\bmod 2)}$ for all $X \in \mathfrak{g}_{1}$,

(iv) $\rho(a X+b Y)=a \rho(X)+b \rho(Y)$ on $\mathcal{B}$ for $X, Y \in \mathfrak{g}_{1}$ and $a, b$ scalars,

(v) $\pi_{0}(g) \rho(X) \pi_{0}(g)^{-1}=\rho(g X)$ on $\mathcal{B}$ for all $g \in G_{0}, X \in \mathfrak{g}_{1}$,

(vi) $\rho(X) \mathcal{B} \subset D(\rho(Y))$ for all $X, Y \in \mathfrak{g}_{1}$, and $-i d \pi_{0}([X, Y])=\rho(X) \rho(Y)+$ $\rho(Y) \rho(X)$ on $\mathcal{B}$. 
Proposition 2 Let $\left(\pi_{0}, \rho, \mathcal{B}, \mathcal{H}\right)$ be as above. We then have the following.

(a) For any $X \in \mathfrak{g}_{1}, \rho(X)$ is essentially self adjoint and $C^{\infty}\left(\pi_{0}\right) \subset D(\overline{\rho(X)})$.

(b) Let $\bar{\rho}(X)=\left.\overline{\rho(X)}\right|_{C^{\infty}\left(\pi_{0}\right)}$ for $X \in \mathfrak{g}_{1}$. Then $\left(\pi_{0}, \bar{\rho}, \mathcal{H}\right)$ is a UR of the SLG $\left(G_{0}, \mathfrak{g}\right)$.

If $\left(\pi_{0}, \rho^{\prime}, \mathcal{H}\right)$ is a UR of the SLG $\left(G_{0}, \mathfrak{g}\right)$, such that $\mathcal{B} \subset D\left(\overline{\rho^{\prime}(X)}\right)$ and $\overline{\rho^{\prime}(X)}$ restricts to $\rho(X)$ on $\mathcal{B}$ for all $X \in \mathfrak{g}_{1}$, then $\rho^{\prime}=\bar{\rho}$.

Proof. Let $X \in \mathfrak{g}_{1}$. By assumption $\mathcal{B}$ is invariant under the one parameter unitary group generated by $H=-(1 / 2) i d \pi_{0}([X, X])$ while $H=\rho(X)^{2}$ on $\mathcal{B}$. So, by Lemma $\rho(X)$ is essentially self adjoint, $\overline{\rho(X)}=\overline{\left.\rho(X)\right|_{\mathcal{B}}}$, $H=(\overline{\rho(X)})^{2}$, and $D(H) \subset D(\overline{\rho(X)})$. Since $C^{\infty}\left(\pi_{0}\right) \subset D(H)$, we have proved (a).

Let us now prove (b). If $a$ is scalar and $X \in \mathfrak{g}, \bar{\rho}(a X)=a \bar{\rho}(X)$ follows from item (iv) and the fact that $\overline{\rho(X)}=\overline{\left.\rho(X)\right|_{\mathcal{B}}}$. For the additivity of $\bar{\rho}$, let $X, Y \in \mathfrak{g}_{1}$. Then $\rho(X+Y)$ is essentially self adjoint and its closure restricts to $\bar{\rho}(X+Y)$ on $C^{\infty}\left(\pi_{0}\right)$. Then, viewing $\overline{\rho(X)}+\overline{\rho(Y)}$ as a symmetric operator defined on the intersection of the domains of the two operators (which includes $C^{\infty}\left(\pi_{0}\right)$ ), we see that $\overline{\rho(X)}+\overline{\rho(Y)}$ is a symmetric extension of $\left.\rho(X)\right|_{\mathcal{B}}+\left.\rho(Y)\right|_{\mathcal{B}}=\left.\rho(X+Y)\right|_{\mathcal{B}}$. But as $\left.\rho(X+Y)\right|_{\mathcal{B}}$ is essentially self adjoint, we have, by the remark made earlier,

$$
\overline{\rho(X)}+\overline{\rho(Y)} \prec \overline{\left.\rho(X+Y)\right|_{\mathcal{B}}}=\overline{\rho(X+Y)} .
$$

Restricting both of these operators to $C^{\infty}\left(\pi_{0}\right)$ we find that $\bar{\rho}(X+Y)=$ $\bar{\rho}(X)+\bar{\rho}(Y)$. From the relation $\pi_{0}(g) \rho(X) \pi_{0}(g)^{-1}=\rho(g X)$ on $\mathcal{B}$ follows $\pi_{0}(g) \bar{\rho}(X) \pi_{0}(g)^{-1}=\bar{\rho}(g X)$.

The key step is now to prove that for any $X \in \mathfrak{g}_{1}, \overline{\rho(X)}$ maps $C^{\infty}\left(\pi_{0}\right)$ into itself. Fix $X \in \mathfrak{g}_{1}, \psi \in C^{\infty}\left(\pi_{0}\right)$. Now

$$
\pi_{0}(g) \overline{\rho(X)} \psi=\overline{\rho(g X)} \pi_{0}(g) \psi .
$$

So, writing $g X=\sum_{k} c_{k}(g) X_{k}$ where the $c_{k}$ are smooth functions on $G_{0}$ and the $\left(X_{k}\right)$ a basis for $\mathfrak{g}_{1}$, we have, remembering the linearity of $\bar{\rho}$ on $C^{\infty}\left(\pi_{0}\right)$,

$$
\pi_{0}(g) \overline{\rho(X)} \psi=\sum_{k} c_{k}(g) \overline{\rho\left(X_{k}\right)} \pi_{0}(g) \psi .
$$

It is thus enough to show that $g \longmapsto \overline{\rho\left(X_{k}\right)} \pi_{0}(g) \psi$ is smooth. If $H_{k}=$

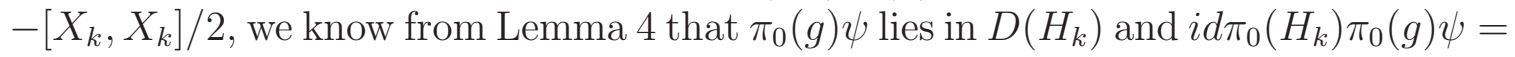


${\overline{\rho\left(X_{k}\right)}}^{2} \pi_{0}(g) \psi$ is smooth in $g$. Lemma 2 now shows that $\overline{\rho\left(X_{k}\right)} \pi_{0}(g) \psi$ is smooth in $g$.

It remains only to show that for $X, Y \in \mathfrak{g}_{1}$ we have

$$
-i d \pi_{0}([X, Y])=\bar{\rho}(X) \bar{\rho}(Y)+\bar{\rho}(Y) \bar{\rho}(X)
$$

on $C^{\infty}\left(\pi_{0}\right)$. But, the right side is $\bar{\rho}(X+Y)^{2}-\bar{\rho}(X)^{2}-\bar{\rho}(Y)^{2}$ while the left side is the restriction of $(-i / 2) d \pi_{0}([X+Y, X+Y])+(i / 2) d \pi_{0}([X, X])+$ $(i / 2) d \pi_{0}([Y, Y])$ to $C^{\infty}\left(\pi_{0}\right)$, and so we are done.

We must show the uniqueness of $\bar{\rho}$. Let $\rho^{\prime}$ have the required properties also. Then $\rho^{\prime}(X)$ is essentially self adjoint on $C^{\infty}\left(\pi_{0}\right)$ and $\mathcal{B}$ is a core for its closure, by Lemma 1. Hence $\rho^{\prime}(X)=\bar{\rho}(X)$. The proof is complete.

We shall now prove a variant of the above result involving analytic vectors.

Proposition 3 (i) If $\left(\pi_{0}, \rho, \mathcal{H}\right)$ is a UR of the $\operatorname{SLG}\left(G_{0}, \mathfrak{g}\right)$, then $\rho(X)$ maps $C^{\omega}\left(\pi_{0}\right)$ into itself for all $X \in \mathfrak{g}_{1}$, so that $\pi$, as in Proposition (1), is a representation of $\mathfrak{g}$ in $C^{\infty}\left(\pi_{0}\right)$. (ii) Let $G_{0}$ be connected. Let $\pi_{0}$ be an even unitary representation of $G_{0}$ and $\mathcal{B} \subset C^{\omega}\left(\pi_{0}\right)$ a dense linear super subspace. Let $\pi$ be a representation of $\mathfrak{g}$ in $\mathcal{B}$ such that $\pi(Z) \prec d \pi_{0}(Z)$ for $Z \in \mathfrak{g}_{0}$ and $\rho(X)=\zeta \pi(X)$ is symmetric for $X \in \mathfrak{g}_{1}$. Then, for each $X \in \mathfrak{g}_{1}$, $\rho(X)$ is essentially self adjoint on $\mathcal{B}$ and $C^{\infty}\left(\pi_{0}\right) \subset D(\overline{\rho(X)})$. If $\bar{\rho}(X)$ is the restriction of $\overline{\rho(X)}$ to $C^{\infty}\left(\pi_{0}\right)$, then $\left(\pi_{0}, \bar{\rho}, \mathcal{H}\right)$ is a UR of the $\operatorname{SLG}\left(G_{0}, \mathfrak{g}\right)$ and is the unique one in the following sense: if $\left(\pi_{0}, \rho^{\prime}, \mathcal{H}\right)$ is a UR with $\mathcal{B} \subset D\left(\overline{\rho^{\prime}(X)}\right)$ and $\left.\rho^{\prime}(X)\right|_{\mathcal{B}}=\rho(X)$ for all $X \in \mathfrak{g}_{1}$, then $\rho^{\prime}=\bar{\rho}$.

Proof. (i) This is proved as its $C^{\infty}$ analogue in the proof of Proposition 2, using the analytic parts of Lemmas 2 and 4

(ii) The proof that $\rho(X)$ for $X \in \mathfrak{g}_{1}$ is essentially self adjoint with $D(\overline{\rho(X)}) \supset C^{\infty}\left(\pi_{0}\right)$ follows as before from (the analytic part of) Lemma 11. The same goes for the linearity of $\bar{\rho}$.

We shall now show that for $X \in \mathfrak{g}_{1}, g \in G_{0}$,

$$
\pi_{0}\left(g^{-1}\right) \overline{\rho(X)} \pi_{0}(g)=\overline{\rho\left(g^{-1} X\right)} .
$$

Write $g^{-1} X=\sum_{k} c_{k}(g) X_{k}$ where $\left(X_{k}\right)$ is a basis for $\mathfrak{g}_{1}$ and the $c_{k}$ are analytic functions on $G_{0}$. We begin by showing that for all $\psi \in \mathcal{B}$

$$
\overline{\rho(X)} \pi_{0}(g) \psi=\pi_{0}(g) \rho\left(g^{-1} X\right) \psi .
$$

Now

$$
\pi_{0}(g) \rho\left(g^{-1} X\right) \psi=\sum_{k} c_{k}(g) \pi_{0}(g) \rho\left(X_{k}\right) \psi
$$


We argue as in Proposition 2 to conclude, using Lemmas 2 and 4 that the function $\overline{\rho(X)} \pi_{0}(g) \psi$ is analytic in $g$ and its derivatives can be calculated explicitly. It is also clear that the right side is analytic in $g$ since $\rho\left(X_{k}\right) \psi \in \mathcal{B}$ for all $k$. So, as $G_{0}$ is connected, it is enough to prove that the two sides in (2) have all derivatives equal at $g=1$. This comes down to showing that for any integer $n \geq 0$ and any $Z \in \mathfrak{g}_{0}$,

$$
\rho(X) d \pi_{0}(Z)^{n} \psi=\sum_{k, r}\left(\begin{array}{l}
n \\
r
\end{array}\right)\left(Z^{r} c_{k}\right)(1) d \pi_{0}(Z)^{n-r} \rho\left(X_{k}\right) \psi
$$

Let $\lambda$ be the representation of $G_{0}$ on $\mathfrak{g}_{1}$ and write $\lambda$ again for $d \lambda$. Then, taking $g_{t}=\exp (t Z)$,

$$
\lambda\left(g_{t}^{-1}\right)(X)=\sum_{k} c_{k}\left(g_{t}\right) X_{k}
$$

from which we get, on differentiating $n$ times with respect to $t$ at $t=0$,

$$
\lambda(-Z)^{r}(X)=\sum_{k}\left(Z^{r} c_{k}\right)(1) X_{k}
$$

Hence the right side of (3) becomes

$$
\sum_{r}\left(\begin{array}{l}
n \\
r
\end{array}\right) d \pi_{0}(Z)^{n-r} \rho\left(\lambda(-Z)^{r}(X)\right) \psi .
$$

On the other hand, from the fact that $\pi$ is a representation of $\mathfrak{g}$ in $\mathcal{B}$ we get

$$
\rho(X) d \pi_{0}(Z)=d \pi_{0}(Z) \rho(X)+\rho(\lambda(-Z)(X))
$$

on $\mathcal{B}$. Iterating this we get, on $\mathcal{B}$,

$$
\rho(X) d \pi_{0}(Z)^{n}=\sum_{r}\left(\begin{array}{l}
n \\
r
\end{array}\right) d \pi_{0}(Z)^{n-r} \rho\left(\lambda(-Z)^{r}(X)\right)
$$

which gives (21). But then (11) follows from (2) by a simple closure argument.

Using (11), the proof that $\bar{\rho}(X)$ maps $C^{\infty}\left(\pi_{0}\right)$ into itself is the same of Proposition [2. The proof of the relation $-i d \pi_{0}([X, Y])=\bar{\rho}(X) \bar{\rho}(Y)+$ $\bar{\rho}(Y) \bar{\rho}(X)$ for $X, Y \in \mathfrak{g}_{1}$ is also the same. The proof is complete. 


\subsection{The category of unitary representations of a super Lie group}

If $\Pi=\left(\pi_{0}, \rho, \mathcal{H}\right)$ and $\Pi^{\prime}=\left(\pi_{0}^{\prime}, \rho^{\prime}, \mathcal{H}^{\prime}\right)$ are two UR's of a SLG $\left(G_{0}, \mathfrak{g}\right)$, a morphism $A: \Pi \longrightarrow \Pi^{\prime}$ is an even bounded linear operator from $\mathcal{H}$ to $\mathcal{H}^{\prime}$ such that $A$ intertwines $\pi_{0}, \rho$ and $\pi_{0}^{\prime}, \rho^{\prime}$; notice that as soon as $A$ intertwines $\pi_{0}$ and $\pi_{0}^{\prime}$, it maps $C^{\infty}\left(\pi_{0}\right)$ into $C^{\infty}\left(\pi_{0}^{\prime}\right)$, and so the requirement that it intertwine $\rho$ and $\rho^{\prime}$ makes sense. An isomorphism is then a morphism $A$ such that $A^{-1}$ is a bounded operator; in this case $A$ is a linear isomorphism of $C^{\infty}\left(\pi_{0}\right)$ with $C^{\infty}\left(\pi_{0}^{\prime}\right)$ intertwining $\rho$ and $\rho^{\prime}$. If $A$ is unitary we then speak of unitary equivalence of $\Pi$ and $\Pi^{\prime}$. It is easily checked that equivalence implies unitary equivalence, just as in the classical case. $\Pi^{\prime}$ is a subrepresentation of $\Pi$ if $\mathcal{H}^{\prime}$ is a closed graded subspace of $\mathcal{H}$ invariant under $\pi_{0}$ and $\rho$, and $\pi_{0}^{\prime}$ (resp. $\left.\rho^{\prime}\right)$ is the restriction of $\pi_{0}$ (resp. $\rho$ ) to $\mathcal{H}^{\prime}\left(\right.$ resp. $\left.C^{\infty}\left(\pi_{0}\right) \cap \mathcal{H}^{\prime}\right)$. The UR $\Pi$ is said to be irreducible if there is no proper nonzero closed graded subspace $\mathcal{H}^{\prime}$ that defines a subrepresentation. If $\Pi^{\prime}$ is a nonzero proper subrepresentation of $\Pi$, and $\mathcal{H}^{\prime \prime}$ is $\mathcal{H}^{\perp}$, it follows from the self adjointness of $\rho(X)$ for $X \in \mathfrak{g}_{1}$ that $\mathcal{H}^{\prime \prime} \cap C^{\infty}\left(\pi_{0}\right)$ is invariant under all $\rho(X)\left(X \in \mathfrak{g}_{1}\right)$; then the restrictions of $\pi_{0}, \rho$ to $\mathcal{H}^{\prime \prime}$ define a subrepresentation $\Pi^{\prime \prime}$ such that $\Pi=\Pi^{\prime} \oplus \Pi^{\prime \prime}$ in an obvious manner.

Lemma $5 \Pi$ is irreducible if and only if $\operatorname{Hom}(\Pi, \Pi)=\mathbb{C}$.

Proof. If $\Pi$ splits as above, then the orthogonal projection $\mathcal{H} \longrightarrow \mathcal{H}^{\prime}$ is a nonscalar element of $\operatorname{Hom}(\Pi, \Pi)$. Conversely, suppose that $\Pi$ is irreducible and $A \in \operatorname{Hom}(\Pi, \Pi)$. Then $A^{*} \in \operatorname{Hom}(\Pi, \Pi)$ also and so, to prove that $A$ is a scalar we may suppose that $A$ is self adjoint. Let $P$ be the spectral measure of $A$. Clearly all the $P(E)$ are even. Then $P$ commutes with $\pi_{0}$ and so $P(E)$ leaves $C^{\infty}\left(\pi_{0}\right)$ invariant for all Borel sets $E$. Moreover, by Lemma 3, the relation $A \rho(X)=\rho(X) A$ on $C^{\infty}\left(\pi_{0}\right)$ implies that $P(E) \leftrightarrow \overline{\rho(X)}$ for all $E$ and $x \in \mathfrak{g}_{1}$, and hence that $P(E) \rho(X)=\rho(X) P(E)$ on $C^{\infty}\left(\pi_{0}\right)$ for all $E, X$. The range of $P(E)$ thus defines a subrepresentation and so $P(E)=0$ or $I$. Since this is true for all $E, A$ must be a scalar.

Lemma 6 Let $\left(R_{0}, \mathfrak{r}\right)$ be a SLG and $\left(\theta, \rho^{\theta}\right)$ a UR of it, in a Hilbert space $\mathcal{L}$. Let $P^{X}$ be the spectral measure of $\overline{\rho^{\theta}(X)}, X \in \mathfrak{r}_{1}$. Then the following properties of a closed linear subspace $\mathcal{M}$ of $\mathcal{L}$ are equivalent: $(i) \mathcal{M}$ is stable under $\theta$ and $\mathcal{M}^{\infty}=C^{\infty}(\theta) \cap \mathcal{M}$ is stable under all $\rho^{\theta}(X),\left(X \in \mathfrak{r}_{1}\right)$ (ii) $\mathcal{M}$ is stable under $\theta$ and all the spectral projections $P_{F}^{X}$ (Borel $F \subset \mathbb{R}$ ). In particular, $\left(\theta, \rho^{\theta}\right)$ is irreducible if and only if $\mathcal{L}$ is irreducible under $\theta$ and all $P_{F}^{X}$. 
Proof. Follows from Lemma 3 applied to the orthogonal projection $L$ : $\mathcal{L} \longrightarrow \mathcal{M}$. Indeed, suppose that $\mathcal{M}$ is a closed linear subspace of $\mathcal{L}$ stable under $\theta$. Then $L$ maps $\mathcal{L}^{\infty}$ onto $\mathcal{M}^{\infty}$. By Lemma $3 L$ commutes with $\rho^{\theta}(X)$ on $\mathcal{L}^{\infty}$ if and only if $L \leftrightarrow \overline{\rho^{\theta}(X)}$; this is the same as saying that $P^{X}$ stabilizes $\mathcal{M}$

\section{Induced representations of super Lie groups, super systems of imprimitivity, and the su- per imprimitivity theorem}

\subsection{Smooth structure of the classical induced repre- sentation and its system of imprimitivity}

Let $G_{0}$ be a unimodular Lie group and $H_{0}$ a closed Lie subgroup. We write $\Omega=G_{0} / H_{0}$ and assume that $\Omega$ has a $G_{0}$-invariant measure; one can easily modify the treatment below to avoid these assumptions. We write $x \mapsto \bar{x}$ for the natural map from $G_{0}$ to $\Omega$ and $d \bar{x}$ for a choice of the invariant measure on $\Omega$. For any UR $\sigma$ of $H_{0}$ in a Hilbert space $\mathcal{K}$ one has the representation $\pi$ of $G_{0}$ induced by $\sigma$. One may take $\pi$ as acting in the Hilbert space $\mathcal{H}$ of (equivalence classes) of Borel functions $f$ from $G_{0}$ to $\mathcal{K}$ such that (i) $f(x \xi)=\sigma(\xi)^{-1} f(x)$ for all $x \in G_{0}, \xi \in H_{0}$, and (ii) $\|f\|_{\mathcal{H}}^{2}:=\int_{\Omega}|f(x)|_{\mathcal{K}}^{2} d \bar{x}<\infty$. Here $|f(x)|_{\mathcal{K}}$ is the norm of $f(x)$ in $\mathcal{K}$, and the function $x \mapsto|f(x)|_{\mathcal{K}}^{2}$ is defined on $\Omega$ so that it makes sense to integrate it on $\Omega$. Let $P$ be the natural projection valued measure on $\mathcal{H}$ defined as follows: for any Borel set $E \subset \Omega$ the projection $P(E)$ is the operator $f \mapsto \chi_{E} f$ where $\chi_{E}$ is the characteristic function of $E$. Then $(\pi, \mathcal{H}, P)$ is the classical system of imprimitivity (SI) associated to the UR $\sigma$ of $H_{0}$. In our case $G_{0}$ is a Lie group and it is better to work with a smooth version of $\pi$; its structure is determined by a well known theorem of Dixmier-Malliavin in a manner that will be explained below.

We begin with a standard but technical lemma that says that certain integrals containing a parameter are smooth.

Lemma 7 Let $M, N$ be smooth manifolds, $d n$ a smooth measure on $N$, and $B$ a separable Banach space with norm $|\cdot|$. Let $F: M \times N \longrightarrow B$ be a map with the following properties: (i) For each $n \in N, m \mapsto F(m, n)$ is smooth (ii) If $A \subset M$ is an open set with compact closure, and $G$ is any derivative of $F$ with respect to $m$, there is a $g_{A} \in L^{1}(N, d n)$ such that $|G(m, n)| \leq g_{A}(n)$ 
for all $m \in A, n \in N$. Then

$$
f(m)=\int_{N} F(m, n) d n
$$

exists for all $m$ and $f$ is a smooth map of $M$ into $B$.

Proof. It is a question of proving that the integrals

$$
\int_{N}|G(m, n)| d n
$$

converge uniformly when $m$ varies in an open subset $A$ of $M$ with compact closure. But the integrand is majorized by $g_{A}$ which is integrable on $N$ and so the uniform convergence is clear.

We also observe that any $f \in \mathcal{H}$ lies in $L^{p \text { loc }}\left(G_{0}\right)$ for $p=1,2$, i.e., $\theta(x)|f(x)|_{\mathcal{K}}^{2}$ is integrable on $G_{0}$ for any continuous compactly supported scalar function $\theta \geq 0$. In fact

$$
\int_{G_{0}} \theta(x)|f(x)|_{\mathcal{K}}^{2} d x=\int_{\Omega}\left(\int_{H_{0}} \theta(x \xi)|f(x \xi)|_{\mathcal{K}}^{2} d \xi\right) d \bar{x}=\int_{\Omega} \bar{\theta}(x)|f(x)|_{\mathcal{K}}^{2} d \bar{x}<\infty
$$

where $\bar{\theta}(x)=\int_{G_{0}} \theta(x \xi) d \xi$.

In $\mathcal{H}$ we have the space $C^{\infty}(\pi)$ of smooth vectors for $\pi$. We also have its Garding subspace, the subspace spanned by all vectors $\pi(\alpha) h$ where $\alpha \in$ $C_{c}^{\infty}\left(G_{0}\right)$ and $h \in \mathcal{H}$. We have

$$
(\pi(\alpha) h)(z)=\int_{G_{0}} \alpha(x) h\left(x^{-1} z\right) d x=\int_{G_{0}} \alpha\left(z t^{-1}\right) h(t) d t \quad\left(z \in G_{0}\right) .
$$

The integrals exist because $h$ is locally $L^{2}$ on $G_{0}$ as mentioned above. Since $h \in L^{1, \operatorname{loc}}\left(G_{0}\right), \alpha \in C_{c}^{\infty}\left(G_{0}\right)$, the conditions of Lemma 7 are met and so $\pi(\alpha) h$ is smooth. Thus all elements of the Garding space are smooth functions. But the Dixmier-Malliavin theorem asserts that the Garding space is exactly the same as $C^{\infty}(\pi)$ DM78. Thus all elements of $C^{\infty}(\pi)$ are smooth functions from $G_{0}$ to $\mathcal{K}$. This is the key point that leads to the smooth versions of the induced representation and the SI at the classical level.

Let us define $\mathcal{B}$ as the space of all functions $f$ from $G_{0}$ to $\mathcal{K}$ such that (i) $f$ is smooth and $f(x \xi)=\sigma(\xi)^{-1} f(x)$ for all $x \in G_{0}, \xi \in H_{0}$ (ii) $f$ has compact support $\bmod H_{0}$. Let $C_{c}^{\infty}(\pi)$ be the subspace of all elements of $C^{\infty}(\pi)$ with compact support $\bmod H_{0}$.

Proposition $4 \mathcal{B}$ has the following properties: (i) $\mathcal{B}=C_{c}^{\infty}(\pi)$ (ii) $\mathcal{B}$ is dense in $\mathcal{H}($ iii $) f(x) \in C^{\infty}(\sigma)$ for all $x \in G_{0}($ iv $) \mathcal{B}$ is stable under $d \pi$. 
Proof. (i) Let $f \in \mathcal{B}$. To show that $f \in C^{\infty}(\pi)$ it is enough to show that for any $u \in \mathcal{H}$ the map $x \mapsto\left(\pi\left(x^{-1}\right) f, u\right)_{\mathcal{H}}$ is smooth in $x$. Now

$$
\left(\pi\left(x^{-1}\right) f, u\right)_{\mathcal{H}}=\int_{\Omega}(f(x y), u(y))_{\mathcal{K}} d \bar{y}
$$

Since $|u|_{\mathcal{K}}$ is locally $L^{1}$ on $X$ and $f$ is smooth, the conditions of Lemma 7 are met. We have $\mathcal{B} \subset C_{c}^{\infty}(\pi)$. The reverse inclusion is immediate from the Dixmier-Malliavin theorem, as remarked above.

(ii) It is enough to prove that any $h \in \mathcal{H}$ with compact support mod $H_{0}$ is in the closure of $\mathcal{B}$. We know that $\pi(\alpha) h \rightarrow h$ as $\alpha \in C_{c}^{\infty}\left(G_{0}\right)$ goes suitably to the delta function at the identity of $G_{0}$. But $\pi(\alpha) h$ is smooth and has compact support mod $H_{0}$ because $h$ has the same property, so that $\pi(\alpha) h \in \mathcal{B}$.

(iii) Fix $x \in G_{0}$. Since $\sigma(\xi) f(x)=f\left(x \xi^{-1}\right)$ for $\xi \in H_{0}$ it is clear that $f(x) \in C^{\infty}(\sigma)$.

(iv) Let $f \in \mathcal{B}, Z \in \mathfrak{g}_{0}$. Then

$$
(d \pi(Z) f)(x)=(d / d t)_{t=0} f(\exp (-t Z) x)
$$

is smooth and we are done.

We refer to $(\pi, \mathcal{B})$ as the smooth representation induced by $\sigma$. We shall also define the smooth version of the SI. For any $u \in C_{c}^{\infty}(\Omega)$ let $M(u)$ be the bounded operator on $\mathcal{H}$ which is multiplication by $u$. Then $M(u)$ leaves $\mathcal{B}$ invariant and $M: u \mapsto M(u)$ is a $*$-representation of the $*$-algebra $C_{c}^{\infty}(\Omega)$ in $\mathcal{H}$. It is natural to refer to $(\pi, \mathcal{B}, M)$ as the smooth system of imprimitivity associated to $\sigma$. Observe that $f \in C^{\infty}(\pi)$ has compact support $\bmod H_{0}$ if and only if there is some $u \in C_{c}^{\infty}(\Omega)$ such that $f=M(u) f$. Proposition 4 shows that $\mathcal{B}$ is thus determined intrinsically by the SI associated to $\sigma$. The passage from $(\pi, \mathcal{H}, P)$ to $(\pi, \mathcal{B}, M)$ is thus functorial and is a categorical equivalence. Thus we are justified in working just with smooth SI's.

It is easy to see that the assignment that takes $\sigma$ to the associated smooth $\mathrm{SI}$ is functorial. Indeed, let $R$ be a morphism from $\sigma$ to $\sigma^{\prime}$, i.e., $R$ is a bounded operator from $\mathcal{K}$ to $\mathcal{K}^{\prime}$ intertwining $\sigma$ and $\sigma^{\prime}$. We then define $T_{R}=$ $T\left(\mathcal{H} \longrightarrow \mathcal{H}^{\prime}\right)$ by $\left(T_{R} f\right)(x)=R f(x)\left(x \in G_{0}\right)$. It is then immediate that $T_{R}$ is a morphism from the (smooth) SI associated to $\sigma$ to the (smooth) SI associated to $\sigma^{\prime}$. This functor is an equivalence of categories. To verify this one must show that every morphism between the two SI's is of this form. This is of course classical but we sketch the argument depending on the following lemma which will be essentially used in the super context also. 
Lemma 8 Suppose $f \in \mathcal{B}$ and $f(1)=0$. Then we can find $u_{i} \in C_{c}^{\infty}(\Omega), g_{i} \in$ $\mathcal{B}$ such that $(i) u_{i}(1)=0$ for all $i$ (ii) we have $f=\sum_{i} u_{i} g_{i}$.

Proof. If $f$ vanishes in a neighborhood of 1 , we can choose $u \in C_{c}^{\infty}(\Omega)$ such that $u=0$ in a neighborhood of 1 and $f=u f$. The result is thus true for $f$. Let $f \in \mathcal{B}$ be arbitrary but vanishing at 1 . Let $\mathfrak{z}$ be a linear subspace of $\mathfrak{g}_{0}=$ Lie $\left(G_{0}\right)$ complementary to $\mathfrak{h}_{0}=$ Lie $\left(H_{0}\right)$. Then there is a sufficiently small $r>0$ such that if $\mathfrak{z}_{r}=\{Z \in \mathfrak{z}|| Z \mid<r\},|\cdot|$ being a norm on $\mathfrak{z}$, the map

$$
\mathfrak{z}_{r} \times H_{0} \longrightarrow G_{0}, \quad(Z, \xi) \longmapsto \exp Z \cdot \xi
$$

is a diffeomorphism onto an open set $G_{1}=G_{1} H_{0}$ of $G_{0}$. We transfer $f$ from $G_{1}$ to a function, denoted by $\varphi$ on $\mathfrak{z}_{r} \times H_{0}$. We have $\varphi(0, \xi)=0$, and $\varphi\left(Z, \xi \xi^{\prime}\right)=\sigma\left(\xi^{\prime}\right)^{-1} \varphi(Z, \xi)$ for $\xi^{\prime} \in H_{0}$. If $t_{i}(1 \leq i \leq k)$ are the linear coordinates on $\mathfrak{z}$,

$$
\varphi(Z, \xi)=\sum_{i} t_{i}(Z) \int_{0}^{1}\left(\partial \varphi / \partial t_{i}\right)(s Z, \xi) d s .
$$

The functions $\psi_{i}(Z, \xi)=\int_{0}^{1}\left(\partial \varphi / \partial t_{i}\right)(s Z, \xi) d s$ are smooth by Lemma $\mathbf{Z}$ while $\psi_{i}\left(Z, \xi \xi^{\prime}\right)=\sigma\left(\xi^{\prime}\right)^{-1} \psi_{i}(Z, \xi)$ for $\xi^{\prime} \in H_{0}$. So, going back to $G_{1}$ we can write $f=\sum_{i} t_{i} h_{i}$ where $t_{i}$ are now in $C^{\infty}\left(G_{1}\right)$, right invariant under $H_{0}$ and vanishing at 1 , while the $h_{i}$ are smooth and satisfy $h_{i}(x \xi)=\sigma(\xi)^{-1} h_{i}(x)$ for $x \in G_{1}, \xi \in H_{0}$. If $u \in C_{c}^{\infty}(\Omega)$ is such that $u$ is 1 in a neighborhood of 1 and $\operatorname{supp}(u) \subset G_{1}$, then $u^{2} f=\sum_{i} u_{i} g_{i}$ where $u_{i}=u t_{i} \in C_{c}^{\infty}(\Omega), u_{i}(1)=0$, and $g_{i}=u h_{i} \in \mathcal{B}$. Since $f=u^{2} f+\left(1-u^{2}\right) f$ and $\left(1-u^{2}\right) f=0$ in a neighborhood of 1 , we are done.

We can now determine all the morphisms from $\mathcal{H}$ to $\mathcal{H}^{\prime}$. Let $T$ be a morphism $\mathcal{H} \longrightarrow \mathcal{H}^{\prime}$. Then, as $T$ commutes with multiplications by elements of $C_{c}^{\infty}(\Omega)$, it maps $\mathcal{B}$ to $\mathcal{B}^{\prime}$. Moreover, for the same reason, the above lemma shows that if $f \in \mathcal{B}$ and $f(1)=0$, then $(T f)(1)=0$. So the map

$$
R: f(1) \longmapsto(T f)(1) \quad(f \in \mathcal{B})
$$

is well defined. From the fact that $T$ intertwines $\pi$ and $\pi^{\prime}$ we obtain that $(T f)(x)=R f(x)$ for all $x \in G_{0}$. To complete the proof we must show two things: (1) $R$ is defined on all of $C^{\infty}(\sigma)$ and (2) $R$ is bounded. For (1), let $v \in C^{\infty}(\sigma)$. In the earlier notation, if $u \in C_{c}^{\infty}\left(G_{0}\right)$ is 1 in 1 and has support contained in $G_{1}$, then $h:(\exp Z, \xi) \mapsto u(\exp Z) \sigma(\xi)^{-1} v$ is in $\mathcal{B}$ and $h(1)=v$. For proving (2), let the constant $C>0$ be such that

$$
\|T g\|_{\mathcal{H}^{\prime}} \leq C\|g\|_{\mathcal{H}} \quad\left(g \in \mathcal{H}^{\prime}\right)
$$


Then, taking $g=u^{1 / 2} f$ for $f \in \mathcal{B}$ and $u \geq 0$ in $C_{c}^{\infty}(\Omega)$, we get

$$
\int_{\Omega} u(x)|R f(x)|_{\mathcal{K}}^{2} d \bar{x} \leq C \int_{\Omega} u(x)|f(x)|_{\mathcal{K}^{\prime}}^{2} d \bar{x}
$$

for all $f \in \mathcal{B}$ and $u \geq 0$ in $C_{c}^{\infty}(\Omega)$. So $|R f(x)|_{\mathcal{K}} \leq C|f(x)|_{\mathcal{K}}$ for almost all $x$. As $f$ and $R f=T f$ are continuous this inequality is valid for all $x$, in particular for $x=1$, proving that $R$ is bounded.

\subsection{Representations induced from a special sub super Lie group}

It is now our purpose to extend this smooth classical theory to the super context. A SLG $\left(H_{0}, \mathfrak{h}\right)$ is a sub super Lie group of the SLG $\left(G_{0}, \mathfrak{g}\right)$ if $H_{0} \subset$ $G_{0}, \mathfrak{h} \subset \mathfrak{g}$, and the action of $H_{0}$ on $\mathfrak{h}$ is the restriction of the action of $H_{0}$ (as a subgroup of $G_{0}$ ) on $\mathfrak{g}$. We shall always suppose that $H_{0}$ is closed in $G_{0}$. The sub SLG $\left(H_{0}, \mathfrak{h}\right)$ is called special if $\mathfrak{h}$ has the same odd part as $\mathfrak{g}$, i.e., $\mathfrak{h}_{1}=\mathfrak{g}_{1}$. In this case the super homogeneous space associated is purely even and coincides with $\Omega=G_{0} / H_{0}$. As in 3.1 we shall assume that $\Omega$ admits an invariant measure although it is not difficult to modify the treatment to avoid this assumption. Both conditions are satisfied in the case of the super Poincaré groups and their variants.

We start with a UR $\left(\sigma, \rho^{\sigma}, \mathcal{K}\right)$ of $\left(H_{0}, \mathfrak{h}\right)$ and associate to it the smooth induced representation $(\pi, \mathcal{B})$ of the classical group $G_{0}$. In our case $\mathcal{K}$ is a SHS and so $\mathcal{H}$ becomes a SHS in a natural manner, the parity subspaces being the subspaces where $f$ takes its values in the corresponding parity subspace of $\mathcal{K}$. $\pi$ is an even UR.

We shall now define the operators $\rho^{\pi}(X)$ for $X \in \mathfrak{g}_{1}$ as follows:

$$
\left(\rho^{\pi}(X) f\right)(x)=\rho^{\sigma}\left(x^{-1} X\right) f(x) \quad(f \in \mathcal{B}) .
$$

Since the values of $f$ are in $C^{\infty}(\sigma)$ the right side is well defined. In order to prove that the definition gives us an odd operator on $\mathcal{B}$ we need a lemma.

Lemma $9\left[\mathfrak{g}_{1}, \mathfrak{g}_{1}\right] \subset \mathfrak{h}_{0}$ and is stable under $G_{0}$. In particular it is an ideal in $\mathfrak{g}_{0}$.

Proof. For $g \in G_{0}, Y, Y^{\prime} \in \mathfrak{g}_{1}$, we have $g\left[Y, Y^{\prime}\right]=\left[g Y, g Y^{\prime}\right] \in\left[\mathfrak{g}_{1}, \mathfrak{g}_{1}\right]$. Since $\mathfrak{h}_{0} \oplus \mathfrak{g}_{1}$ is a super Lie algebra, $\left[\mathfrak{g}_{1}, \mathfrak{g}_{1}\right] \subset \mathfrak{h}_{0}$.

Proposition $5 \rho^{\pi}(X)$ is an odd linear map $\mathcal{B} \longrightarrow \mathcal{B}$ for all $X \in \mathfrak{g}_{1}$. Moreover $\rho^{\pi}(X)$ is local, i.e., $\operatorname{supp}\left(\rho^{\pi}(X) f\right) \subset \operatorname{supp}(f)$ for $f \in \mathcal{B}$. Finally, if $Z \in \mathfrak{h}_{0}$, we have $-d \sigma(Z) f(g)=(Z f)(g)$. 
Proof. The support relation is trivial. Further, for $x \in G_{0}, \xi \in H_{0}$,

$$
\begin{aligned}
\left(\rho^{\pi}(X) f\right)(x \xi) & =\rho^{\sigma}\left(\xi^{-1} x^{-1} X\right) f(x \xi) \\
& =\sigma(\xi)^{-1} \rho^{\sigma}\left(x^{-1} X\right) \sigma(\xi) f(x \xi) \\
& =\sigma(\xi)^{-1}\left(\rho^{\pi}(X) f\right)(x) .
\end{aligned}
$$

It is thus a question of proving that $g \mapsto \rho^{\sigma}\left(g^{-1} X\right) f(g)$ is smooth. If $\left(X_{k}\right)$ is a basis for $\mathfrak{g}_{1}, g^{-1} X=\sum_{k} c_{k}(g) X_{k}$ where the $c_{k}$ are smooth functions and so it is enough to prove that $g \mapsto \rho^{\sigma}(Y) f(g)$ is smooth for any $Y \in \mathfrak{g}, f \in \mathcal{B}$. We use Lemma 2 If $Z=(1 / 2)[Y, Y]$, we have $\rho^{\sigma}(Y)^{2} f(g)=-i d \sigma(Z) f(g)$, and we need only show that $-d \sigma(Z) f(g)$ is smooth in $g$. But $Z \in \mathfrak{h}_{0}$ and $f(g \exp t Z)=\sigma(\exp (-t Z)) f(g)$ so that $-d \sigma(Z) f(g)=(Z f)(g)$ is clearly smooth in $g$. Note that this argument applies to any $Z \in \mathfrak{h}_{0}$, giving the last assertion.

Proposition $6\left(\pi, \rho^{\sigma}, \mathcal{B}\right)$ is a UR of the SLG $\left(G_{0}, \mathfrak{g}\right)$.

Proof. The symmetry of $\rho^{\pi}(X)$ and the relations $\rho^{\pi}(y X)=\pi(y) \rho^{\pi}(X) \pi(y)^{-1}$ follow immediately from the corresponding relations for $\rho^{\sigma}$. Suppose now that $X, Y \in \mathfrak{g}_{1}$. Then $\left(\rho^{\pi}(X) \rho^{\pi}(Y) f\right)(x)=\rho^{\sigma}\left(x^{-1} X\right) \rho^{\sigma}\left(x^{-1} Y\right) f(x)$. Hence

$$
\begin{aligned}
& \left(\left(\rho^{\pi}(X) \rho^{\pi}(Y)+\rho^{\pi}(Y) \rho^{\pi}(X)\right) f\right)(x)=-i d \sigma\left(x^{-1}[X, Y]\right) f(x) \\
& =i\left(x^{-1}[X, Y] f\right)(x)(\text { Proposition } 15) \\
& =i(d / d t)_{t=0} f\left(x\left(x^{-1} \exp t[X, Y] x\right)\right) \\
& =i(d / d t)_{t=0} f(\exp t[X, Y] x) \\
& =i(d / d t)_{t=0}(\pi(\exp (-t[X, Y]) f)(x) \\
& =-i(d \pi([X, Y]) f)(x) \text {. }
\end{aligned}
$$

This proves the proposition. We refer to $\left(\pi, \rho^{\pi}, \mathcal{H}\right)$ as the UR of the SLG $\left(G_{0}, \mathfrak{g}\right)$ induced by the UR $\left(\sigma, \rho^{\sigma}, \mathcal{K}\right)$ of $\left(H_{0}, \mathfrak{h}\right)$, and to $\left(\pi, \rho^{\pi}, \mathcal{B}\right)$ as the corresponding smooth induced UR.

Write $P$ for the natural projection valued measure in $\mathcal{H}$ based on $\Omega$ : for any Borel $E \subset \Omega, P(E)$ is the operator in $\mathcal{H}$ of multiplication by $\chi_{E}$, the characteristic function of $E$.

Recall the definition of $\leftrightarrow$ before Lemma 3

Proposition 7 For $X \in \mathfrak{g}_{1}$, and $u \in C_{c}^{\infty}(\Omega)$, we have $M(u) \leftrightarrow \overline{\rho^{\pi}(X)}$. Furthermore $P(E) \leftrightarrow \overline{\rho^{\pi}(X)}$ for Borel $E \subset \Omega$. 
Proof. It is standard that a bounded operator commutes with all $P(E)$ if and only if it commutes with all $M(u)$ for $u \in C_{c}^{\infty}(\Omega)$. It is thus enough to prove that $M(u) \leftrightarrow \overline{\rho^{\pi}(X)}$ for all $u, X$. On $\mathcal{B}$ we have $M(u) \rho^{\pi}(X)=\rho^{\pi}(X) M(u)$ trivially from the definitions, and so we are done in view of Lemma 3 .

Theorem 1 The assignment that takes $\left(\sigma, \rho^{\sigma}\right)$ to $\left(\pi, \rho^{\pi}, \mathcal{B}, M\right)$ is a fully faithful functor.

Proof. Let $R$ be a morphism intertwining $\left(\sigma, \rho^{\sigma}\right)$ and $\left(\sigma^{\prime}, \rho^{\sigma^{\prime}}\right)$, and let $T$ : $\mathcal{B} \longrightarrow \mathcal{B}^{\prime}$ be associated to $R$ such that $(T f)(x)=R f(x)$. It is then immediate that $T$ intertwines $\rho^{\pi}$ and $\rho^{\pi^{\prime}}$. Conversely, if $T$ is a morphism between the induced systems, from the classical discussion following Lemma 8 we know that $(T f)(x)=R f(x)$ for a bounded even operator $R$ intertwining $\sigma$ and $\sigma^{\prime}$. Since $T$ intertwines $\rho^{\pi}$ and $\rho^{\pi^{\prime}}$ we conclude that $R$ must intertwine $\rho^{\sigma}$ and $\rho^{\sigma^{\prime}}$.

\subsection{Super systems of imprimitivity and the super im- primitivity theorem}

A super system of imprimitivity (SSI) based on $\Omega$ is a collection $\left(\pi, \rho^{\pi}, \mathcal{H}, P\right)$ where $\left(\pi, \rho^{\pi}, \mathcal{H}\right)$ is a UR of the $\operatorname{SLG}\left(G_{0}, \mathfrak{g}\right),(\pi, \mathcal{H}, P)$ is a classical system of imprimitivity, $\pi, P$ are both even, and $\overline{\rho^{\pi}(X)} \leftrightarrow P(E)$ for all $X \in \mathfrak{g}_{1}$ and Borel $E \subset \Omega$.

Let $\left(\pi, \rho^{\pi}, \mathcal{H}\right)$ be the induced representation defined in $\$ 3.2$ and let $P$ be the projection valued measure introduced above. Proposition $\mathbf{7}$ shows that $\left(\pi, \rho^{\pi}, \mathcal{H}, P\right)$ is a SSI based on $\Omega$. We call this the SSI induced by $\left(\sigma, \rho^{\sigma}\right)$.

Theorem 2 (super imprimitivity theorem) The assignment that takes $\left(\sigma, \rho^{\sigma}\right)$ to $\left(\pi, \rho^{\pi}, \mathcal{H}, P\right)$ is an equivalence of categories from the category of $\mathrm{UR}$ 's of the special sub SLG $\left(H_{0}, \mathfrak{h}\right)$ to the category of SSI 's based on $\Omega$.

Proof. Let us first prove that any SSI of the SLG $\left(G_{0}, \mathfrak{g}\right)$ is induced from a UR of the SLG $\left(H_{0}, \mathfrak{h}\right)$. We may assume, in view of the classical imprimitivity theorem that $\pi$ is the representation induced by a UR $\sigma$ of $H_{0}$ in $\mathcal{K}$ and that $\pi$ acts by left translations on $\mathcal{H}$. By assumption $\rho^{\pi}(X)$ leaves $C^{\infty}(\pi)$ invariant. We claim that it leaves $C_{c}^{\infty}(\pi)$ also invariant. Indeed, let $f \in C_{c}^{\infty}(\pi)$; then there is $u \in C_{c}^{\infty}(\Omega)$ such that $f=u f$. On the other hand, by Lemma 3. $\overline{\rho^{\pi}(X)} M(u)=M(u) \overline{\rho^{\pi}(X)}$ so that $u f \in D\left(\overline{\rho^{\pi}(X)}\right)$ and $\overline{\rho^{\pi}(X)}(u f)=$ $\overline{u \rho^{\pi}(X)} f$. Since $u f=f$ this comes to $\rho^{\pi}(X) f=u \rho^{\pi}(X) f$, showing that $\rho^{\pi}(X) f \in C_{c}^{\infty}(\pi)$. Thus the $\rho^{\pi}(X)$ leave $\mathcal{B}$ invariant and commute with all $M(u)$ there. In other words we may work with the smooth SSI. 
By Lemma 8 the map $f(1) \longmapsto\left(\rho^{\pi}(X) f\right)(1)$ is well defined and so, as in 3.1 we can define a map

$$
\rho^{\sigma}(X): C^{\infty}(\sigma) \longrightarrow C^{\infty}(\sigma)
$$

by

$$
\rho^{\sigma}(X) v=\left(\rho^{\pi}(X) f\right)(1), \quad f(1)=v, \quad f \in \mathcal{B} .
$$

Then, for $f \in \mathcal{B}, x \in G_{0}$,

$$
\begin{aligned}
\left(\rho^{\pi}(X) f\right)(x) & =\left(\pi\left(x^{-1}\right) \rho^{\pi}(X) f\right)(1) \\
& =\left(\rho^{\pi}\left(x^{-1} X\right) \pi\left(x^{-1}\right) f\right)(1) \\
& =\rho^{\sigma}\left(x^{-1} X\right)\left(\pi\left(x^{-1}\right) f\right)(1) \\
& =\rho^{\sigma}\left(x^{-1} X\right) f(x) .
\end{aligned}
$$

If we now prove that $\left(\sigma, \rho^{\sigma}, \mathcal{K}\right)$ is a UR of the $\operatorname{SLG}\left(H_{0}, \mathfrak{h}\right)$, we are done. This is completely formal.

Covariance with respect to $H_{0}$ : For $f \in \mathcal{B}, \xi \in H_{0}$,

$$
\begin{aligned}
\rho^{\sigma}(\xi X) f(1) & =\left(\rho^{\pi}(\xi X) f\right)(1) \\
& =\left(\pi(\xi) \rho^{\pi}(X) \pi\left(\xi^{-1}\right) f\right)(1) \\
& =\left(\rho^{\pi}(X) \pi\left(\xi^{-1}\right) f\right)\left(\xi^{-1}\right) \\
& =\sigma(\xi)\left(\rho^{\pi}(X) \pi\left(\xi^{-1}\right) f\right)(1) \\
& =\sigma(\xi) \rho^{\sigma}(X)\left(\pi\left(\xi^{-1}\right) f\right)(1) \\
& =\sigma(\xi) \rho^{\sigma}(X) \sigma(\xi)^{-1} f(1) .
\end{aligned}
$$

Odd commutators: Let $X, Y \in \mathfrak{g}_{1}=\mathfrak{h}_{1}$ so that $Z=[X, Y] \in \mathfrak{h}_{0}$. We have

$$
\left[\rho^{\pi}(X), \rho^{\pi}(Y)\right] f=-i d \pi([X . Y]) f
$$

for all $f \in \mathcal{B}$. Now,

$$
\begin{aligned}
i(-d \pi(Z) f)(1) & =i(d / d t)_{t=0} f(\exp t Z) \\
& =i(d / d t)_{t=0} \sigma(\exp (-t Z)) f(1) \\
& =-i d \sigma(Z) f(1) .
\end{aligned}
$$

On the other hand,

$$
\left(\rho^{\pi}(X) \rho^{\pi}(Y) f\right)(1)=\rho^{\sigma}(X) \rho^{\sigma}(Y) f(1)
$$

so that the left side of $(*)$, evaluated at 1 , becomes

$$
\left[\rho^{\sigma}(X), \rho^{\sigma}(Y)\right] f(1) .
$$


Thus

$$
\left[\rho^{\sigma}(X), \rho^{\sigma}(Y)\right] f(1)=-i d \sigma(Z) f(1) .
$$

Symmetry: From the symmetry of the $\rho^{\pi}(X)$ we have, for all $f, g \in$ $\mathcal{B}, a, b \in C_{c}^{\infty}(\Omega)$,

$$
\left(\rho^{\pi}(X)(a f), b g\right)_{\mathcal{H}}=\left(a f, \rho^{\pi}(X)(b g)\right)_{\mathcal{H}} .
$$

This means that

$$
\int\left(\rho^{\sigma}\left(x^{-1} X\right) f(x), g(x)\right)_{\mathcal{K}} a(x) \overline{b(x)} d \bar{x}=\int\left(f(x), \rho^{\sigma}\left(x^{-1} X\right) g(x)\right)_{\mathcal{K}} a(x) \overline{b(x)} d \bar{x} .
$$

Since $a$ and $b$ are arbitrary we conclude that

$$
\left(\rho^{\sigma}\left(x^{-1} X\right) f(x), g(x)\right)_{\mathcal{K}}=\left(f(x), \rho^{\sigma}\left(x^{-1} X\right) g(x)\right)_{\mathcal{K}}
$$

for almost all $x$. All functions in sight are continuous and so this relation is true for all $x$. The evaluation at 1 gives the symmetry of $\rho^{\sigma}(X)$ on $C^{\infty}(\sigma)$.

This proves that $\left(\sigma, \rho^{\sigma}, \mathcal{K}\right)$ is a UR of the $\operatorname{SLG}\left(H_{0}, \mathfrak{h}\right)$ and that the corresponding induced SSI is the one we started with.

To complete the proof we must show that the set of morphisms of the induced SSI's is in canonical bijection with the set of morphisms of the inducing UR's of the sub SLG in question. Let $\left(\pi, \rho^{\pi}, \mathcal{H}, P\right)$ and $\left(\pi^{\prime}, \rho^{\pi^{\prime}}, \mathcal{H}^{\prime}, P^{\prime}\right)$ be the SSI's induced by $\left(\sigma, \rho^{\sigma}\right)$ and $\left(\sigma^{\prime}, \rho^{\sigma^{\prime}}\right)$ respectively. For any morphism $R$ from $\left(\sigma, \rho^{\sigma}\right)$ to $\left(\sigma^{\prime}, \rho^{\sigma^{\prime}}\right)$ let $T$ be as in Theorem 1. Then $T$ extends uniquely to a bounded even operator from $\mathcal{H}$ to $\mathcal{H}^{\prime}$, and the relations $T M(u)=M^{\prime}(u) T$ for all $u \in C_{c}^{\infty}(\Omega)$ imply that $T P(E)=P^{\prime}(E) T$ for all Borel $E \subset \Omega$. Hence $T$ is a morphism from $\left(\pi, \rho^{\pi}, \mathcal{H}, P\right)$ to $\left(\pi^{\prime}, \rho^{\pi^{\prime}}, \mathcal{H}^{\prime}, P^{\prime}\right)$. It is clear that the assignment $R \longmapsto T$ is functorial. To complete the proof we must show that any morphism $T$ from $\left(\pi, \rho^{\pi}, \mathcal{H}, P\right)$ to $\left(\pi^{\prime}, \rho^{\pi^{\prime}}, \mathcal{H}^{\prime}, P^{\prime}\right)$ is of this form for a unique $R$. But $T$ must take $\mathcal{B}=C_{c}^{\infty}\left(\pi_{0}\right)$ to $\mathcal{B}^{\prime}=C_{c}^{\infty}\left(\pi_{0}^{\prime}\right)$ and commute with the actions of $C_{c}^{\infty}(\Omega)$. Hence $T$ is a morphism from $\left(\pi, \rho^{\pi}, \mathcal{B}, M\right)$ to $\left(\pi^{\prime}, \rho^{\pi^{\prime}}, \mathcal{B}^{\prime}, M^{\prime}\right)$. Theorem [1 now implies that $T$ arises from a unique morphism of $\left(\sigma, \rho^{\sigma}\right)$ to $\left(\sigma^{\prime}, \rho^{\sigma^{\prime}}\right)$.

This finishes the proof of Theorem 2 


\section{Representations of super semidirect prod- ucts and super Poincaré groups}

\subsection{Super semidirect products and their irreducible unitary representations}

We start with a classical semidirect product $G_{0}=T_{0} \times^{\prime} L_{0}$ where $T_{0}$ is a vector space of finite dimension over $\mathbb{R}$, the translation group, and $L_{0}$ is a closed unimodular subgroup of $\mathrm{GL}\left(T_{0}\right)$ acting on $T_{0}$ naturally. For any Lie group the corresponding gothic letter denotes its Lie algebra. In applications $L_{0}$ is usually an orthogonal group of Minkowskian signature, or its 2-fold cover, the corresponding spin group. By a super semidirect product (SSDP) we mean a SLG $\left(G_{0}, \mathfrak{g}\right)$ where $T_{0}$ acts trivially on $\mathfrak{g}_{1}$ and $\left[\mathfrak{g}_{1}, \mathfrak{g}_{1}\right] \subset \mathfrak{t}_{0}$. Clearly $\mathfrak{t}:=\mathfrak{t}_{0} \oplus \mathfrak{g}_{1}$ is also a super Lie algebra, and $\left(T_{0}, \mathfrak{t}\right)$ is a SLG called the super translation group. For any closed subgroup $S_{0} \subset L_{0}, H_{0}=T_{0} S_{0}$ is a closed subgroup of $G_{0}, \mathfrak{h}=\mathfrak{h}_{0} \oplus \mathfrak{g}_{1}$ is a super Lie algebra where $\mathfrak{h}_{0}=\mathfrak{t}_{0} \oplus \mathfrak{s}_{0}$ is the Lie algebra of $H_{0}$. Notice that $\left(H_{0}, \mathfrak{h}\right)$ is a special sub SLG of $\left(G_{0}, \mathfrak{g}\right)$. We begin by showing that the irreducible UR's of $\left(G_{0}, \mathfrak{g}\right)$ are in natural bijection with the irreducible UR's of suitable special sub SLG's of the form $\left(H_{0}, \mathfrak{h}\right)$ with the property that the translations act as scalars. For brevity we shall write $S=\left(G_{0}, \mathfrak{g}\right), T=\left(T_{0}, \mathfrak{t}\right)$.

The action of $L_{0}$ on $T_{0}$ induces an action on the dual $T_{0}^{*}$ of $T_{0}$. We assume that this action is regular, i.e., the orbits are all locally closed. By the well known theorem of Effros this implies that if $Q$ is any projection valued measure on $T_{0}^{*}$ such that $Q_{E}=0$ or the identity operator $I$ for any invariant Borel subset $E$ of $T_{0}^{*}$, then $Q$ is necessarily concentrated on a single orbit. This is precisely the condition under which the classical method of little groups of Frobenius-Mackey-Wigner works. For any $\lambda \in T_{0}^{*}$ let $L_{0}^{\lambda}$ be the stabilizer of $\lambda$ in $L_{0}$ and let $\mathfrak{g}^{\lambda}=\mathfrak{t}_{0} \oplus \mathfrak{l}_{0}^{\lambda} \oplus \mathfrak{g}_{1}$. The SLG $\left(T_{0} L_{0}^{\lambda}, \mathfrak{g}^{\lambda}\right)$ will be denoted by $S^{\lambda}$. We shall call it the little super group at $\lambda$. It is a special sub SLG of $\left(G_{0}, \mathfrak{g}\right)$. Two $\lambda$ 's are called equivalent if they are in the same $L_{0}$-orbit. If $\theta$ is a UR of the classical group $T_{0} L_{0}$ and $O$ is an orbit in $T_{0}^{*}$, its spectrum is said to be in $O$ if the spectral measure (via the SNAG theorem) of the restriction of $\theta$ to $T_{0}$ is supported by $O$.

Given $\lambda \in T_{0}^{*}$, a UR $\left(\sigma, \rho^{\sigma}\right)$ of $S^{\lambda}$ is $\lambda$-admissible if $\sigma(t)=e^{i \lambda(t)} I$ for $t \in T_{0} . \quad \lambda$ itself is called admissible if there is an irreducible UR which is $\lambda$-admissible. It is obvious that the property of being admissible is preserved 
under the action of $L_{0}$. Let

$$
T_{0}^{+}=\left\{\begin{array}{l|l}
\lambda \in T_{0}^{*} & \lambda \text { admissible }\} .
\end{array}\right.
$$

Then $T_{0}^{+}$is an invariant subset of $T_{0}^{*}$.

Theorem 3 The spectrum of every irreducible UR of the SLG $\left(G_{0}, \mathfrak{g}\right)$ is in some orbit in $T_{0}^{+}$. For each orbit in $T_{0}^{+}$and choice of $\lambda$ in that orbit, the assignment that takes a $\lambda$-admissible UR $\gamma:=\left(\sigma, \rho^{\sigma}\right)$ of $S^{\lambda}$ into the UR $U^{\gamma}$ of $\left(G_{0}, \mathfrak{g}\right)$ induced by it, is a functor which is an equivalence of categories between the category of the $\lambda$-admissible UR's of $S^{\lambda}$ and the category of UR's of $\left(G_{0}, \mathfrak{g}\right)$ with their spectra in that orbit. Varying $\lambda$ in that orbit changes the functor into an equivalent one. In particular this functor gives a bijection between the respective sets of equivalence classes of irreducible UR's.

Proof. Notice first of all that since $T_{0}$ acts trivially on $\mathfrak{g}_{1}, \pi_{0}(t)$ commutes with $\rho^{\pi}(X)$ on $C^{\infty}\left(\pi_{0}\right)$ for all $t \in T_{0}, X \in \mathfrak{g}_{1}$. Hence $P_{E} \leftrightarrow \rho^{\pi}(X)$ for all Borel $E \subset \Omega, X \in \mathfrak{g}_{1}$. For the first statement, let $E$ be an invariant Borel subset of $T_{0}^{*}$. Let $P$ be the spectral measure of the restriction of $\pi$ to $T_{0}$. Then $P_{E}$ commutes with $\pi, \rho^{\pi}(X)$. So, if $\left(\pi, \rho^{\pi}\right)$ is irreducible, $P_{E}=0$ or $I$. Hence $P$ is concentrated in some orbit $O$, i.e., $P_{O}=I$. The system $\left(\pi, \rho^{\pi}\right)$ is clearly equivalent to $\left(\pi, \rho^{\pi}, P\right)$ since $P$ and the restriction of $\pi$ to $T_{0}$ generate the same algebra. If $\lambda \in O$ and $L_{0}^{\lambda}$ is the stabilizer of $\lambda$ in $L_{0}$, we can transfer $P$ from $O$ to a projection valued measure $P^{*}$ on $L_{0} / L_{0}^{\lambda}=T_{0} L_{0} / T_{0} L_{0}^{\lambda}$. So $\left(\pi, \rho^{\pi}\right)$ is equivalent to the SSI $\left(\pi, \rho^{\pi}, P^{*}\right)$. The rest of the theorem is an immediate consequence of Theorem 2. The fact that $\sigma(t)=e^{i \lambda(t)} I$ for $t \in T_{0}$ is classical. Indeed, in the smooth model for $\pi$ treated in $\$ 3.2$, the fact that the spectrum of $\pi$ is contained in the orbit of $\lambda$ implies that $(\pi(t) f)(x)=e^{i \lambda\left(x^{-1} t x\right)} f(x)$ for all $f \in \mathcal{B}, t \in T_{0}, x \in G_{0}$. Hence $f\left(t^{-1}\right)=e^{i \lambda(t)} f(1)$ while $f\left(t^{-1}\right)=\sigma(t) f(1)$. So $\sigma(t)=e^{i \lambda(t)} I$.

Remark 2 In the classical theory all orbits of $L_{0}$ are allowed and an additional argument of the positivity of energy is needed to single out the physically occurring representations. In SUSY theories as exemplified by Theorem 3 , a restriction is already present: only orbits in $T_{0}^{+}$are permitted. We shall prove in the next section that $T_{0}^{+}$may be interpreted precisely as the set of all positive energy representations. 


\subsection{Determination of the admissible orbits. Product structure of the representations of the little super groups}

We fix $\lambda \in T_{0}^{+}$and let $\left(\sigma, \rho^{\sigma}\right)$ be a $\lambda$-admissible irreducible UR of $S^{\lambda}$. Clearly

$$
-i d \sigma(Z)=\lambda(Z) I \quad\left(Z \in \mathfrak{t}_{0}\right) .
$$

Define

$$
\Phi_{\lambda}\left(X_{1}, X_{2}\right)=(1 / 2) \lambda\left(\left[X_{1}, X_{2}\right]\right) \quad\left(X_{1}, X_{2} \in \mathfrak{g}_{1}\right) .
$$

Then, on $C^{\infty}(\sigma)$,

$$
\left[\rho^{\sigma}\left(X_{1}\right), \rho^{\sigma}\left(X_{2}\right)\right]=\lambda\left(\left[X_{1}, X_{2}\right]\right) I=2 \Phi_{\lambda}\left(X_{1}, X_{2}\right) I
$$

Clearly $\Phi_{\lambda}$ is a symmetric bilinear form on $\mathfrak{g}_{1} \times \mathfrak{g}_{1}$. Let

$$
Q_{\lambda}(X)=\Phi_{\lambda}(X, X)=(1 / 2) \lambda([X, X]) .
$$

Then $Q_{\lambda}$ is invariant under $L_{0}^{\lambda}$ because for $X_{1}, X_{2} \in \mathfrak{g}_{1}, h \in L_{1}$,

$$
\left[\rho^{\sigma}\left(h X_{1}\right), \rho^{\sigma}\left(h X_{2}\right)\right]=\sigma(h)\left[\rho^{\sigma}\left(X_{1}\right), \rho^{\sigma}\left(X_{2}\right)\right] \sigma(h)^{-1}=2 \Phi_{\lambda}\left(X_{1}, X_{2}\right) .
$$

Now

$$
\rho^{\sigma}(X)^{2}=Q_{\lambda}(X) I \quad\left(X \in \mathfrak{g}_{1}\right) .
$$

Since $\rho^{\sigma}(X)$ is essentially self adjoint on $C^{\infty}(\sigma)$, it is immediate that $Q_{\lambda}(X) \geq$ 0 . We thus obtain the necessary condition for admissibility:

$$
Q_{\lambda}(X)=\Phi_{\lambda}(X, X) \geq 0 \quad\left(X \in \mathfrak{g}_{1}\right) .
$$

In the remainder of this subsection we shall show that the condition that $\Phi_{\lambda} \geq 0$, which we refer to as the positive energy condition, is also sufficient to ensure that $\lambda$ is admissible. We will then find all the $\lambda$-admissible irreducible UR's of $S^{\lambda}$.

It will follow in the next section that if the super Lie group $\left(G_{0}, \mathfrak{g}_{1}\right)$ is a super Poincaré group, the condition $\Phi_{\lambda} \geq 0$ expresses precisely the positivity of the energy. This is the reason for our describing this condition in the general case also as the positive energy condition.

From now on we fix $\lambda$ such that $\Phi_{\lambda} \geq 0$.

Lemma 10 For any admissible UR $\left(\sigma, \rho^{\sigma}\right)$ of $S^{\lambda}, \rho^{\sigma}(X)$ is a bounded self adjoint operator for $X \in \mathfrak{g}_{1}$, and $\rho^{\sigma}(X)^{2}=Q_{\lambda}(X) I$. Moreover, $Q_{\lambda} \geq 0$ and is invariant under $L_{0}^{\lambda}$. 
Proof. We have, for $X \in \mathfrak{g}_{1}, \psi \in C^{\infty}(\sigma)$,

$$
\left|\rho^{\sigma}(X) \psi\right|_{\mathcal{K}}^{2}=\left(\rho^{\sigma}(X)^{2} \psi, \psi\right)=Q_{\lambda}(X)^{2}|\psi|_{\mathcal{K}}^{2}
$$

which proves the lemma.

This lemma suggests we study the following situation. Let $W$ be a finite dimensional real vector space and let $q$ be a nonnegative quadratic form on $W$, i.e., $q(w) \geq 0$ for $w \in W$. Let $\varphi$ be the corresponding symmetric bilinear form $(q(w)=\varphi(w, w))$. Let $\mathcal{C}$ be the real algebra generated by $W$ with the relations $w^{2}=q(w) 1(w \in W)$. If $q$ is nondegenerate, i.e., positive definite, this is the Clifford algebra associated to the quadratic vector space $(W, q)$. If $q=0$ it is just the exterior algebra over $W$. If $\left(w_{i}\right)_{1 \leq i \leq n}$ is a basis for $W$ such that $\varphi\left(w_{i}, w_{j}\right)=\varepsilon_{i} \delta_{i j}$ with $\varepsilon_{i}=0$ or 1 according as $i \leq a$ or $>a$, then $\mathcal{C}$ is the algebra generated by the $w_{i}$ with the relations $w_{i} w_{j}+w_{j} w_{i}=2 \varepsilon_{i} \delta_{i j}$. Let $W_{0}$ be the radical of $q$, i.e., $W_{0}=\left\{w_{0} \mid \varphi\left(w_{0}, w\right)=0\right.$ for all $\left.w \in W\right\}$; in the above notation $W_{0}$ is spanned by the $w_{i}$ for $i \leq a$. If $W^{\sim}=W / W_{0}$ and $q^{\sim}, \varphi^{\sim}$ are the corresponding objects induced on $W^{\sim}, q^{\sim}$ is positive definite, and so we have the usual Clifford algebra $\mathcal{C}^{\sim}$ generated by $\left(W^{\sim}, q^{\sim}\right)$ with $W^{\sim} \subset \mathcal{C}^{\sim}$. The natural map $W \longrightarrow W^{\sim}$ extends uniquely to a morphism $\mathcal{C} \longrightarrow \mathcal{C}^{\sim}$ which is clearly surjective. We claim that its kernel is the ideal $\mathcal{C}_{0}$ in $\mathcal{C}$ generated by $W_{0}$. Indeed, let $I$ be this kernel. If $s \in I, s$ is a linear combination of elements $w_{I} w_{J}$ where $w_{I}$ is a product $w_{i_{1}} \ldots w_{i_{r}}\left(i_{1}<\right.$ $\left.\cdots<i_{r} \leq a\right)$ and $w_{J}$ is a product $w_{j_{1}} \ldots w_{j_{s}}\left(a<j_{1}<\cdots<j_{s}\right)$; hence, $s \equiv \sum_{J} c_{J} w_{J} \bmod \mathcal{C}_{0}$, and as the image of this element in $\mathcal{C}^{\sim}$ is $0, c_{J}=0$ for all $J$ because the images of the $w_{J}$ are linearly independent in $\mathcal{C}^{\sim}$. Hence $s \in \mathcal{C}_{0}$, proving our claim.

A representation $\theta$ of $\mathcal{C}$ by bounded operators in a SHS $\mathcal{K}$ is called self adjoint (SA) if $\theta(w)$ is odd and self adjoint for all $w \in W$. $\theta$ can be viewed as a representation of the complexification $\mathbb{C} \otimes \mathcal{C}$ of $\mathcal{C}$; a representation of $\mathbb{C} \otimes \mathcal{C}$ arises in this manner from a $\mathrm{SA}$ representation of $\mathcal{C}$ if and only if it maps elements of $W$ into odd operators and takes complex conjugates to adjoints. Also we wish to stress that irreducibility is in the graded sense.

Lemma 11 ( $i$ ) If $\tau$ is a $\mathrm{SA}$ representation of $\mathcal{C}$ in $\mathcal{K}$, then $\tau=0$ on $\mathcal{C}_{0}$ and so it is the lift of a $\mathrm{SA}$ representation $\tau^{\sim}$ of $\mathcal{C}^{\sim}$. (ii) There exist irreducible $\mathrm{SA}$ representations $\tau$ of $\mathcal{C}$; these are finite dimensional, unique if $\operatorname{dim}\left(W^{\sim}\right)$ is odd, and unique up to parity reversal if $\operatorname{dim}\left(W^{\sim}\right)$ is even. (iii) Let $\tau$ be an irreducible $\mathrm{SA}$ representation of $\mathcal{C}$ in a SHS $\mathcal{L}$ and let $\theta$ be any $\mathrm{SA}$ representation of $\mathcal{C}$ in a SHS $\mathcal{R}$. Then $\mathcal{R} \simeq \mathcal{K} \otimes \mathcal{L}$ where $\mathcal{K}$ is a SHS and $\theta(a)=1 \otimes \tau(a)$ for all $a \in \mathcal{C}$; moreover, if $\operatorname{dim}\left(W^{\sim}\right)$ is odd, we can choose $\mathcal{K}$ to be purely even. 
Proof. (i) If $w \in W_{0}$, then $\tau\left(w_{0}\right)^{2}=q\left(w_{0}\right) I=0$ and so, $\tau\left(w_{0}\right)$ itself must be 0 since it is self adjoint.

(ii) In view of (i) we may assume that $W_{0}=0$ so that $q$ is positive definite. Case $I: \operatorname{dim}(W)=2 m$. Select an ON basis $a_{1}, b_{1}, \ldots, a_{m}, b_{m}$ for $W$. Let $e_{j}=(1 / 2)\left(a_{j}+i b_{j}\right), f_{j}=(1 / 2)\left(a_{j}-i b_{j}\right)$. Then $\varphi\left(e_{j}, e_{k}\right)=\varphi\left(f_{j}, f_{k}\right)=0$ while $\varphi\left(e_{j}, f_{k}\right)=(1 / 2) \delta_{j k}$. Then $\mathbb{C} \otimes \mathcal{C}$ is generated by the $e_{j}, f_{k}$ with the relations

$$
e_{j} e_{k}+e_{k} e_{j}=f_{j} f_{k}+f_{k} f_{j}=0, \quad e_{j} f_{k}+f_{k} e_{j}=\delta_{j k} .
$$

We now set up the standard "Schrödinger" representation of $\mathbb{C} \otimes \mathcal{C}$. The representation acts on the SHS $\mathcal{L}=\Lambda(U)$ where $U$ is a Hilbert space of dimension $m$ and the grading on $\mathcal{L}$ is the $\mathbb{Z}_{2}$-grading induced by the usual $\mathbb{Z}$-grading of $\Lambda(U)$. Let $\left(u_{j}\right)_{1 \leq j \leq m}$ be an ON basis for $U$. We define

$$
\tau\left(e_{j}\right) f=u_{j} \wedge f, \quad \tau\left(f_{j}\right) f=\partial\left(u_{j}\right)(f) \quad(f \in \Lambda(U)),
$$

$\partial(u)$ for any $u \in U$ being the odd derivation on $\Lambda(U)$ such that $\partial(u) v=$ $2(v, u)$ (here $(\cdot, \cdot)$ is the scalar product in $\Lambda(U)$ extending the scalar product of $U$ ). It is standard that $\tau$ is an irreducible representation of $\mathbb{C} \otimes \mathcal{C}$. The vector 1 is called the Clifford vacuum. We shall now verify that $\tau$ is SA Since $a_{j}=e_{j}+f_{j}, b_{j}=-i\left(e_{j}-f_{j}\right)$, we need to verify that $\tau\left(f_{j}\right)=\tau\left(e_{j}\right)^{*}$ for all $j$, * denoting adjoints. For any subset $K=\left\{k_{1}<\cdots<k_{r}\right\} \subset\{1,2, \ldots, m\}$ we write $u_{K}=u_{k_{1}} \wedge \cdots \wedge u_{k_{r}}$. Then we should verify that

$$
\left(u_{j} \wedge u_{K}, u_{L}\right)=\left(u_{K}, \partial\left(u_{j}\right) u_{L}\right) \quad(K, L \subset\{1,2, \ldots, m\}) .
$$

Write $K=\left\{k_{1}, \ldots, k_{r}\right\}, L=\left\{\ell_{1}, \ldots, \ell_{s}\right\}$ where $k_{1}<\cdots<k_{r}, \ell_{1}<\cdots<\ell_{s}$. We assume that $j=\ell_{a}$ for some $a$ and $K=L \backslash\left\{\ell_{a}\right\}$, as otherwise both sides are 0 . Then $K=\left\{\ell_{1}, \ldots, \ell_{a-1}, \ell_{a+1}, \ldots, \ell_{s}\right\}$ (note that $r=s-1$ ). But then both sides are equal to $(-1)^{a-1}$.

From the general theory of Clifford algebras we know that if $\tau^{\prime}$ is another irreducible SA representation of $\mathcal{C}$, then either $\tau \approx \tau^{\prime}$ or else $\tau \approx \Pi \tau^{\prime} \Pi$ where $\Pi$ is the parity revarsal map and we write $\approx$ for linear (not necessarily unitary) equivalence. So it remains to show that $\approx$ implies unitary equivalence which we write $\simeq$. This is standard since the linear equivalence preserves self adjointness. Indeed, if $R: \tau_{1} \longrightarrow \tau_{2}$ is an even linear isomorphism, then $R^{*} R$ is an even automorphism of $\tau_{1}$ and so $R^{*} R=a^{2} I$ where $a$ is a scalar which is $>0$. Then $U=a^{-1} R$ is an even unitary isomorphism $\tau_{1} \simeq \tau_{2}$. Also for use in the odd case to be treated next, we note that $\tau$ is irreducible in the ungraded sense since its image is the full endomorphism algebra of $\mathcal{L}$. 
Case $I I: \operatorname{dim}(W)=2 m+1$. It is enough to construct an irreducible SA representation as it will be unique up to linear, and hence unitary, equivalence.

Let $a_{0}, a_{1}, \ldots, a_{2 m}$ be an ON basis for $W$. Write $x_{j}=i a_{0} a_{j}(1 \leq j \leq$ $2 m), \quad x_{0}=i^{m} a_{0} a_{1} \ldots a_{2 m}$. Then $x_{0}^{2}=1, x_{j} x_{k}+x_{k} x_{j}=2 \delta_{j k}(j, k=1,2, \ldots, 2 m)$. Moreover $x_{0}$ commutes with all $a_{j}$ and hence with all $x_{j}$. The $x_{j}$ generate a Clifford algebra over $\mathbb{R}$ corresponding to a positive definite quadratic form and so there is an irreducible ungraded representation $\tau^{+}$of it in an ungraded Hilbert space $\mathcal{L}^{+}$such that $\tau^{+}\left(x_{j}\right)$ is self adjoint for all $j=1,2, \ldots, 2 m$ (cf. remark above). Within $\mathbb{C} \otimes \mathcal{C}$ the $x_{j}$ generate $\mathbb{C} \otimes \mathcal{C}^{+}$so that $\tau^{+}$is a representation of $\mathbb{C} \otimes \mathcal{C}^{+}$in $\mathcal{L}^{+}$such that $i \tau^{+}\left(a_{0} a_{j}\right)$ is self adjoint for all $j$. We now take

$$
\mathcal{L}=\mathcal{L}^{+} \oplus \mathcal{L}^{+}, \quad \tau=\tau^{+} \oplus \tau^{+}, \quad \tau\left(x_{0}\right)=\left(\begin{array}{ll}
0 & 1 \\
1 & 0
\end{array}\right)
$$

Here $\mathcal{L}$ is given the $\mathbb{Z}_{2}$-grading such that the first and second copies of $\mathcal{L}^{+}$ are the even and odd parts. It is clear that $\tau$ is an irreducible representation of $\mathbb{C} \otimes \mathcal{C}$. We wish to show that $\tau\left(a_{r}\right)$ is odd and self adjoint for $0 \leq r \leq 2 m$. But this follows from the fact that the $\tau\left(x_{r}\right)$ are self adjoint, $\tau\left(x_{j}\right)$ are even, and $\tau\left(x_{0}\right)$ is odd, in view of the formulae

$$
a_{0}=i^{m} x_{0} x_{1} \ldots x_{2 m}, \quad a_{j}=-i a_{0} x_{j} .
$$

This finishes the proof of (ii).

(iii) Let now $\theta$ be a SA representation of $\mathcal{C}$ in a SHS $\mathcal{R}$ of possibly infinite dimension. For any homogeneous $\psi \in \mathcal{R}$ the cyclic subspace $\theta(\mathcal{C}) \psi$ is finite dimensional, hence closed, graded and is $\theta$-stable; moreover by the SA nature of $\theta$, for any graded invariant subspace its orthogonal complement is also graded and invariant. Hence we can write $\mathcal{R}=\oplus_{\alpha} \mathcal{R}_{\alpha}$ where the sum is direct and each $\mathcal{R}_{\alpha}$ is graded, invariant, and irreducible. Let $\mathcal{L}$ be a SHS on which we have an irreducible SA representation of $\mathcal{C}$. If $\operatorname{dim}(W)^{\sim}$ is even we can thus write $\mathcal{R}=\left(\mathcal{M}_{0} \otimes \mathcal{L}\right) \oplus\left(\mathcal{M}_{1} \otimes \Pi \mathcal{L}\right)$ where the $\mathcal{M}_{j}$ are even Hilbert spaces; if $\operatorname{dim}\left(W^{\sim}\right)$ is odd we can write $\mathcal{R}=\mathcal{K} \otimes \mathcal{L}$ where $\mathcal{K}$ is an even Hilbert space. In the first case, since $\mathcal{M}_{1} \otimes \Pi \mathcal{L}=\Pi \mathcal{M}_{1} \otimes \mathcal{L}$, we have $\mathcal{R}=\mathcal{K} \otimes \mathcal{L}$ where $\mathcal{K}$ is a SHS with $\mathcal{K}_{0}=\mathcal{M}_{0}, \mathcal{K}_{1}=\Pi \mathcal{M}_{1}$.

For studying the question of admissibility of $\lambda$ we need a second ingredient. Let $H$ be a not necessarily connected Lie group and let us be given a morphism

$$
j: H \longrightarrow \mathrm{O}\left(W^{\sim}\right)
$$

so that $H$ acts on $W^{\sim}$ preserving the quadratic form on $W^{\sim}$. We wish to find out when there is a UR $\kappa$ of $H$, possibly projective, and preferably, but 
not necessarily, even, in the space of the irreducible SA representation $\tau^{\sim}$, such that

$$
\kappa(t) \tau^{\sim}(w) \kappa(t)^{-1}=\tau^{\sim}(t w) \quad\left(t \in H, w \in W^{\sim}\right)
$$

For $h \in \mathrm{O}\left(W^{\sim}\right)$, let

$$
\tau_{h}^{\sim}(w)=\tau^{\sim}(h w) \quad\left(w \in W^{\sim}\right) .
$$

Then $\tau_{h}^{\sim}$ is also an irreducible SA representation of $\mathcal{C}^{\sim}$ and so we can find a unitary operator $K(h)$ such that

$$
\tau_{h}^{\sim}(w)=K(h) \tau^{\sim}(w) K(h)^{-1} \quad\left(w \in W^{\sim}\right) .
$$

If $\operatorname{dim} W^{\sim}$ is even, $\tau^{\sim}$ is irreducible even as an ungraded representation, and so $K(h)$ will be unique up to a phase; it will be even or odd according as $\tau_{h}^{\sim} \simeq \tau^{\sim}$ or $\tau_{h}^{\sim} \simeq \Pi \tau^{\sim} \Pi$ where $\Pi$ is parity reversal. If $\operatorname{dim} W^{\sim}$ is odd, $\tau^{\sim}$ is irreducible only as a graded representation and so we also need to require $K(h)$ to be an even operator in order that it is uniquely determined up to a phase. With this additional requirement in the odd dimensional case, we then see that in both cases the class of $\kappa$ as a projective UR of $H$ is uniquely determined, i.e., the class of its multiplier $\mu$ in $H^{2}(H, \mathbb{T})$ is fixed. In the following, we shall show that $\mu$ can be chosen to be \pm 1 -valued, and examine the structure of $\kappa$ more closely.

We begin with some preparation (see [Del99, group of invertible elements in $\mathcal{C}^{\sim}$. Define the full Clifford group as follows:

$$
\Gamma=\left\{x \in \mathcal{C}^{\sim \times} \cap\left(\mathcal{C}^{\sim+} \cup \mathcal{C}^{\sim-}\right) \mid x W^{\sim} x^{-1} \subset W^{\sim}\right\} .
$$

We have a homomorphism $\alpha: \Gamma \longrightarrow \mathrm{O}\left(\mathrm{W}^{\sim}\right)$ given by

$$
\alpha(x) w=(-1)^{p(x)} x w x^{-1}
$$

for all $w \in W^{\sim}, p(x)$ being 0 or 1 according as $x \in \mathcal{C}^{\sim+}$ or $x \in \mathcal{C}^{\sim-}$. Let $\beta$ be the principal antiautomorphism of $\mathcal{C}^{\sim+}$; then $x \beta(x) \in \mathbb{R}^{\times}$for all $x \in \Gamma$, and we write $G$ for the kernel of the homomorphism $x \mapsto x \beta(x)$ of $\Gamma$ into $\mathbb{R}^{\times}$. Since $W^{\sim}$ is a positive definite quadratic space, we have an exact sequence

$$
1 \longrightarrow\{ \pm 1\} \longrightarrow G \stackrel{\alpha}{\longrightarrow} \mathrm{O}\left(W^{\sim}\right) \longrightarrow 1 .
$$

For $\operatorname{dim} W^{\sim} \geq 2$, the connected component $G^{0}$ of $G$ is contained in $\mathcal{C}^{\sim+}$ and coincides with $\operatorname{Spin}\left(W^{\sim}\right)$.

Lemma 12 Let $\tau^{\sim}$ be a SA irreducible representation of $\mathcal{C}^{\sim}$. We then have the following. (i) $\tau^{\sim}$ restricts to a unitary representation of $G$. (ii) The operator $\tau^{\sim}(x)$ is even or odd according as $x \in G^{0}$ or $x \in G \backslash G^{0}$. (iii) $\tau^{\sim}(x) \tau^{\sim}(w) \tau^{\sim}(x)^{-1}=(-1)^{p(x)} \tau^{\sim}(\alpha(x)(w))$ for $x \in G, w \in W^{\sim}$. 
Proof. Each $x \in G$ is expressible in the form $x=c v_{1} \ldots v_{r}$, where $v_{i}$ are unit vectors in $W^{\sim}$ and $c \in\{ \pm 1\}$. Since $\tau^{\sim}\left(v_{i}\right)$ is odd, the parity of $\tau^{\sim}(x)$ is the same of $x$. Moreover, since the $\tau^{\sim}\left(v_{i}\right)$ are self adjoint,

$$
\tau^{\sim}(x) \tau^{\sim}(x)^{*}=c^{2} \tau^{\sim}\left(v_{1}\right) \ldots \tau^{\sim}\left(v_{r}\right) \tau^{\sim}\left(v_{r}\right) \ldots \tau^{\sim}\left(v_{1}\right)=I .
$$

This proves (i). (ii) and (iii) are obvious. - We now consider two cases.

Case I. $j(H) \subset \mathrm{SO}\left(W^{\sim}\right)$.

Let $\zeta$ be a Borel map of $\mathrm{SO}\left(W^{\sim}\right)$ into $\operatorname{Spin}\left(W^{\sim}\right)$ which is a right inverse of $\alpha\left(\operatorname{Spin}\left(W^{\sim}\right) \longrightarrow \mathrm{SO}\left(W^{\sim}\right)\right)$ with $\zeta(1)=1$. Then $\zeta(x y)= \pm \zeta(x) \zeta(y)$ for $x, y \in \mathrm{SO}\left(W^{\sim}\right)$, and so

$$
\kappa_{H}=\tau^{\sim} \circ \zeta \circ j
$$

is an even projective UR of $H$ satisfying $(*)$ with a \pm -valued multiplier $\mu_{H}$. Since $\zeta(1)=1$ it follows that $\mu_{H}$ is normalized, i.e.,

$$
\mu_{H}(h, 1)=\mu_{H}(1, h)=1 \quad(h \in H) .
$$

Clearly, the class of $\mu_{H}$ in $H^{2}\left(H, \mathbb{Z}_{2}\right)$ is trivial if and only if $j: H \rightarrow \mathrm{SO}\left(W^{\sim}\right)$ can be lifted to a morphism $\widehat{j}: H \rightarrow \operatorname{Spin}\left(W^{\sim}\right)$. In particular, this happens if $H$ is connected and simply connected.

Suppose now $H$ is connected but $\widehat{j}$ does not exist. We can then find a two-fold cover $H^{\sim}$ of $H$ with a covering map $p\left(H^{\sim} \longrightarrow H\right)$ such that $j\left(H \rightarrow \mathrm{SO}\left(W^{\sim}\right)\right)$ lifts to a morphism $j^{\sim}\left(H^{\sim} \rightarrow \operatorname{Spin}\left(W^{\sim}\right)\right)$, and if $\xi$ is the nontrivial element in $\operatorname{ker} p$, then $j^{\sim}(\xi)=-1$.

Lemma 13 If $j$ maps $H$ into $\mathrm{SO}\left(W^{\sim}\right)$, there is a projectively unique even projective $\mathrm{UR} \kappa$ of $H$ satisfying $(*)$, with a normalized \pm 1 -valued multiplier $\mu$. If $H$ is connected, for $\kappa$ to be an ordinary even representation (which will be unique up to multiplication by a character of $H$ ) it is necessary and sufficient that either (i) $j\left(H \rightarrow \mathrm{SO}\left(W^{\sim}\right)\right)$ can be lifted to $\operatorname{Spin}\left(W^{\sim}\right)$ or (ii) there exists a character $\chi$ of $H^{\sim}$ such that $\chi(\xi)=-1$. In particular, if $H=A \times^{\prime} T$, where $A$ is simply connected and $T$ is a torus, then $\kappa$ is an ordinary even unitary representation.

Proof. The first statement has already been proved.

We next prove the sufficiency part of the second statement. Sufficiency of (i) has already been observed. To see that (ii) is sufficient, note that $\kappa^{\sim}=\tau^{\sim} \circ j^{\sim}$ is an even UR of $H^{\sim}$ satisfying $(*)$; one can clearly replace $\kappa^{\sim}$ by $\kappa^{\sim} \chi$ without destroying $(*)$. As $\kappa^{\sim}(\xi)=-1$, we have $\left(\kappa^{\sim} \chi\right)(\xi)=1$, and so it is immediate that $\kappa^{\sim} \chi$ descends to $H$.

We leave the necessity part to the reader; it will not be used in the sequel. 
The statement for $H=A \times^{\prime} T$ will follow if we show that $H^{\sim}$ has a character $\chi$ as in condition (ii). We have $H^{\sim}=A \times^{\prime} T^{\sim}, T^{\sim}$ being the double cover of $T$, and $\xi=(1, t)$, with $t \neq 1, t^{2}=1$. There exists a character $\chi$ of $T^{\sim}$ such that $\chi(t)=-1$, and such a character can be extended to $H^{\sim}$ by making it trivial on $A$.

Case II. $j(H) \not \subset \mathrm{SO}\left(W^{\sim}\right)$.

Let

$$
H_{0}=j^{-1}\left(\mathrm{SO}\left(W^{\sim}\right)\right) .
$$

Then $H_{0}$ is a normal subgroup of $H$ of index 2. We must distinguish two subcases.

Case II.a. $\operatorname{dim}\left(W^{\sim}\right)$ is even.

Let $\zeta_{0}$ be a Borel right inverse of $\alpha\left(G^{0} \longrightarrow \mathrm{SO}\left(W^{\sim}\right)\right)$ with $\zeta_{0}(1)=1$. Fix a unit vector $v_{0} \in W^{\sim}$ and let $r_{0}=-\alpha\left(v_{0}\right)$. Since $\alpha\left(v_{0}\right)$ is the reflection in the hyperplane orthogonal to $v_{0}$ and $\operatorname{dim}\left(W^{\sim}\right)$ is even, we see that $r_{0} \in$ $\mathrm{O}\left(W^{\sim}\right) \backslash \mathrm{SO}\left(W^{\sim}\right)$. We then define a map $\zeta\left(\mathrm{O}\left(W^{\sim}\right) \rightarrow G\right)$ by

$$
\zeta(h)= \begin{cases}\zeta_{0}(h) & \text { if } h \in \mathrm{SO}\left(W^{\sim}\right) \\ \zeta_{0}\left(h_{0}\right) v_{0} & \text { if } h=h_{0} r_{0}, h_{0} \in \mathrm{SO}\left(W^{\sim}\right) .\end{cases}
$$

Once again we have $\zeta\left(h_{1} h_{2}\right)= \pm \zeta\left(h_{1}\right) \zeta\left(h_{2}\right)$. Define

$$
\kappa_{H}=\tau^{\sim} \circ \zeta \circ j
$$

Then $\kappa_{H}$ is a projective UR of $H$ satisfying $(*)$ with a \pm -valued normalized multiplier $\mu_{H}$. But $\kappa_{H}$ is not an even representation; elements of $H \backslash H_{0}$ map into odd unitary operators in the space of $\tau^{\sim}$. We shall call such a representation of $H$ graded with respect to $H_{0}$, or simply graded. We have thus proved the following.

Lemma 14 If $j(H) \not \subset \mathrm{SO}\left(W^{\sim}\right)$, and $\operatorname{dim}\left(W^{\sim}\right)$ is even, and if we define $H_{0}=j^{-1}\left(\mathrm{SO}\left(W^{\sim}\right)\right)$, then there is a projective $\mathrm{UR} \kappa_{H}$, graded with respect to $H_{0}$, and satisfying $(*)$ with a \pm -valued normalized multiplier $\mu_{H}$.

Before we take up the case when $\operatorname{dim}\left(W^{\sim}\right)$ is odd, we shall describe how the projective graded representations of $H$ are constructed. This is a very general situation and so we shall work with a locally compact second countable group $A$ and a closed subgroup $A_{0}$ of index $2 ; A_{0}$ is automatically normal and we write $A_{1}=A \backslash A_{0}$. Gradedness is with respect to $A_{0}$. We fix a \pm -valued multiplier $\mu$ for $A$ which is normalized. For brevity a representation will mean a unitary $\mu$-representation. Moreover, with a slight abuse of language a $\mu$-representation of $A_{0}$ will mean a $\left.\mu\right|_{A_{0} \times A_{0}}$-representation of 
$A_{0}$. If $R_{g}$ is a graded representation in a SHS $\mathcal{H}, R$ the corresponding ungraded representation, and $P_{j}$ is the orthogonal projection $\mathcal{H} \longrightarrow \mathcal{H}_{j}$, we associate to $R$ the projection valued measure $P$ on $A / A_{0}$ where $P_{A_{0}}=P_{0}$ and $P_{A_{1}}=P_{1}$. Then the condition that $R_{g}$ is graded is exactly the same as saying that $(R, P)$ is a system of imprimitivity for $A$ based on $A / A_{0}$. Conversely, given a system of imprimitivity $(R, P)$ for $A$ based on $A / A_{0}$, let us define the grading for $\mathcal{H}=\mathcal{H}(R)$ by $\mathcal{H}_{j}=$ range of $P_{A_{j}}(j=0,1)$; then $R$ becomes a graded representation. Moreover for graded representations $R_{g}, R_{g}^{\prime}$, we have $\operatorname{Hom}\left(R_{g}, R_{g}^{\prime}\right)=\operatorname{Hom}\left((R, P),\left(R^{\prime}, P^{\prime}\right)\right)$. In other words, the category of systems of imprimitivity for $A$ based on $A / A_{0}$ and the category of representations of $A$ graded with respect to $A_{0}$ are equivalent naturally.

For any $\mu$-representation $r$ of $A_{0}$ in a purely even Hilbert space $\mathcal{H}(r)$, let

$$
R_{r}:=\operatorname{Ind}_{A_{0}}^{A} r
$$

be the representation of $A$ induced by $r$. We recall that $R_{r}$ acts in the Hilbert space $\mathcal{H}\left(R_{r}\right)$ of all (equivalence classes of Borel) functions $f(A \rightarrow \mathcal{H}(r))$ such that for each $\alpha \in A_{0}$,

$$
f(\alpha a)=\mu(\alpha, a) r(\alpha) f(a)
$$

for almost all $a \in A$; and

$$
\left(R_{r}(a) f\right)(y)=\mu(y, a) f(y a) \quad(a, y \in A) .
$$

The space $\mathcal{H}\left(R_{r}\right)$ is naturally graded by defining

$$
\mathcal{H}\left(R_{r}\right)_{j}=\left\{f \in \mathcal{H}\left(R_{r}\right) \mid \operatorname{supp}(f) \subset A_{j}\right\} \quad(j=0,1) .
$$

It is then obvious that $R_{r}$ is a graded $\mu$-representation. We write $\widehat{R_{r}}$ for $R_{r}$ treated as a graded representation. These remarks suggest the following lemma.

Lemma 15 For any unitary $\mu$-representation $r$ of $A_{0}$ let $R_{r}=\operatorname{Ind}(r)$ and let $\widehat{R_{r}}$ be the graded $\mu$-representation defined by $R_{r}$. Then the assignment $r \mapsto \widehat{R_{r}}$ is an equivalence from the category of unitary $\mu$-representations of $A_{0}$ to the category of unitary graded $\mu$-representations of $A$.

Proof. Let us first assume that $\mu=1$. Then we are dealing with UR's and the above remarks imply the Lemma in view of the classical imprimitivity theorem.

When $\mu$ is not 1 we go to the central extension $A^{\sim}$ of $A$ by $\mathbb{Z}_{2}$ defined by $\mu$. Recall that $A^{\sim}=A \times_{\mu} \mathbb{Z}_{2}$ with multiplication defined by

$$
(a, \xi)\left(a^{\prime}, \xi^{\prime}\right)=\left(a a^{\prime}, \xi \xi^{\prime} \mu\left(a, a^{\prime}\right)\right) \quad\left(a, a^{\prime} \in A, \xi, \xi^{\prime} \in \mathbb{Z}_{2}\right)
$$


(We must give to $A^{\sim}$ the Weil topology). Then $A_{0}^{\sim}=A_{0} \times_{\mu} \mathbb{Z}_{2}$ and $A^{\sim} / A_{0}^{\sim}=$ $A / A_{0}$. The $\mu$-representations $R$ of $A$ are in natural bijection with UR's $R^{\sim}$ of $A^{\sim}$ such that $R^{\sim}$ is nontrivial on $\mathbb{Z}_{2}$ by the correspondence

$$
R^{\sim}(a, \xi)=\xi R(a), \quad R(a)=R^{\sim}(a, 1) .
$$

The assignment $R \mapsto R^{\sim}$ is an equivalence of categories. Analogous considerations hold for $\mu$-representations $r$ of $A_{0}$ and UR's $r^{\sim}$ of $A_{0}^{\sim}$ which are nontrivial on $\mathbb{Z}_{2}$. The Lemma would now follow if we establish two things:

(a) For any unitary $\mu$-representation $r$ of $A_{0}$, and $R_{r}=\operatorname{Ind}(r)$, we have

$$
R_{r}^{\sim} \simeq \operatorname{Ind}\left(r^{\sim}\right)
$$

and (b) If $\rho$ is a UR of $A_{0}^{\sim}$ and $\operatorname{Ind}(\rho)=R^{\sim}$ for some $\mu$-representation $R$ of $A$, then $\rho=r^{\sim}$ for some $\mu$-representation $r$ of $A_{0}$. To prove (a) we set up the map $f \longmapsto f^{\sim}$ from $\mathcal{H}\left(R_{r}^{\sim}\right)$ to $\mathcal{H}\left(\operatorname{Ind}\left(r^{\sim}\right)\right)$ by

$$
f^{\sim}(a, \xi)=f(a) \xi
$$

It is an easy calculation that this is an isomorphism of $R_{r}^{\sim}$ with $\mathcal{H}\left(\operatorname{Ind}\left(r^{\sim}\right)\right)$ that intertwines the two projection valued measures on $A / A_{0}$ and $A^{\sim} / A_{0}^{\sim} \approx$ $A / A_{0}$. To prove (b) we have only to check that $\rho(1, \xi)=\xi$; this however is a straightforward calculation.

Remark 3 Given a graded $\mu$-representation $R$ of $A$, let $r$ be the $\mu$-representation of $A_{0}$ defined by

$$
r(\alpha)=\left.R(\alpha)\right|_{\mathcal{H}(R)_{0}} \quad\left(\alpha \in A_{0}\right) .
$$

It is then easy to show that $R \simeq R_{r}$. In fact it is enough to verify this (as before) when $\mu=1$. In this simple situation this is well known.

We now resume our discussion and treat the odd dimensional case.

Case II.b. $\operatorname{dim}\left(W^{\sim}\right)$ is odd.

We shall exhibit a projective even UR $\kappa$ of $H$ satisfying $(*)$. We refer back to the construction of $\tau^{\sim}$ in Lemma 11. Then

$$
S=\left(\begin{array}{cc}
0 & 1 \\
-1 & 0
\end{array}\right)
$$

is an odd unitary operator such that $S^{2}=-1$ and $\tau^{\sim}(x) S=(-1)^{p(x)} S \tau^{\sim}(x)$ for all $x \in \mathcal{C}^{\sim}$. Let $\gamma\left(\mathrm{O}\left(W^{\sim}\right) \rightarrow G\right)$ be a Borel right inverse of $\alpha$. Then, it is easily checked that

$$
\kappa_{H}(h)= \begin{cases}\left(\tau^{\sim} \circ \gamma \circ j\right)(h) & \text { if } h \in H_{0} \\ \left(\tau^{\sim} \circ \gamma \circ j\right)(h) S & \text { if } h \in H \backslash H_{0}\end{cases}
$$


has the required properties.

We now return to our original setting. First the graded representations of $H$ are obtained by taking $H=A, H_{0}=A_{0}$ in the foregoing discussion. Let $\mathfrak{g}_{1 \lambda}=\mathfrak{g}_{1} / \operatorname{rad} \Phi_{\lambda}$. We write $\mathcal{C}_{\lambda}$ for the algebra generated by $\mathfrak{g}_{1}$ with the relations $X^{2}=Q_{\lambda}(X) 1$ for all $X \in \mathfrak{g}_{1}$. We have a map

$$
j_{\lambda}: L_{0}^{\lambda} \longrightarrow \mathrm{O}\left(\mathfrak{g}_{1 \lambda}\right)
$$

We take in the preceding theory

$$
q=Q_{\lambda}, \quad \varphi=\Phi_{\lambda}, \quad H=L_{0}^{\lambda}, \quad W^{\sim}=\mathfrak{g}_{1 \lambda}, \quad j=j_{\lambda}, \quad \mathcal{C}^{\sim}=\mathcal{C}_{\lambda}^{\sim}
$$

Furthermore let

$$
\kappa_{\lambda}=\kappa, \quad \mu_{\lambda}=\mu, \quad \tau_{\lambda}^{\sim}=\tau^{\sim}, \quad \tau_{\lambda}=\text { lift of } \tau^{\sim} \text { to } \mathcal{C}_{\lambda}
$$

Then $\mu_{\lambda}$ is a normalized multiplier for $L_{0}^{\lambda}$ which we can choose to be \pm valued, $\kappa_{\lambda}$ is a $\mu_{\lambda}$-representation (unitary) of $L_{0}^{\lambda}$ in the space of $\tau_{\lambda}$, and

$$
\kappa_{\lambda}(t) \tau_{\lambda}(X) \kappa_{\lambda}(t)^{-1}=\tau_{\lambda}(t X) \quad\left(t \in L_{0}^{\lambda}\right)
$$

Moreover, $\kappa_{\lambda}$ is graded if and only if $j_{\lambda}\left(L_{0}^{\lambda}\right) \not \subset \mathrm{SO}\left(\mathfrak{g}_{1 \lambda}\right)$ and $\operatorname{dim}\left(\mathfrak{g}_{1 \lambda}\right)$ is even, otherwise $\kappa_{\lambda}$ is even. Finally, let

$$
L_{00}^{\lambda}= \begin{cases}j_{\lambda}^{-1}\left(\mathrm{SO}\left(\mathfrak{g}_{1 \lambda}\right)\right) & \text { if } j_{\lambda}\left(L_{0}^{\lambda}\right) \not \subset \mathrm{SO}\left(\mathfrak{g}_{1 \lambda}\right) \text { and } \operatorname{dim}\left(\mathfrak{g}_{1 \lambda}\right) \text { is even } \\ L_{0}^{\lambda} & \text { otherwise. }\end{cases}
$$

For any unitary $\mu_{\lambda}$-representation $r$ of $L_{00}^{\lambda}$ in an even Hilbert space $\mathcal{K}_{\lambda}$, let $\widehat{R_{r}}$ be the unitary $\mu_{\lambda^{-}}$representation of $L_{0}^{\lambda}$ induced by $r$, which is graded if $j_{\lambda}\left(L_{0}^{\lambda}\right) \not \subset \mathrm{SO}\left(\mathfrak{g}_{1 \lambda}\right)$ and $\operatorname{dim}\left(\mathfrak{g}_{1 \lambda}\right)$ is even, and is just $r$ in all other cases.

Theorem 4 Let $\lambda$ be such that $\Phi_{\lambda} \geq 0$. Then $\lambda$ is admissible, i.e., $\lambda \in T_{0}^{+}$. For a fixed such $\lambda$ let $\tau_{\lambda}$ be an irreducible SA representation of $\mathcal{C}_{\lambda}$ in a SHS $\mathcal{L}_{\lambda}$ and $\kappa_{\lambda}$ the unitary $\mu_{\lambda}$-representation of $L_{0}^{\lambda}$ in $\mathcal{L}_{\lambda}$ associated to $\tau_{\lambda}$ as above. For any unitary $\mu_{\lambda}$-representation $r$ of $L_{00}^{\lambda}$ in an even Hilbert space $\mathcal{K}_{\lambda}$, let $\widehat{R_{r}}$ be the unitary $\mu_{\lambda}$-representation of $L_{0}^{\lambda}$ defined as above, and let

$$
\theta_{r \lambda}=\left(\sigma_{r \lambda}, \rho_{\lambda}^{\sigma}\right)
$$

be the UR of the little SLG $S^{\lambda}$, where, for $X \in \mathfrak{g}_{1}, h \in L_{0}^{\lambda}, t \in T_{0}$,

$$
\sigma_{r \lambda}(t h)=e^{i \lambda(t)} \sigma_{r \lambda}^{\prime}(h)
$$


and

$$
\sigma_{r \lambda}^{\prime}(h)=\widehat{R_{r}}(h) \otimes \kappa_{\lambda}(h), \quad \rho_{\lambda}^{\sigma}(X)=1 \otimes \tau_{\lambda}(X) \quad\left(X \in \mathfrak{g}_{1}\right) .
$$

Then $\theta_{r \lambda}$ is an admissible UR of $S^{\lambda}$. The assignment $r \longmapsto \theta_{r \lambda}$ is functorial, commutes with direct sums, and is an equivalence of categories from the category of unitary $\mu_{\lambda}$-representations of $L_{00}^{\lambda}$ to the category of admissible UR's of the little super group $S^{\lambda}$. If $L_{0}^{\lambda}$ is connected and satisfies either of the conditions of Lemma 13, then $r \longmapsto \theta_{r \lambda}$ is an equivalence from the category of even UR's of $L_{0}^{\lambda}$ into the category of admissible UR's of $S^{\lambda}$.

Proof. Once $\kappa_{\lambda}$ is fixed, the assignment

$$
r \longmapsto \theta_{r \lambda}
$$

is clearly functorial (although it depends on $\kappa_{\lambda}$ ). If $\operatorname{dim}\left(\mathfrak{g}_{1 \lambda}\right)$ is even, a morphism $M: r_{1} \longrightarrow r_{2}$ obviously gives rise to the morphism $\widehat{M}: R_{r_{1}} \longrightarrow$ $R_{r_{2}}$ and hence to the morphism $\widehat{M} \otimes 1$ from $\theta_{r_{1} \lambda}$ to $\theta_{r_{2} \lambda}$. Conversely, if $T$ is a bounded even operator commuting with $1 \otimes \tau_{\lambda}$, it is immediate (since the $\tau_{\lambda}(X)$ generate the full super algebra of endomorphisms of $\mathcal{L}_{\lambda}$ ) that $T$ must be of the form $M^{\prime} \otimes 1$ where $M^{\prime}: \mathcal{H}\left(\widehat{R}_{r_{1}}\right) \rightarrow \mathcal{H}\left(\widehat{R}_{r_{2}}\right)$ is a bounded even operator. If now $T$ intertwines $\widehat{R}_{r_{1}} \otimes \kappa_{\lambda}$ and $\widehat{R}_{r_{2}} \otimes \kappa_{\lambda}$, then $M^{\prime}$ must belong to $\operatorname{Hom}\left(\widehat{R}_{r_{1}}, \widehat{R}_{r_{2}}\right) \approx \operatorname{Hom}\left(r_{1}, r_{2}\right)$. Thus $r \longmapsto \theta_{r \lambda}$ is a fully faithful functor. If $\operatorname{dim}\left(\mathfrak{g}_{1 \lambda}\right)$ is odd, we can choose $\mathcal{K}_{\lambda}$ to be purely even (see Lemma 111). If $T$ is a bounded even operator commuting with $1 \otimes \tau_{\lambda}$, we use the fact that it commutes with

$$
1 \otimes\left(\begin{array}{cc}
\tau^{+}(a) & 0 \\
0 & \tau^{+}(a)
\end{array}\right) \text { and } 1 \otimes\left(\begin{array}{ll}
0 & 1 \\
1 & 0
\end{array}\right)
$$

(in the notation of Lemma 111) to conclude, via an argument similar to the one used in the even dimensional case, that $T$ is of the form $M \otimes 1$. Arguing as before we conclude that $M \in \operatorname{Hom}\left(r_{1}, r_{2}\right)$. Thus $r \mapsto \theta_{r \lambda}$ is a fully faithful functor in this case also. It remains to show that every admissible UR of $S^{\lambda}$ is of the form $\theta_{r \lambda}$.

Let $\theta$ be an admissible UR of $S^{\lambda}$ in $\mathcal{H}$. Then $\theta=(\xi, \tau)$ where $\xi$ is an even UR of $T_{0} L_{0}^{\lambda}$ which restricts to $e^{i \lambda} I$ on $T_{0}, \tau$ is a SA representation of $\mathcal{C}_{\lambda}$ related to $\xi$ as usual. We may then assume by Lemma 11 that $\mathcal{H}=\mathcal{K} \otimes \mathcal{L}_{\lambda}$ and $\tau=1 \otimes \tau_{\lambda}$. If $\operatorname{dim}\left(\mathfrak{g}_{1 \lambda}\right)$ is odd, we choose $\mathcal{K}$ purely even. Then $1 \otimes \tau_{\lambda}(h X)=$ $\xi(h)\left[1 \otimes \tau_{\lambda}(X)\right] \xi(h)^{-1}$. But the same relation is true if we replace $\xi$ by $1 \otimes \kappa_{\lambda}$. So if $\xi_{1}=\left[1 \otimes \kappa_{\lambda}\right]^{-1} \xi$, then $\xi_{1}(h)$ is even or odd according to the grading of 
$\kappa_{\lambda}(h)$, and commutes with $1 \otimes \tau_{\lambda}$. Hence $\xi_{1}$ is of the form $R^{\prime} \otimes 1$ for a Borel map $R^{\prime}$ from $L_{0}^{\lambda}$ into the unitary group of $\mathcal{K}$. Thus

$$
\xi(h)=\left[1 \otimes \kappa_{\lambda}(h)\right]\left[R^{\prime}(h) \otimes 1\right]
$$

The two factors on the right side of this equation commute; the left side is an even UR and the first factor on the right is a unitary $\mu_{\lambda}$-representation of $L_{0}^{\lambda}$, which is graded or even according to $\operatorname{dim}\left(\mathfrak{g}_{1 \lambda}\right)$ even or $\operatorname{dim}\left(\mathfrak{g}_{1 \lambda}\right)$ odd. So $R^{\prime}$ is a $\mu_{\lambda}^{-1}=\mu_{\lambda}$-representation of $L_{0}^{\lambda}$ in $\mathcal{K}$, which is graded or even according to $\operatorname{dim}\left(\mathfrak{g}_{1 \lambda}\right)$ even or $\operatorname{dim}\left(\mathfrak{g}_{1 \lambda}\right)$ odd. This finishes the proof.

Remark 4 If $\lambda=0$, then $\Phi_{\lambda}=0, \mathcal{L}_{\lambda}=0$, and $\theta_{r 0}=(r, 0)$.

Remark 5 For the super Poincaré groups we shall see in the next subsection that the situation is much simpler and $L_{0}^{\lambda}$ is always connected and satisfies the conditions of Lemma 13.

Combining Theorems 3 and 4 we obtain the following theorem. Let $\Theta_{r \lambda}$ be the UR of $\left(G_{0}, \mathfrak{g}\right)$ induced by $\theta_{r \lambda}$ as described in Theorem 4 .

Theorem 5 Let $\lambda$ be such that $\Phi_{\lambda} \geq 0$. The assignment that takes $r$ to the UR $\Theta_{r \lambda}$ is an equivalence of categories from the category of unitary $\mu_{\lambda^{-}}$ representations of $L_{00}^{\lambda}$ to the category of UR's of $\left(G_{0}, \mathfrak{g}\right)$ whose spectra are contained in the orbit of $\lambda$. In particular, for $r$ irreducible, $\Theta_{r \lambda}$ is irreducible, and every irreducible UR of $\left(G_{0}, \mathfrak{g}\right)$ is obtained in this way. If the conditions of Lemma 13 are satisfied, then the $r$ 's come from the category of UR's of $L_{0}^{\lambda}$.

In the case of super Poincaré groups (see Remark 5 above), $\Theta_{r \lambda}$ induced by $\theta_{r \lambda}$ represents a superparticle. In general the UR $\pi_{r \lambda}$ of $T_{0} L_{0}$ contained in $\Theta_{r \lambda}$ will not be an irreducible UR of $G_{0}$. Its decomposition into irreducibles gives the multiplet that the UR of $S$ determines. This is of course the set of irreducible UR's $U_{r \lambda j}$ of $G_{0}$ induced by the $r_{\lambda j}$ where the $r_{\lambda j}$ are the irreducible UR's of $L_{0}^{\lambda}$ contained in $r \otimes \kappa_{\lambda}$ :

$$
r \otimes \kappa_{\lambda}=\bigoplus r_{\lambda j}, \quad \pi_{r \lambda}=\bigoplus U_{r \lambda j}
$$

The set $\left(r_{\lambda j}\right)$ thus defines the multiplet. For $r$ trivial the corresponding multiplet is called fundamental. 


\subsection{The case of the super Poincaré groups}

We shall now specialize the entire theory to the case when $\left(G_{0}, \mathfrak{g}\right)$ is a super Poincaré group (SPG). This means that the following conditions are satisfied.

(a) $T_{0}=\mathbb{R}^{1, D-1}$ is the $D$-dimensional Minkowski space of signature $(1, D-$ 1) with $D \geq 4$; the Minkowski bilinear form is $\left\langle x, x^{\prime}\right\rangle=x_{0} x_{0}^{\prime}-\sum_{j} x_{j} x_{j}^{\prime}$.

(b) $L_{0}=\operatorname{Spin}(1, D-1)$.

(c) $\mathfrak{g}_{1}$ is a real spinorial module for $L_{0}$, i.e., is a direct sum of spin representations over $\mathbb{C}$.

(d) For any $0 \neq X \in \mathfrak{g}_{1}$, and any $x \in T_{0}$ lying in the interior $\Gamma^{+}$of the forward light cone $\Gamma$, we have

$$
\langle[X, X], x\rangle>0
$$

If in (c) $\mathfrak{g}_{1}$ is the sum of $N$ real irreducible spin modules of $L_{0}$, we say we are in the context of $N$-extended supersymmetry. Sometimes $N$ refers to the number of irreducible components over $\mathbb{C}$. In (d)

$$
\Gamma=\left\{x \mid\langle x, x\rangle \geq 0, x_{0} \geq 0\right\}, \quad \Gamma^{+}=\left\{x \mid\langle x, x\rangle>0, x_{0}>0\right\} .
$$

In the case when $D=4$ and $\mathfrak{g}_{1}$ is the Majorana spinor, the condition (d) is automatic (one may have to change the sign of the odd commutators to achieve this); in the general case, as we shall see below, it ensures that only positive energy representations are allowed.

We identify $T_{0}^{*}$ with $\mathbb{R}^{1, D-1}$ by the pairing $\langle x, p\rangle=x_{0} p_{0}-\sum_{j} x_{j} p_{j}$. The dual action of $L_{0}$ is then the original action. The orbit structure of $T_{0}^{*}$ is classical.

Lemma 16 ( $i$ ) Let $V$ be a finite dimensional real vector space with a nondegenerate quadratic form and let $V_{1}$ be a subspace of $V$ on which the quadratic form remains nondegenerate. Then the spin representations of $\operatorname{Spin}(V)$ restrict on $\operatorname{Spin}\left(V_{1}\right)$ to direct sums of spin representations of $\operatorname{Spin}\left(V_{1}\right)$. (ii) Suppose $V=\mathbb{R}^{1, D-1}$. Let $p \in V$ be such that $\langle p, p\rangle= \pm m^{2} \neq 0$ and $V_{1}=p^{\perp}$. Then $V_{1}$ is a quadratic subspace, the stabilizer $L_{0}^{p}$ of $p$ in $\operatorname{Spin}(V)$ is precisely $\operatorname{Spin}\left(V_{1}\right)$, and it is $\simeq \operatorname{Spin}(D-1)$ for $\langle p, p\rangle=m^{2}$ and $\simeq \operatorname{Spin}(1, D-2)$ for $\langle p, p\rangle=-m^{2} \neq 0$. 
Proof. (i) Let $\mathcal{C}, \mathcal{C}_{1}$ be the Clifford algebras of $V$ and $V_{1}$. Then $\mathcal{C}_{1}^{+} \subset \mathcal{C}^{+}$ and hence, as the spin groups are imbedded in the even parts of the Clifford algebras, we have $\operatorname{Spin}\left(V_{1}\right) \subset \operatorname{Spin}(V)$. Now the spin modules are precisely the modules for the even parts of the corresponding Clifford algebras and so, as these algebras are semisimple, the decomposition of the spin module of $\operatorname{Spin}(V)$, viewed as an irreducible module for $\mathcal{C}^{+}$, into irreducible modules for $\mathcal{C}_{1}^{+}$under restriction to $\mathcal{C}_{1}^{+}$, gives the decomposition of the restriction of the original spin module to $\operatorname{Spin}\left(V_{1}\right)$. See [Del99, , Var04.

(ii) Choose an orthogonal basis $\left(e_{\alpha}\right)_{0 \leq \alpha \leq D-1}$ such that $\left\langle e_{0}, e_{0}\right\rangle=-\left\langle e_{j}, e_{j}\right\rangle=$ 1 for $1 \leq j \leq D-1$. It is easy to see that we can move $p$ to either $(m, 0, \ldots, 0)$ or $(0, m, 0, \ldots, 0)$ by $L_{0}$ and so we may assume that $p$ is in one of these two positions. For $u \in C(V)^{+}$it is then a straightforward matter to verify that $u p=p u$ if and only if $u \in C\left(V_{1}\right)^{+}$. From the characterization of the spin group (Del99, , Var04]) it is now clear that $L_{0}^{p}=\operatorname{Spin}\left(V_{1}\right)$.

Lemma 17 Let $M$ be a connected real semisimple Lie group whose universal cover does not have a compact factor, i.e., the Lie algebra of $M$ does not have a factor Lie algebra whose group is compact. Then $M$ has no nontrivial morphisms into any compact Lie group, and hence no nontrivial finite dimensional UR's.

Proof. We may assume that $M$ is simply connected. If such a morphism exists we have a nontrivial morphism $\mathfrak{m} \longrightarrow \mathfrak{k}$ where $\mathfrak{m}$ is the Lie algebra of $M$ and $\mathfrak{k}$ is the Lie algebra of a compact Lie group. Let $\mathfrak{a}$ be the kernel of this Lie algebra morphism. Then $\mathfrak{a}$ is an ideal of $\mathfrak{m}$ different from $\mathfrak{m}$, and so we can write $\mathfrak{m}$ as $\mathfrak{a} \times \mathfrak{k}^{\prime}$ where $\mathfrak{k}^{\prime}$ is also an ideal and is non zero; moreover, the map from $\mathfrak{k}^{\prime}$ to $\mathfrak{k}$ is injective. $\mathfrak{k}^{\prime}$ is semisimple and admits an invariant negative definite form (the restriction from the Cartan- Killing form of $\mathfrak{k}$ ), and so its associated simply connected group $K^{\prime}$ is compact. If $A$ is the simply connected group for $\mathfrak{a}$, we have $M=A \times K^{\prime}$, showing that $M$ admits a compact factor, contrary to hypothesis.

Corollary 1 If $V$ is a quadratic vector space of signature $(p, q), p, q>0, p+$ $q \geq 3$, then $\operatorname{Spin}(V)$ does not have any nontrivial map into a compact Lie group.

Proof. The Lie algebra is semisimple and the simple factors are not compact.

Lemma 18 We have $\Gamma=\left\{p \mid \Phi_{p} \geq 0\right\}$, i.e., for any $p \in \mathbb{R}^{1, D-1}$,

$$
\Phi_{p} \geq 0 \Longleftrightarrow p_{0} \geq 0,\langle p, p\rangle \geq 0 .
$$


Moreover,

$$
p_{0}>0,\langle p, p\rangle>0 \Longrightarrow \Phi_{p}>0 .
$$

Proof. For $0 \neq X \in \mathfrak{g}_{1}$ we have $\langle[X, X], x\rangle>0$ for all $x \in \Gamma^{+}$and hence the inequality is true with $\geq 0$ replacing $>0$ for $x \in \Gamma$. Hence $2 \Phi_{p}(X, X)=$ $\langle[X, X], p\rangle \geq 0$ if $p \in \Gamma$. So

$$
\Gamma \subset\left\{p \mid \Phi_{p} \geq 0\right\} .
$$

We shall show next that

$$
\left\{p \mid \Phi_{p} \geq 0\right\} \subset\{p \mid\langle p, p\rangle \geq 0\} .
$$

Suppose on the contrary that $\Phi_{p} \geq 0$ but $\langle p, p\rangle<0$. Since $\Phi_{p}$ is invariant under $L_{0}^{p}$ which is connected, we have a map $L_{0}^{p} \longrightarrow \mathrm{SO}\left(\mathfrak{g}_{1 p}\right)$. Then $L_{0}^{p}=$ $\operatorname{Spin}\left(V_{1}\right)=\operatorname{Spin}(1, D-2)$ by Lemma 16, and Corollary 1 shows that $L_{0}^{p}$ has no nontrivial morphisms into any compact Lie group. Hence $L_{0}^{p}$ acts trivially on $\mathfrak{g}_{1 p}$. Since $L_{0}^{p}$ is a semisimple group, $\mathfrak{g}_{1 p}$ can be lifted to an $L_{0}^{p}$-invariant subspace of $\mathfrak{g}_{1}$. Hence, if $\mathfrak{g}_{1 p} \neq 0$, the action of $L_{0}^{p}$ on $\mathfrak{g}_{1}$ must have non zero trivial submodules. However, by Lemma 16, the spin modules of $\operatorname{Spin}(V)$ restrict on $\operatorname{Spin}\left(V_{1}\right)$ to direct sums of spin modules of the smaller group and there is no trivial module in this decomposition. Hence $\mathfrak{g}_{1 p}=0$, i.e., $\Phi_{p}=0$. Hence $p$ vanishes on $\left[\mathfrak{g}_{1}, \mathfrak{g}_{1}\right]$. Now $\left[\mathfrak{g}_{1}, \mathfrak{g}_{1}\right]$ is stable under $L_{0}$ and non zero, and so must be the whole of $\mathfrak{t}_{0}$. So $p=0$, a contradiction.

To finish the proof we should prove that if $\Phi_{p} \geq 0$ then $p_{0} \geq 0$. Otherwise $p_{0}<0$ and so $-p \in \Gamma$ and so from what we have already proved, we have $\Phi_{-p}=-\Phi_{p} \geq 0$. Hence $\Phi_{p}=0$. But then as before $p=0$, a contradiction.

Finally, if $p_{0}>0$ and $\langle p, p\rangle>0$, then $\Phi_{p}>0$ by definition of the SPG structure. This completes the proof.

Theorem 6 Let $S=\left(G_{0}, \mathfrak{g}\right)$ be a SPG. Then all stabilizers are connected and

$$
T_{0}^{+}=\left\{p \mid \Phi_{p} \geq 0\right\}=\Gamma .
$$

Moreover, $\kappa_{p}$ is an even UR of $L_{0}^{p}$, and the irreducible UR's of $S$ whose spectra are in the orbit of $p$ are in natural bijection with the irreducible UR's of $L_{0}^{p}$. The corresponding multiplet is then the set of irreducible UR's parametrized by the irreducibles of $L_{0}^{p}$ occurring in the decomposition of $\alpha \otimes \kappa_{p}$ as a UR of $L_{0}^{p}$.

Proof. In view of Theorem 4 and Lemma 18 we have $T_{0}^{+}=\Gamma$. For $p \in \Gamma$, the stabilizers are all known classically. If $\langle p, p\rangle>0, L_{0}^{p}=\operatorname{Spin}(D-1)$; if 
$\langle p, p\rangle=0$ but $p_{0}>0$, then $L_{0}^{p}=\mathbb{R}^{D-2} \times^{\prime} \operatorname{Spin}(D-2)$; and for $p=0, L_{0}^{p}=L_{0}$. So, except when $D=4$ and $p$ is non zero and is in the zero mass orbit, the stabilizer is connected and simply connected, thus $\kappa_{p}$ is an even $U R$ of $L_{0}^{p}$ by Lemma [13. But in the exceptional case, $L_{0}^{p}=\mathbb{R}^{2} \times^{\prime} S^{1}$ where $S^{1}$ is the circle, and Lemma 13 is again applicable. This finishes the proof.

\subsection{Determination of $\kappa_{p}$ and the structure of the mul- tiplets. Examples}

We have seen that the multiplet defined by the super particle $\Theta_{\alpha p}$ is parametrized by the set of irreducible UR's of $L_{0}^{p}$ that occur in the decomposition of $\alpha \otimes \kappa_{p}$. Clearly it is desirable to determine $\kappa_{p}$ as explicitly as possible. We shall do this in what follows.

To determine $\kappa_{p}$ the following lemma is useful. $(W, q)$ is a positive definite quadratic vector space and $\varphi$ is the bilinear form of $q . C(W)$ is the Clifford algebra of $W$ and $H$ is a connected Lie group with a morphism $H \longrightarrow \mathrm{SO}(W)$. $\tau$ is an irreducible SA representation of $C(W)$ and $\kappa$ is a UR such that $\kappa(t) \tau(u) \kappa(t)^{-1}=\tau(t u)$ for all $u \in W, t \in H$. We write $\approx$ for equivalence after multiplying by a suitable character.

Lemma 19 Suppose that $\operatorname{dim}(W)=2 m$ is even and $W_{\mathbb{C}}:=\mathbb{C} \otimes_{\mathbb{R}} W$ has an isotropic subspace $E$ of dimension $m$ stable under $H$. Let $\eta$ be the action of $H$ on $\Lambda(E)$ extending its action on $E$. Then

$$
\kappa \approx \Lambda(E) \approx \Lambda\left(E^{*}\right) \quad\left(E^{*} \text { is the complex conjugate of } E\right) .
$$

Proof. Clearly $E^{*}$ is also isotropic and $H$-stable. $E \cap E^{*}=0$ as otherwise $E \cap E^{*} \cap W$ will be a non zero isotropic subspace of $W$. So $W_{\mathbb{C}}=E \oplus E^{*}$. We write $\tau^{\prime}$ for the representation of $C(W)$ in $\Lambda(E)$ where

$$
\tau^{\prime}(u)(x)=u \wedge x, \quad \tau^{\prime}(v)(x)=\partial(v)(x) \quad\left(u \in E, v \in E^{*}, x \in \Lambda(E)\right) .
$$

Here $\partial(v)$ is the odd derivation taking $x \in E$ to $2 \varphi(x, v)$. It is then routine to show that

$$
\eta(t) \tau^{\prime}(u) \eta(t)^{-1}=\tau^{\prime}(t u) \quad\left(u \in E \cup E^{*}\right) .
$$

Now $\tau^{\prime}$ is equivalent to $\tau$ and so we can transfer $\eta$ to an action, written again as $\eta$, of $H$ in the space of $\tau$ satisfying the above relation with respect to $\tau$. It is not necessary that $\eta$ be unitary. But we can normalize it to be a UR, namely $\kappa(t)=|\operatorname{det}(\eta(t))|^{-1 / \operatorname{dim}(\tau)} \eta(t)$. 
Remark 6 It is easy to give an independent argument that $\Lambda(E) \approx \Lambda\left(E^{*}\right)$. For the unitary group $\mathrm{U}(E)$ of $E$ let $\Lambda_{r}$ be the representation on $\Lambda^{r}(E)$, and let $\Lambda$ be their direct sum; then a simple calculation of the characters on the diagonal group shows that $\Lambda_{r}{ }^{*} \simeq \operatorname{det}^{-1} \otimes \Lambda_{n-r}$. Hence $\Lambda^{*} \simeq \operatorname{det}^{-1} \otimes \Lambda$, showing that $\Lambda^{*} \approx \Lambda$. It is then immediate that this result remains true for any group which acts unitarily on $E$.

Corollary 2 The conditions of the above lemma are met if $W=A \oplus B$ where $A, B$ are orthogonal submodules for $H$ which are equivalent. Moreover

$$
\kappa \approx \Lambda(E) \simeq \Lambda\left(E^{*}\right) \simeq \Lambda(A) \simeq \Lambda(B) .
$$

Proof. Take ON bases $\left(a_{j}\right),\left(b_{j}\right)$ for $A$ and $B$ respectively so that the map $a_{j} \mapsto b_{j}$ is an isomorphism of $H$-modules. If $E$ is the span of the $e_{j}=$ $a_{j}+i b_{j}$, it is easy to check that $E$ is isotropic, and is a module for $H$ which is equivalent to $A$ and $B$.

We now assume that for some $r \geq 3$ we have a map

$$
H \longrightarrow \operatorname{Spin}(r) \longrightarrow \operatorname{Spin}(W)
$$

where the first map is surjective, and $H$ acts on $W$ through $\operatorname{Spin}(W)$. Further let the representation of $\operatorname{Spin}(r)$ on $W$ be spinorial. We write $\sigma_{r}$ for the (complex) spin representation of $\operatorname{Spin}(r)$ if $r$ is odd and $\sigma_{r}^{ \pm}$for the (complex) spin representations of $\operatorname{Spin}(r)$ if $r$ is even. Likewise we write $s_{r}, s_{r}^{ \pm}$for the real irreducible spin modules. Note that $\operatorname{dim}(W)$ must be even.

Lemma 20 Let the representation of $\operatorname{Spin}(r)$ on $W$ be spinorial. Let $n$ be the number of real irreducible constituents of $W$ as a module for $\operatorname{Spin}(r)$, and, when $r$ is even, let $n^{ \pm}$be the number of irreducible constituents of real or quaternionic type. We then have the following determination of $\kappa$.

$$
\begin{array}{ll}
r \text { mod } 8 & \kappa \\
0\left(n^{ \pm} \text {even }\right) & \Lambda\left(\left(\left(n^{+} / 2\right) \sigma_{r}^{+} \oplus\left(n^{-} / 2\right) \sigma_{r}^{-}\right)\right) \\
1,7(n \text { even }) & \Lambda\left((n / 2) \sigma_{r}\right) \\
2,6 & \Lambda\left(n \sigma_{r}^{+}\right) \approx \Lambda\left(n \sigma_{r}^{-}\right) \\
3,5 & \Lambda\left(n \sigma_{r}\right) \\
4 & \Lambda\left(n^{+} \sigma_{r}^{+} \oplus n^{-} \sigma_{r}^{-}\right)
\end{array}
$$

Proof. This is a routine application of the the Lemma and Corollary above if we note the following facts.

$$
r \equiv 0 \text { : Here } \sigma_{r}^{ \pm}=s_{r}^{ \pm} \text {and } W=n^{+} s_{r}^{+}+n^{-} s_{r}^{-} .
$$


$r \equiv 1,7:$ Here $\sigma_{r}=s_{r}, W=n s_{r}$.

$r \equiv 2,6$ : Over $\mathbb{C}, s_{r}$ becomes $\sigma_{r}^{+} \oplus \sigma_{r}^{-}$while $\sigma_{r}^{ \pm}$do not admit a non zero invariant form. So $W_{\mathbb{C}}=E \oplus E^{*}$ where $E=n \sigma_{r}^{+}, E^{*}=n \sigma_{r}^{-}$, and $q$ is zero on $E$.

$r \equiv 3,5: s_{r}$ is quaternionic, $W=n s_{r}, W_{\mathbb{C}}=2 n \sigma_{r}$ and $\sigma_{r}$ does not admit an invariant symmetric form.

$r \equiv 4: s_{r}^{ \pm}$are quaternionic and $\sigma_{r}^{ \pm}$do not admit a non zero invariant symmetric form; $W_{\mathbb{C}}=E \oplus E^{*}$, where $E=n^{+} \sigma_{r}^{+}+n^{-} \sigma_{r}^{-}$and $q$ is zero on E.

In deriving these the reader should use the results in Del99 and Var04 on the reality of the complex spin modules and the theory of invariant forms for them.

\subsubsection{Super Poincaré group associated to $\mathbb{R}^{1,3}: N=1$ supersymme- try}

Here $T_{0}=\mathbb{R}^{1,3}, L_{0}=\mathrm{SL}(2, \mathbb{C})_{\mathbb{R}}$ where the suffix $\mathbb{R}$ means that the complex group is viewed as a real Lie group. Let $\mathfrak{s}=\mathbf{2} \oplus \overline{\mathbf{2}}, \mathbf{2}$ being the holomorphic representation of $L_{0}$ in $\mathbb{C}^{2}$ and $\overline{\mathbf{2}}$ its complex conjugate. Thus we identify $\mathfrak{s}$ with $\mathbb{C}^{2} \oplus \mathbb{C}^{2}$ and introduce the conjugation on $\mathfrak{s}$ given by $\overline{(u, v)}=(\bar{v}, \bar{u})$. The action $(u, v) \mapsto(g u, \bar{g} v)$ of $L_{0}(\bar{g}$ is the complex conjugate of $g$ ) commutes with the conjugation and so defines the real form $\mathfrak{s}_{\mathbb{R}}$ invariant under $L_{0}$ (Majorana spinor). We take $\mathfrak{t}_{0}$ to be the space of $2 \times 2$ Hermitian matrices and the action of $L_{0}$ on it as $g, A \mapsto g A \bar{g}^{\mathrm{T}}$. For $\left(u_{i}, \overline{u_{i}}\right) \in \mathfrak{s}_{\mathbb{R}}(i=1,2)$ we put

$$
\left[\left(u_{1}, \overline{u_{1}}\right),\left(u_{2}, \overline{u_{2}}\right)\right]=\frac{1}{2}\left(u_{1}{\overline{u_{2}}}^{\mathrm{T}}+u_{2}{\overline{u_{1}}}^{\mathrm{T}}\right) .
$$

Then $\mathfrak{g}=\mathfrak{g}_{0} \oplus \mathfrak{g}_{1}$ with $\mathfrak{g}_{0}=\mathfrak{t}_{0} \oplus \mathfrak{l}_{0}, \mathfrak{g}_{1}=\mathfrak{s}_{\mathbb{R}}$ is a super Lie algebra and $\left(T_{0} L_{0}, \mathfrak{g}\right)$ is the SLG with which we are concerned.

Here $\mathbb{R}^{1,3} \simeq \mathfrak{t}_{0}$ by the map $a \mapsto h_{a}=\left(\begin{array}{cc}a_{0}+a_{3} & a_{1}-i a_{2} \\ a_{1}+i a_{2} & a_{0}-a_{3}\end{array}\right) ; \mathfrak{t}_{0} \simeq \mathfrak{t}_{0}^{*}$ with $p \in \mathfrak{t}_{0}$ viewed as the linear form $a \mapsto\langle a, p\rangle=a_{0} p_{0}-a_{1} p_{1}-a_{2} p_{2}-a_{3} p_{3}$. Then

$$
Q_{p}((u, \bar{u}))=\frac{1}{4} \bar{u}^{\mathrm{T}} h_{\check{p}} u, \quad \check{p}=\left(p_{0},-p_{1},-p_{2},-p_{3}\right) .
$$

$\mathrm{I}: p_{0}>0, m^{2}=\langle p, p\rangle>0$. We take $p=m I$ so that $L_{0}^{p}=\mathrm{SU}(2)$. Take $E=\{(u, 0)\}, E^{*}=\{(0, \bar{u})\}$. Then we are in the set up of Lemma 19, Then

$$
\kappa_{p}=\Lambda(E) \simeq 2 D^{0} \oplus D^{1 / 2}, \quad D^{j} \otimes \Lambda(E)=\left\{\begin{array}{l}
2 D^{j} \oplus D^{j+1 / 2} \oplus D^{j-1 / 2} \quad(j \geq 1 / 2) \\
2 D^{0} \oplus D^{1 / 2} \quad(j=0) .
\end{array}\right.
$$


Thus the multiplet with mass $m$ has the same mass $m$ and spins

$$
\left\{\begin{array}{l}
\{j, j, j+1 / 2, j-1 / 2\}(j>0) \\
\{0,0,1 / 2\}(j=0)
\end{array}\right.
$$

II $: p_{0}>0,\langle p, p\rangle=0$. Here we take $p=(1,0,0,-1), h_{p}=\left(\begin{array}{ll}0 & 0 \\ 0 & 2\end{array}\right)$. Then $L_{0}^{p}=\left(\begin{array}{ll}a & 0 \\ c & \bar{a}\end{array}\right)$. The characters $\chi_{n / 2}: a \mapsto a^{n}(n \in \mathbb{Z})$ are viewed as characters of $L_{0}^{p}$. Here $Q_{p}((u, \bar{u}))=\frac{1}{4} \bar{u}^{\mathrm{T}} h_{\check{p}} u=\left|u_{1}\right|^{2}$. The radical of $\Phi_{p}$ is the span of $\left(e_{2}, e_{2}\right)$ and $\left(i e_{2},-i e_{2}\right), e_{1}, e_{2}$ being the standard basis of $\mathbb{C}^{2}$. We identify $\mathfrak{g}_{1 p}$ with the span of $\left(e_{1}, e_{1}\right)$ and $\left(i e_{1},-i e_{1}\right)$. We now apply Lemma 19 with $E=\mathbb{C}\left(e_{1}, 0\right)$ which carries the character defined by $\chi_{1 / 2}$; then

$$
\Lambda(E)=\chi_{0} \oplus \chi_{1 / 2}, \quad \chi_{n / 2} \otimes \Lambda(E)=\chi_{n / 2} \oplus \chi_{(n+1) / 2} .
$$

The multiplet is $\{n / 2,(n+1) / 2\}$. These results go back to [SS74].

\subsubsection{Extended supersymmetry}

Here the SLG has still the Poincaré group as its even part but $\mathfrak{g}_{1}$ is the sum of $N>1$ copies of $\mathfrak{s}_{\mathbb{R}}$. It is known ([De199, Var04])that one can identify $\mathfrak{g}_{1}$ with the direct sum $\mathfrak{s}_{\mathbb{R}}^{N}$ of $N$ copies of $\mathfrak{s}_{\mathbb{R}}$ in such a way that for the odd commutators we have

$$
\left[\left(s_{1}, s_{2}, \ldots, s_{N}\right),\left(s_{1}^{\prime}, s_{2}^{\prime}, \ldots, s_{N}^{\prime}\right)\right]=\sum_{1 \leq i \leq N}\left[s_{i}, s_{i}^{\prime}\right]^{1}
$$

so that

$$
Q_{\lambda}\left(\left(s_{1}, \ldots, s_{N}\right)\right)=\sum_{1 \leq i \leq N} Q_{\lambda}^{1}\left(\left(s_{i}, s_{i}\right)\right)
$$

Here the index 1 means the [, ] and $Q$ for the case $N=1$ discussed above. Let $E^{N}=N E^{1}$.

I $: p_{0}>0, m^{2}=\langle p, p\rangle>0$. Then we apply Lemma 19 with $E=E^{N}$ so that $\kappa_{p}=\Lambda\left(N D^{1 / 2}\right)$. The decomposition of the exterior algebra of $N D^{1 / 2}$ is tedious but there is no difficulty in principle. We have

$$
\kappa_{p}=\sum_{0 \leq r \leq N} c_{N r} D^{r / 2} \quad c_{N r}>0, \quad c_{N N}=1 .
$$

Then $j+N / 2$ is the maximum value of $r$ for which $D^{r}$ occurs in $D^{j} \otimes$ $\Lambda\left(N D^{1 / 2}\right)$. The multiplet defined by the super particle of mass $m$ is thus

$$
\left\{\begin{array}{l}
\{j-N / 2, j-N / 2+1 / 2, \ldots, j+N / 2-1 / 2, j+N / 2\}(j \geq N / 2) \\
\{0,1 / 2, \ldots, j+N / 2-1 / 2, j+N / 2\}(0 \leq j<N / 2)
\end{array}\right.
$$


II $: p_{0}>0, m=0$. Here

$$
\kappa_{\lambda}=\Lambda\left(N \chi_{1 / 2}\right)=\sum_{0 \leq r \leq N}\left(\begin{array}{c}
N \\
r
\end{array}\right) \chi_{r / 2}
$$

The multiplet of the super particle has the helicity content

$$
\{r / 2,(r+1) / 2, \ldots,(r+N) / 2\}
$$

\subsubsection{Super particles of infinite spin}

The little groups for zero mass have irreducible UR's which are infinite dimensional. Since $L_{0}^{p}$ is also a semidirect product its irreducible UR's can be determined by the usual method. The orbits of $S^{1}$ in $\mathbb{C}$ (which is identified with its dual) are the circles $\{|a|=r\}$ for $r>0$ and the stabilizers of the points are all the same, the group $\{ \pm 1\}$. The irreducible UR's of infinite dimension can then be parametrized as $\left\{\alpha_{r, \pm}\right\}$. Now $\Lambda(E)=\chi_{0} \oplus \chi_{1 / 2}$ and an easy calculation gives

$$
\alpha_{r, \pm} \otimes \Lambda(E)=\alpha_{r,+} \oplus \alpha_{r,-} .
$$

The particles in the multiplet with mass 0 corresponding to spin $(r, \pm)$ consist of both types of infinite spin with the same $r$.

\subsubsection{Super Poincaré groups of Minkowski super spacetimes of arbitrary dimension}

Let $T_{0}=\mathbb{R}^{1, D-1}$. We first determine $\kappa_{p}$ in the massive case. Here $L_{0}^{p}=$ $\operatorname{Spin}(D-1)$ and the form $\Phi_{p}$ is strictly positive definite. So $\mathfrak{g}_{1 p}=\mathfrak{g}_{1}$ and $L_{0}^{p}$ acts on it by restriction, hence spinorially by Lemma 16. So Lemma 20 applies at once. It only remains to determine $n, n^{ \pm}$in terms of the corresponding $N, N^{ \pm}$for $\mathfrak{g}_{1}$ viewed as a module for $L_{0}$. Notation is as in Lemma 20, and res is restriction to $L_{0}^{p} ; r=D-1$. This is done by writing $\mathfrak{g}_{1}$ as a sum of the $s_{D}$ and determining the restrictions of the $s_{D}$ to $L_{0}^{p}$ by dimension counting. We again omit the details but refer the reader to [Del99, , Var04].

Proposition 8 When $p_{0}>0, m^{2}=\langle p, p\rangle>0, \kappa_{p}$, the fundamental multiplet 
of the super particle of mass $m$, is given according to the following table:

\begin{tabular}{|c|c|c|}
\hline$D \bmod 8$ & $\operatorname{res}\left(s_{D}\right)$ & $\kappa_{p}$ \\
\hline 0 & $2 s_{D-1}$ & $\Lambda\left(N \sigma_{D-1}\right)$ \\
\hline $1(N=2 k)$ & $s_{D-1}^{+}+s_{D-1}^{-}$ & $\Lambda\left(k \sigma_{D-1}^{+} \oplus k \sigma_{D-1}^{-}\right)$ \\
\hline $2(N=2 k)$ & $s_{D-1}$ & $\Lambda\left(k \sigma_{D-1}\right)$ \\
\hline 3 & $s_{D-1}$ & $\Lambda\left(N \sigma_{D-1}^{ \pm}\right)$ \\
\hline 4 & $s_{D-1}$ & $\Lambda\left(N \sigma_{D-1}\right)$ \\
\hline 5 & $s_{D-1}^{+}+s_{D-1}^{-}$ & $\Lambda\left(N \sigma_{D-1}^{+} \oplus N \sigma_{D-1}^{-}\right)$ \\
\hline 6 & $s_{D-1}$ & $\Lambda\left(N \sigma_{D-1}\right)$ \\
\hline 7 & $2 s_{D-1}$ & $\Lambda\left((2 N) \sigma_{D-1}^{ \pm}\right)$ \\
\hline
\end{tabular}

We now extend these results to the case when $p$ has zero mass. Let $V=\mathbb{R}^{1, D-1}(D \geq 4)$ and $p \neq 0$ a null vector in $V$. Let $e_{j}$ be the standard basis vectors for $V$ so that $\left(e_{0}, e_{0}\right)=-\left(e_{j}, e_{j}\right)=1$ for $j=1, \ldots, D-1$. We may assume that $p=e_{0}+e_{1}$. Let $V_{1}^{\prime}$ be the span of $e_{j}(2 \leq j \leq D-1)$. The signature of $V_{1}^{\prime}$ is $(0, D-2)$. Then we have the flag $0 \subset \mathbb{R} p \subset p^{\perp} \subset V$ left stable by the stabilizer $L_{0}^{p}$ of $p$ in $L_{0}$. The quadratic form on $p^{\perp}$ has $\mathbb{R} p$ as its radical and so induces a nondegenerate form on $V_{1}:=p^{\perp} / \mathbb{R} p$. Write $L_{1}=\operatorname{Spin}\left(V_{1}\right)$. Note that $V_{1}^{\prime} \simeq V_{1}$.

We have a map $x \mapsto x^{\prime}$ from $L_{0}^{p}$ to $L_{1}$ where, for $v \in p^{\perp}$ with image $v^{\prime} \in V_{1}$, $x^{\prime} v^{\prime}=(x v)^{\prime}$. It is known that this is surjective and its kernel $T_{1}:=T_{0}^{p}$ is isomorphic to $V_{1}$ canonically: for $x \in T_{0}^{p}$, the vector $x e_{0}-e_{0} \in p^{\perp}$, and the map that sends $x$ to the image $t(x)$ of $x e_{0}-e_{0}$ in $V_{1}$ is well defined and is an isomorphism of $T_{0}^{p}$ with $V_{1}$. The map $x \mapsto\left(t(x), x^{\prime}\right)$ is an isomorphism of $L_{0}^{p}$ with the semidirect product $V_{1} \times L_{1}$. The Lie algebra of the big spin group $L_{0}$ has the $e_{r} e_{s}(r<s)$ as basis and it is a simple calculation that the Lie algebra of $L_{0}^{p}$ has as basis $t_{j}=\left(e_{0}+e_{1}\right) e_{j}(2 \leq j \leq D-1), e_{r} e_{s}(2 \leq r<s \leq D-1)$ with the $t_{j}$ forming a basis of the Lie algebra of $T_{1}$. The $e_{r} e_{s}(2 \leq r<s \leq D-1)$ span a Lie subalgebra of the Lie algebra of $L_{0}$ and the corresponding subgroup $H \subset L_{0}^{p}$ is such that $L_{0}^{p} \simeq T_{1} \times^{\prime} H$. For all of this see $\operatorname{Var} 04$, pp. 36-37.

We shall now determine the structure of the restriction to $L_{0}^{p}$ of the irreducible spin representation(s) of $L$, over $\mathbb{R}$ as well as over $\mathbb{C}$. Since this may not be known widely we give some details. We begin with some preliminary remarks.

Let $U$ be any finite dimensional complex $L_{0}^{p}$-module. Write $U=\oplus_{\chi} U_{\chi}$ where $U_{\chi}$, for any character (not necessarily unitary) $\chi$ of $T_{1}$, is the subspace of all elements $u \in U$ such that $(t-\chi(t))^{m} u=0$ for sufficiently large $m$. The action of $L_{1}$ permutes the $U_{\chi}$, and so, since $L_{1}$ has no finite nontrivial 
orbit in the space of characters of $T_{1}$, it follows that the spectrum of $T_{1}$ consists only of the trivial character, i.e., $T_{1}$ acts unipotently. In particular $U_{1}$, the subspace of $T_{1}$-invariant elements of $U$, is $\neq 0$, an assertion which is then valid for real modules also. It follows that we have a strictly increasing filtration $\left(U_{i}\right)_{i \geq 1}$ where $U_{i+1}$ is the preimage in $U$ of $\left(U / U_{i}\right)_{1}$. In particular, if $U$ is semisimple, $U=U_{1}$.

Lemma 21 Let $W$ be an irreducible real or complex spin module for $L_{0}$. Let $W_{1}$ be the subspace of all elements of $W$ fixed by $T_{1}$ and $W^{1}:=W / W_{1}$. We then have the following.

(i) $0 \neq W_{1} \neq W, W_{1}$ is the unique proper non zero $L_{0}^{p}$-submodule of $W$, and $T_{1}$ acts trivially also on $W^{1}$.

(ii) $W_{1}$ and $W^{1}$ are both irreducible $L_{0}^{p}$-modules on which $L_{0}^{p} / T_{1} \simeq L_{1}$ acts as a spin module.

(iii) The exact sequence

$$
0 \longrightarrow W_{1} \longrightarrow W \longrightarrow W / W_{1} \longrightarrow 0
$$

does not split.

(iv) Over $\mathbb{R}, W, W_{1}, W^{1}$ are all of the same type. If $\operatorname{dim}(V)$ is odd, $W_{1} \simeq$ $W^{1}$. Let $\operatorname{dim}(V)$ be even; then, over $\mathbb{C}, W_{1}, W^{1}$ are the two irreducible spin modules for $L_{1}$; over $\mathbb{R}, W_{1} \simeq W^{1}$ when $W$ is of complex type, namely, when $D \equiv 0,4 \bmod 8$; otherwise, the modules $W_{1}, W^{1}$ are the two irreducible modules of $L_{1}$ (which are either real or quaternionic).

Proof. We first work over $\mathbb{C}$. Let $C$ be the Clifford algebra of $V$. The key point is that $W_{1} \neq W$. Suppose $W_{1}=W$. Then $t_{j}=0$ on $W$ for all $j$. If $D$ is odd, $C^{+}$is a full matrix algebra and so all of its modules are faithful, giving a contradiction. Let $D$ be even and $W$ one of the spin modules for $L_{0}$. We know that inner automorphism by the invertible odd element $e_{2}$ changes $W$ to the other spin module. But as $e_{2} t_{j} e_{2}^{-1}=t_{j}$ for $j>2$ and $-t_{2}$ for $j=2$, it follows that $t_{j}=0$ on the other spin module also. Hence $t_{j}=0$ in the irreducible module for the full Clifford algebra $C$. Now $C$ is isomorphic to a full matrix algebra and so its modules are faithful, giving again $t_{j} \neq 0$, a contradiction.

Let $\left(W_{i}\right)$ be the strictly increasing flag of $L_{0}^{p}$-modules, with $T_{1}$ acting trivially on each $W_{i+1} / W_{i}$, defined by the previous discussion. Let $m$ be such that $W_{m}=W$. Clearly $m \geq 2$. On the other hand, the element -1 of $L_{0}$ lies in $L_{0}^{p}$ and as it acts as -1 on $W$, it acts as -1 on all the $W_{i+1} / W_{i}$. 
Hence $\operatorname{dim}\left(W_{i+1} / W_{i}\right) \geq \operatorname{dim}\left(\sigma_{D-2}\right)$ (see Lemma 6.8.1 of [Var04]), and there is equality if and only if $W_{i+1} / W_{i} \simeq \sigma_{D-2}$. Since $\operatorname{dim}\left(\sigma_{D}\right)=2 \operatorname{dim}\left(\sigma_{D-2}\right)$, we see at once that $m=2$ and that both $W_{1}$ and $W / W_{1}$ are irreducible $L_{0^{-}}^{p}$ modules which are spin modules for $L_{1}$. The exact sequence in (iii) cannot split, as otherwise $T_{1}$ will be trivial on all of $W$. Suppose now $U$ is a non zero proper $L_{0}^{p}$-submodule of $W$. Then $\operatorname{dim}(U)=\operatorname{dim}\left(W_{1}\right)$ for the same dimensional argument as above, and so $U$ is irreducible, thus $T_{1}$ is trivial on it, showing that $U=W_{1}$. We have thus proved (i)-(iii).

We now prove (iv). There is nothing to prove when $D$ is odd since there is only one spin module. Suppose $D$ is even. Let us again write $\sigma_{D-2}^{ \pm}$for the $L_{0}^{p}$-modules obtained by lifting the irreducible spin modules of $L_{1}$ to $L_{0}^{p}$. We consider two cases.

$D \equiv 0,4 \bmod 8:$ In this case $\sigma_{D}^{ \pm}$are self dual while $\sigma_{D-2}^{ \pm}$are dual to each other. It is not restrictive to assume $W=\sigma_{D}^{+}$and $W_{1}=\sigma_{D-2}^{+}$. We have the quotient map $W=\sigma_{D}^{+} \longrightarrow W^{1}=\sigma$; and $\sigma$ is to be determined. Writing $\sigma^{\prime}$ for the dual of $\sigma$, we get $\sigma^{\prime} \subset\left(\sigma_{D}^{+}\right)^{\prime} \simeq \sigma_{D}^{+}$, so that, by the uniqueness of the submodule proved above, $\sigma^{\prime}=\sigma_{D-2}^{+}$. Hence $\sigma=\sigma_{D-2}^{-}$.

$D \equiv 2,6 \bmod 8:$ Now $\sigma_{D}^{ \pm}$are dual to each other while $\sigma_{D-2}^{ \pm}$are self dual. Again, suppose $W=\sigma_{D}^{+}$and $W_{1}=\sigma_{D-2}^{+}$. The above argument then gives $\sigma^{\prime} \subset \sigma_{D}^{-}$. On the other hand, the inner automorphism by $e_{2}$ transforms $\sigma_{D}^{+}$ into $\sigma_{D}^{-}$, and the subspace of $\sigma_{D}^{+}$fixed by $T_{1}$ into the corresponding subspace of $\sigma_{D}^{-}$, while at the same time changing $\sigma_{D-2}^{+}$into $\sigma_{D-2}^{-}$. Hence it changes the inclusion $\sigma_{D-2}^{+} \subset \sigma_{D}^{+}$into the inclusion $\sigma_{D-2}^{-} \subset \sigma_{D}^{-}$. Hence we have $\sigma^{\prime}=\sigma_{D-2}^{-}$. Dualizing, this gives $\sigma=\sigma_{D-2}^{-}$once again. This finishes the proof of the lemma over $\mathbb{C}$.

We now work over $\mathbb{R}$. Since both $V$ and $V_{1}$ have the same signature $D-2$, it is immediate that the real spin modules for $L_{0}$ and $L_{1}$ are of the same type. As an $L_{0}$-module, the complexification $W_{\mathbb{C}}$ of $W$ is either irreducible or is a direct sum $U \oplus \bar{U}$ where $U$ is a complex spin module for $L_{0}$. In the first case $W$ is of real type and the lemma follows from the lemma for the complex spin modules. In the second case $W$ is of quaternionic or complex type according as $U$ and $\bar{U}$ are equivalent or not.

Complex type: We have $W_{\mathbb{C}}=U \oplus Z$ where $Z=\bar{U}$ is the complex conjugate of $U$. We have $U \simeq \sigma_{D}^{+}, Z \simeq \sigma_{D}^{-}$. Since $\mathbb{C} W_{1}=U_{1} \oplus Z_{1}$ it is clear that $0 \neq W_{1} \neq W$. The real irreducible spin modules of $L_{1}$ have dimension $2^{D / 2-1}$ and so we find that $\operatorname{dim}\left(W_{1}\right)=2^{D / 2-1}$ and $W_{1}, W^{1}$ are both irreducible; they are equivalent as they are of complex type. A non zero proper submodule $R$ of $W$ then has dimension $2^{D / 2-1}$ and so must be irreducible. Hence either $R=W_{1}$ or $W=W_{1} \oplus R$. But then $W=W_{1}$, 
a contradiction. The same argument shows that the exact sequence in the lemma does not split.

Quaternionic type: For proving (i)-(iii) of the lemma the argument is the same as in the complex type, except that $Z \simeq U$. We now check (iv). The case of odd dimension is obvious. So let $D$ be even and $W_{1} \simeq s_{D-2}^{+}$. Then $\mathbb{C} W_{1} \simeq 2 \sigma_{D-2}^{+}$so that $U_{1} \simeq Z_{1} \simeq \sigma_{D-2}^{+}$. Then $U^{1} \simeq Z^{1} \simeq \sigma_{D-2}^{-}$and so $\mathbb{C} W^{1} \simeq 2 \sigma_{D-2}^{-}$. Hence $W^{1} \simeq s_{D-2}^{-}$.

The lemma is completely proved.

Remark 7 Since we are interested in the quotient $W^{1}$ rather than $W_{1}$ below, we change our convention slightly; for $W=s_{D}^{ \pm}$we write $W^{1}=s_{D-2}^{ \pm}$and $W_{1}=s_{D-2}^{\mp}$.

We now come to the discussion of the structure of $\kappa_{p}$ when $p$ is in a massless orbit.

Proposition 9 Let $p_{0}>0,\langle p, p\rangle=0$. Then $\operatorname{rad}\left(\Phi_{p}\right)=\mathfrak{g}_{1}^{T_{1}}$, the subspace of elements of $\mathfrak{g}_{1}$ fixed by $T_{1}$. Moreover $T_{1}$ acts trivially on $\mathfrak{g}_{1 p}, \operatorname{rad}\left(\Phi_{p}\right) \simeq \mathfrak{g}_{1 p}$ except in the cases $D \equiv 2,6 \bmod 8$ when $\operatorname{rad}\left(\Phi_{p}\right)$ and $\mathfrak{g}_{1 p}$ are dual to each other, and $L_{0}^{p} / T_{1} \simeq L_{1}$ acts spinorially on $\mathrm{rad} \Phi_{p}$ and $\mathfrak{g}_{1 p}$. In all cases $\operatorname{dim}\left(\operatorname{rad}\left(\Phi_{p}\right)\right)=\operatorname{dim}\left(\mathfrak{g}_{1 p}\right)=(1 / 2) \operatorname{dim}\left(\mathfrak{g}_{1}\right)$. For $\mathfrak{g}_{1 p}$ as well as the associated $\kappa_{p}$ the results are as in the following table.

$$
\begin{aligned}
& D \bmod 8 \quad \mathfrak{g}_{1 p} \quad \kappa_{p} \\
& 0,4 \quad N s_{D-2} \quad \Lambda\left(N \sigma_{D-2}^{ \pm}\right) \\
& 2\left(N^{ \pm}=2 n^{ \pm}\right) \quad N^{+} s_{D-2}^{+}+N^{-} s_{D-2}^{-} \quad \Lambda\left(n^{+} \sigma_{D-2}^{+}+n^{-} \sigma_{D-2}^{-}\right) \\
& 6 \quad N^{+} s_{D-2}^{+}+N^{-} s_{D-2}^{-} \quad \Lambda\left(N^{+} \sigma_{D-2}^{+}+N^{-} \sigma_{D-2}^{-}\right) \\
& 1,3(N=2 n) \quad N s_{D-2} \quad \Lambda\left(n \sigma_{D-2}\right) \\
& 5,7 \quad N s_{D-2} \quad \Lambda\left(N \sigma_{D-2}\right)
\end{aligned}
$$

Proof. We have $\mathfrak{g}_{1}=\oplus_{1 \leq i \leq N} \mathfrak{h}_{i}$ where the $\mathfrak{h}_{i}$ are real irreducible spin modules and $\left[\mathfrak{h}_{i}, \mathfrak{h}_{j}\right]=0$ for $i \neq j$ while $\langle[X, X], q\rangle>0$ for all $q \in \Gamma^{+}$. Let $\mathfrak{r}_{p}=\operatorname{rad} \Phi_{p}$ and $\mathfrak{r}_{i p}$ the radical of the restriction of $\Phi_{p}$ to $\mathfrak{h}_{i}$. Since $Q_{p}(X)=\sum_{i} Q_{p}\left(X_{i}\right)$ where $X_{i}$ is the component of $X$ in $\mathfrak{h}_{i}$, it follows that $\mathfrak{r}_{p}=\oplus_{i} \mathfrak{r}_{i p}$. We now claim that $\mathfrak{r}_{i p}=\left(\mathfrak{h}_{i}\right)_{1}$, namely, the subspace of elements of $\mathfrak{h}_{i}$ fixed by $T_{1}$. Since $\mathfrak{r}_{i p}$ is a $L_{0}^{p}$-submodule it suffices, in view of the lemma above, to show that $0 \neq \mathfrak{r}_{i p} \neq \mathfrak{h}_{i}$. If $\mathfrak{r}_{i p}$ were $0, \Phi_{p}$ would be strictly positive definite on $\mathfrak{h}_{i}$, and hence the action of $L_{0}^{p}$ will have an invariant positive definite quadratic form. So the action of $L_{0}^{p}$ on $\mathfrak{h}_{i}$ will be semisimple, implying that $T_{1}$ will act trivially on $\mathfrak{h}_{i}$. This is impossible, since, by the preceding lemma, $\mathfrak{h}_{i} \neq\left(\mathfrak{h}_{i}\right)_{1}$. If $\mathfrak{r}_{i p}=\mathfrak{h}_{i}$, then $\Phi_{p}=0$ on $\mathfrak{h}_{i}$, and this will imply that $p=0$. Thus $\mathfrak{r}_{i p}=\left(\mathfrak{h}_{i}\right)_{1}$, 
hence $\mathfrak{r}_{p}=\left(\mathfrak{g}_{1}\right)_{1}$. The other assertions except the table are now clear. For the table we need to observe that $\mathfrak{g}_{1 p}=\oplus_{i} \mathfrak{h}_{i} / \mathfrak{r}_{i p}$ and that $\mathfrak{r}_{i p} \simeq \mathfrak{h}_{i} / \mathfrak{r}_{i p}$ except when $D \equiv 2,6 \bmod 8$; in these cases, the two modules are the two real or quaternionic spin modules which are dual to each other. The table is worked out in a similar manner to Proposition 8 . We omit the details.

Remark 8 The result that the dimension of $\mathfrak{g}_{1 p}$ has $1 / 2$ the dimension of $\mathfrak{g}_{1}$ extends the known calculations when $D=4$ (see [FSZ81]).

\subsubsection{The role of the $R$-group in classifying the states of $\kappa_{p}$}

In the case of $N$-extended supersymmetry we have two groups acting on $\mathfrak{g}_{1}$ : $L_{0}^{p}$, the even part of the little super group at $p$, and the $R$-group ([Del99], Var04]) $R$. Their actions commute and they both leave the quadratic form $Q_{p}$ invariant. In the massive case we have a map

$$
L_{0}^{p} \times R \longrightarrow \operatorname{Spin}\left(\mathfrak{g}_{1 p}\right)
$$

so that one can speak of the restriction $\kappa_{p}^{\prime}$ of the spin representation of $\operatorname{Spin}\left(\mathfrak{g}_{1 p}\right)$ to $L_{0}^{p} \times R$. The same is true in the massless case except we have to replace $L_{0}^{p}$ by a two-fold cover of it. It is thus desirable to not just determine $\kappa_{p}$ as we have done but actually determine this representation $\kappa_{p}^{\prime}$ of $L_{0}^{p} \times R$. We have not done this but there is no difficulty in principle. However, when $D=4$, we have a beautiful formula FSZ81. To describe this, assume that we are in the massive case. We first remark that $\mathfrak{g}_{1} \simeq \mathbb{H}^{N} \otimes_{\mathbb{H}} S_{0}$ where $S_{0}$ is the quaternionic irreducible of $\mathrm{SU}(2)$ of dimension 4 . Thus the $R$-group is the unitary group $\mathrm{U}(N, \mathbb{H})$. Over $\mathbb{C}$ we thus have $\mathfrak{g}_{1 \mathbb{C}} \simeq \mathbb{C}^{2 N} \otimes \mathbb{C}^{2}$ where the $R$-group is the symplectic group $\operatorname{Sp}(2 N, \mathbb{C})$ acting on the first factor and $L_{0}^{p} \simeq$ $\mathrm{SU}(2)$ acts as $D^{1 / 2}$ on the second factor. The irreducible representations of $\mathrm{Sp}(2 N, \mathbb{C}) \times \mathrm{SU}(2)$ are outer tensor products of irreducibles $a$ of the first factor and $b$ of the second factor, written as $(a, b)$. Let $\mathbf{k}$ denote the irreducible of dimension $k$ of $\mathrm{SU}(2)$ and $[2 N]_{k}$ denote the irreducible representation of the symplectic group in the space of traceless antisymmetric tensors of rank $k$ over $\mathbb{C}^{2 N}$; by convention for $k=0$ this is the trivial representation and for $k=1$ it is the vector representation. Then

$$
\kappa_{p}^{\prime} \simeq\left([2 N]_{0}, \mathbf{N}+\mathbf{1}\right)+\left([2 N]_{1}, \mathbf{N}\right)+\ldots\left([2 N]_{k}, \mathbf{N}+\mathbf{1}-\mathbf{k}\right)+\ldots\left([2 N]_{N}, \mathbf{1}\right) .
$$

To see how this follows from our theory note that $\mathbb{C}^{2 N} \otimes e_{1}$ is a subspace satisfying the conditions of Lemma 19] for the symplectic group and so $\kappa_{p} \simeq$ $\Lambda\left(\mathbb{C}^{2 N}\right)$. It is known that

$$
\Lambda\left(\mathbb{C}^{2 N}\right) \simeq \sum_{0 \leq k \leq N}(N+1-k)[2 N]_{k} .
$$


On the other hand we know that the representations of $\mathrm{SU}(2)$ in $\kappa_{p}$ are precisely the $\mathbf{N}+\mathbf{1}-\mathbf{k}(0 \leq k \leq N)$. The formula for $\kappa_{p}^{\prime}$ is now immediate. In the massless case the $R$-group becomes $\mathrm{U}(N)$ and

$$
\kappa_{p}^{\prime} \simeq \sum_{0 \leq k \leq N}\left((N-k) / 2,[N]_{k}\right)
$$

where $r / 2$ denotes the character denoted earlier by $\chi_{r}$ and $[N]_{k}$ is the irreducible representation of $\mathrm{U}(N)$ defined on the space $\Lambda^{k}\left(\mathbb{C}^{N}\right)$. We omit the proof which is similar.

Acknowledgement.G. C. gratefully acknowledges a grant of the Università di Genova that has made possible a visit to Los Angeles during which some of the work for this paper has been done. He thanks Professor V. S. Varadarajan for his warm hospitality.

V. S. V. would like to thank professor Giuseppe Marmo and INFN, Naples, professor Sergio Ferrara and CERN, Geneva, and Professors Enrico Beltrametti and Gianni Cassinelli and INFN, Genoa, for their hospitality during the summers of 2003 and 2004, during which most of the work for this paper was done. We are grateful to Professor Pierre Deligne of the Institute for Advanced Study, Princeton, NJ, for his interest in our work and for his comments which have improved the paper.

\section{References}

[Del99] Pierre Deligne. Notes on spinors. In Quantum fields and strings: a course for mathematicians, Vol. 1, 2 (Princeton, NJ, 1996/1997), pages 99-135. Amer. Math. Soc., Providence, RI, 1999.

[DM78] Jacques Dixmier and Paul Malliavin. Factorisations de fonctions et de vecteurs indéfiniment différentiables. Bull. Sci. Math. (2), 102(4):307$330,1978$.

[DM99] Pierre Deligne and John W. Morgan. Notes on supersymmetry (following Joseph Bernstein). In Quantum fields and strings: a course for mathematicians, Vol. 1, 2 (Princeton, NJ, 1996/1997), pages 41-97. Amer. Math. Soc., Providence, RI, 1999.

[DP85] V. K. Dobrev and V. B. Petkova. All positive energy unitary irreducible representations of extended conformal supersymmetry. Phys. Lett. B, 162(1-3):127-132, 1985. 
[DP86] V. K. Dobrev and V. B. Petkova. All positive energy unitary irreducible representations of the extended conformal superalgebra. In Conformal groups and related symmetries: physical results and mathematical background (Clausthal-Zellerfeld, 1985), volume 261 of Lecture Notes in Phys., pages 300-308. Springer, Berlin, 1986.

[DP87] V. K. Dobrev and V. B. Petkova. Group-theoretical approach to extended conformal supersymmetry: function space realization and invariant differential operators. Fortschr. Phys., 35(7):537-572, 1987.

[Fer01] Sergio Ferrara. UCLA lectures on supersymmetry. 2001.

[Fer03] Sergio Ferrara. UCLA lectures on supersymmetry. 2003.

[FSZ81] S. Ferrara, C. A. Savoy, and B. Zumino. General massive multiplets in extended supersymmetry. Phys. Lett. B, 100(5):393-398, 1981.

[Nel59] Edward Nelson. Analytic vectors. Ann. of Math. (2), 70:572-615, 1959.

[SS74] Abdus Salam and J. Strathdee. Unitary representations of supergauge symmetries. Nuclear Phys., B80:499-505, 1974.

[Var04] V. S. Varadarajan. Supersymmetry for mathematicians: an introduction, volume 11 of Courant Lecture Notes in Mathematics. New York University Courant Institute of Mathematical Sciences, New York, 2004.

[Wit82] Edward Witten. Supersymmetry and Morse theory. J. Differential Geom., 17(4):661-692 (1983), 1982. 